\title{
Krull dimension for limit groups
}

\author{
LARSEN LOUDER
}

\begin{abstract}
We show that varieties defined over free groups have finite Krull dimension, answering a question of Z Sela.
\end{abstract}

20F65; 20E05, 20E06

\section{Introduction}

The purpose of this paper is to provide a dimension theory for varieties defined over free groups. Given a variety $V$ defined over a field, chains of proper inclusions of irreducible subvarieties have length bounded above by some smallest number, the dimension of $V$. Solutions to systems of equations defined over a free group $\mathbb{F}$ may also be decomposed into irreducible components; see Sela [14], Baumslag, Myasnikov and Remeslennikov [2] and Kharlampovich and Myasnikov [9]. Each irreducible component may be identified with the set of homomorphisms from a limit group to a free group, and a chain of irreducible subvarieties corresponds to a sequence of epimorphisms of limit groups. We define the Krull dimension of a variety $V$ defined over a nonabelian free group to be the supremum of lengths of chains of irreducible subvarieties contained in $V$.

Theorem 1.1 (Krull dimension) There is a function $D(N)$ such that if

$$
\mathbb{F}_{N} \rightarrow L_{1} \rightarrow \cdots \rightarrow L_{k}
$$

is a sequence of proper epimorphisms of limit groups, then $k \leq D(N)$.

Given a sequence of epimorphisms of limit groups we construct uniformly many sequences of strictly lower complexity, bound together in a resolution, which we can arrange to be a graph of sequences of groups, modeled on a common Grushko or JSJ decomposition. Using our results from [10] we lift a dimension bound for the (simpler) derived sequences to a bound on the length of the resolution, and from this to a bound on the length of original sequence. 
Acknowledgements I am deeply indebted to my advisor, Mladen Bestvina, and Mark Feighn. I would also like to extend many thanks to Matt Clay, Chloé Perin, Zlil Sela and the anonymous referee, whose remarks have really helped improve the paper.

I would like to acknowledge support from the University of Utah, MSRI, RutgersNewark, the National Science Foundation, The University of Michigan and The University of Oxford.

\section{Basic facts}

In this section we establish notation and review basic facts from the theory. A nonabelian free group is usually denoted by $\mathbb{F}$. The centralizer of $E<G$ is denoted by $Z_{G}(E)$. Let $V$ be a vertex group of a graph of groups decomposition. The set of images in $V$ of edge groups incident to $V$ is denoted by $\mathcal{E}(V)$. If $\Delta$ is a graph of groups decomposition then $T_{\Delta}$ is the Bass-Serre tree corresponding to $\Delta$. A group is CSA if maximal abelian subgroups are malnormal. A homomorphism is proper if it has nontrivial kernel.

The phrase " $X$ is controlled by $Y$ " should be read as "there is a function $f$ such that $X \leq f(Y)$ ", for instance, "rank is controlled by first betti number and depth."

A closed subset of a topological space is irreducible if it is not the union of finitely many proper closed subsets.

Definition 2.1 (Limit group) Let $G$ be a finitely generated group. A sequence $f_{n} \in \operatorname{Hom}(G, \mathbb{F})$ such that for all $g \in G$, either $f_{n}(g)=1$ for sufficiently large $n$, or $f_{n}(g) \neq 1$ for sufficiently large $n$, is stable. The stable kernel of a stable sequence $f_{n}$ is the normal subgroup of $G$ generated by all elements which have trivial image for sufficiently large $n$, and is denoted by $\underset{\operatorname{Ker}}{\longrightarrow}\left(f_{n}\right)$. A quotient of a finitely generated group by the stable kernel of a stable sequence is a limit group.

A stable sequence $f_{n}$ converges to $G / \underset{\operatorname{Ker}}{\longrightarrow}\left(f_{n}\right)$ in the sense that the sequence $\operatorname{ker}\left(f_{n}\right)$ converges to $\underset{\operatorname{Ker}}{\longrightarrow}\left(f_{n}\right)$ in the space of normal subgroups of $G$. Limit groups are the finitely generated groups in the closure of the marked free groups. One simple consequence of this picture is that nonabelian free groups are precisely the groups with the same universal theory as nonabelian free groups. See Champetier and Guirardel [5] for a treatment from this point of view.

A finitely generated group $L$ is fully residually free if, for every finite subset $S \subset L$ there is a homomorphism $f: L \rightarrow \mathbb{F}$ embedding $S$. Limit groups are fully residually free by Sela [14, Theorem 4.6], and fully residually free groups are clearly limit groups. 
Consider the space $\mathbb{F}^{n}$, which we identify with $\operatorname{Hom}\left(\left\langle x_{1}, \ldots, x_{n}\right\rangle, \mathbb{F}\right)$. We give $\mathbb{F}^{n}$ the Zariski topology, generated by closed sets of the form

$$
\left\{\left(x_{1}, \ldots, x_{n}\right) \mid w\left(x_{i}\right)=1\right\},
$$

where $w$ is some word in $x_{i}$. Every closed set is the union of finitely many closed sets of the form $\operatorname{Hom}(G, \mathbb{F})$, where $G$ is a residually free quotient of $\left\langle x_{i}\right\rangle$. If $\operatorname{Hom}(G, \mathbb{F})$ is irreducible, then, for every $g_{1}, \ldots, g_{m} \in G \backslash\{1\}$,

$$
\operatorname{Hom}(G, \mathbb{F}) \not \subset \bigcup_{j=1, \ldots, m} \operatorname{Hom}\left(G /\left\langle g_{j}\right\rangle, \mathbb{F}\right),
$$

which is precisely what it means for $G$ to be fully residually free.

\subsection{Structure theory/JSJ decompositions}

Our main tool is the JSJ decomposition. See Bestvina and Feighn [4] and Sela [14] for the case of a limit group and Dunwoody and Sageev [7], Rips and Sela [11] and Fujiwara and Papasoglu [8] for a more general theory.

Definition 2.2 (Generalized abelian decomposition [4; 14]) A GAD, or generalized abelian decomposition, or, for short, an abelian decomposition, of a freely indecomposable finitely generated group $G$, is a graph of groups $\Delta\left(R_{i}, A_{j}, Q_{k}, E_{l}\right)$ of $G$ over vertex groups $R_{i}, A_{j}, Q_{k}$, and abelian edge groups $E_{l}$ such that:

- $A_{j}$ is abelian.

- $Q_{k}$ is the fundamental group of a compact surface with boundary $\Sigma_{k}$, such that the Euler characteristic of $\Sigma_{k}$ is at most -2 or $\Sigma_{k}$ is a torus with one boundary component. In Rips/Sela's terminology the $Q_{k}$ are "quadratically hanging," or QH for short.

- Any edge group incident to a $\mathrm{QH}$ vertex group is conjugate into a subgroup corresponding to a boundary component.

The $R_{i}$ are called rigid. Let $\mathcal{E}$ be a collection of subgroups of $G$. A GAD relative to $\mathcal{E}$ is a GAD of $G$ in which every element of $\mathcal{E}$ is elliptic (conjugate into a vertex group, or equivalently, fixing a point in $T_{\Delta}$ ). A cyclic decomposition is a GAD over infinite cyclic edge groups.

The peripheral subgroup [4] $P(A)$ of an abelian vertex group $A$ of a graph of groups decomposition is the intersection of all kernels of maps $A \rightarrow \mathbb{Z}$ killing all images of incident edge groups, and in the case of a GAD relative to $\mathcal{E}$, the peripheral 
subgroup $P(A)$ of $A$ is the intersection of all kernels of maps $A \rightarrow \mathbb{Z}$ killing all images of incident edge groups and conjugates of elements of $\mathcal{E}$ contained in $A$. The peripheral subgroup is the smallest direct summand of $A$ containing the images of all edge groups incident to $A$, and, in the relative case, conjugates of elements of $\mathcal{E}$ contained in $A$ as well.

Two GADs of a limit group are equivalent if they have the same elliptic subgroups. Equivalent GADs can be obtained from each other by sequences of folds [11]. A splitting is visible in a GAD if it corresponds to cutting a $\mathrm{QH}$ vertex group along a simple closed curve, a splitting of an abelian vertex group in which the peripheral subgroup is elliptic, or is a one-edged splitting visible in an equivalent GAD.

If $\Delta$ is a GAD, then we say that $H<G$ is $\Delta$-elliptic if it elliptic in every one-edged splitting of $G$ visible in $\Delta$. Let $\operatorname{Ell}(\Delta)$ be the set of $\Delta$-elliptic subgroups; Ell( $\Delta)$ consists of the rigid vertex groups, peripheral subgroups of abelian vertex groups, their subgroups, and all their conjugates.

A splitting of a group is admissible if edge groups are abelian and noncyclic abelian subgroups are elliptic. An admissible one edged splitting is root-closed if the edge group is closed under taking roots. Let $G$ be a freely indecomposable (relative to $\mathcal{E}$ ) finitely generated group, and let $\mathcal{C}$ be a finite family of admissible one-edged splittings of $G($ rel $\mathcal{E})$. The main construction of JSJ theory is that given such a family of splittings $\mathcal{C}$ there is a GAD $\Delta_{\mathcal{C}}$ such that $\operatorname{Ell}\left(\Delta_{\mathcal{C}}\right)=\cap_{C \in \mathcal{C}} \operatorname{Ell}(C)$.

An abelian JSJ of a freely indecomposable limit group $G$ is a GAD corresponding to the collection of all admissible, root-closed, one-edged splittings, and is denoted $\mathrm{JSJ}(G)$. For each finite family $\mathcal{C}$ of splittings there is an associated GAD and existence of the abelian JSJ of a limit group is ensured by a uniform bound on the size of the decompositions $\Delta_{\mathcal{C}}[14$, Theorem 3.9].

Suppose that $(G, \mathcal{E})$ is freely indecomposable. The abelian JSJ of $G$ relative to $\mathcal{E}$, denoted $\operatorname{JSJ}(G, \mathcal{E})$, is a JSJ decomposition associated to the family of all root-closed abelian splittings of $G$ in which all elements of $\mathcal{E}$ are elliptic.

A limit group $G$ is elementary if it is free, free abelian, or the fundamental group of a closed surface. If $G$ is nonelementary and freely indecomposable then it has a nontrivial abelian JSJ [14, Theorem 3.2].

Definition 2.3 [14] A one-edged splitting over a cyclic subgroup is inessential if at least one vertex group is cyclic, and is essential otherwise. A principal cyclic splitting of a limit group is an essential one-edged splitting $G \cong A *_{C} B$ or $G \cong A *_{C}$, over a cyclic subgroup $C$, such that either $Z_{G}(C)$ is cyclic or $A$ is abelian. 
A principal cyclic decomposition is a GAD in which every visible splitting is either over a boundary subgroup of a $\mathrm{QH}$ vertex group, or such that there is a principal cyclic splitting over a subgroup of the centralizer of the edge group. The principal cyclic JSJ of a freely indecomposable limit group $L$ is the JSJ decomposition $\operatorname{PJSJ}(L)$ corresponding to the family of root-closed principal cyclic splittings. We denote the relative principal cyclic $\mathrm{JSJ}$ by $\operatorname{PJSJ}(L, \mathcal{E})$. The principal cyclic JSJ is a principal cyclic decomposition.

The restricted principal cyclic JSJ, $\operatorname{RJSJ}(G)$, or restricted JSJ for short, of a freely indecomposable limit group $G$ with $\mathrm{QH}$ subgroups is the JSJ decomposition associated to the set of principal cyclic splittings whose edge groups are hyperbolic in some other principal cyclic splitting, and is obtained from the abelian JSJ by collapsing all edges not adjacent to $\mathrm{QH}$ vertex groups. If $G$ has no $\mathrm{QH}$ vertex groups, then we define the restricted JSJ to be the principal cyclic JSJ.

Since limit groups have principal cyclic splittings [14, Theorem 3.2], the principal cyclic JSJ of a freely indecomposable nonelementary limit group is nontrivial. Splittings visible in the principal cyclic JSJ might not be principal: For instance, a boundary component of a $\mathrm{QH}$ vertex group may be the only edge group attached to a cyclic vertex group, or may be centralized by a noncyclic subgroup of a rigid vertex group of the abelian JSJ. In the first case the splitting corresponding to the boundary component is not essential, and in the second case the splitting is not principal because the centralizer is noncyclic, but isn't an abelian vertex group of the JSJ with cyclic peripheral subgroup.

Definition 2.4 (Neighborhood) The neighborhood $\mathcal{N}(V)$ of a vertex group $V$ of a GAD is the subgroup generated by $V$ and conjugates of non-QH adjacent vertex groups which intersect $V$ nontrivially.

A more explicit description of the principal cyclic JSJ is as follows: Consider the abelian JSJ of a freely indecomposable limit group $G$. All QH vertex groups of JSJ $(G)$ appear as vertex groups of $\operatorname{PJSJ}(G)$. If $A$ is an abelian vertex group of $\operatorname{JSJ}(G)$ with noncyclic peripheral subgroup there is no principal cyclic splitting of $G$ over a subgroup of $A$, and the neighborhood of $A$ must be elliptic in $\operatorname{PJSJ}(G)$. If $R$ is a rigid vertex group of $G$ and an edge group $E$ incident to $R$ has noncyclic centralizer in $R$, then the neighborhood of the abelian vertex group adjacent to $E$ is also elliptic in $\operatorname{PJSJ}(G)$.

Note that the requirement that the splittings used in the construction of the abelian JSJ be root-closed implies that edge groups are usually closed under taking roots. Edges not adjacent to $\mathrm{QH}$ vertex groups are closed under taking roots, but edges connected to $\mathrm{QH}$ vertex groups might not be. If $e$ is incident to a $\mathrm{QH}$ vertex group $Q$ then the 
map $G_{e} \hookrightarrow Q$ is an isomorphism with a boundary subgroup of $Q$. Furthermore, if $A$ is a valence one vertex group and the incident edge group has finite index in $A$ then the edge is attached to a $\mathrm{QH}$ vertex group and the index of the edge in $A$ is at least three, otherwise a $\mathrm{QH}$ vertex group could be enlarged. Rigid vertex groups are always closed under taking roots.

JSJ decompositions are unique only up to folds (see Section 5.2) preserving elliptic subgroups. Some abelian decompositions are more convenient to work with and we assume throughout that the JSJ of a limit group is 2-acylindrical, that noncyclic abelian subgroups are elliptic, and, by folding, that each edge is adjacent to a unique maximal abelian vertex group. ${ }^{1}$

Let $(L, \mathcal{E})$ be a limit group and let $\operatorname{JSJ}(L, \mathcal{E})$ be its relative abelian JSJ. Without loss we may assume the following normalizations hold for $\operatorname{JSJ}(L, \mathcal{E})$ :

- The centralizer of $E \in \mathcal{E}$ is an abelian vertex group of $\operatorname{JSJ}(L, \mathcal{E})$. This can be arranged by subdivision or introduction of valence one vertex groups.

- If $E, E^{\prime} \in \mathcal{E}$ have conjugate centralizers then they are contained in the same maximal abelian subgroup.

Let $\Delta$ be a GAD of a limit group $L$ and let $L_{P}$ be the subgroup of $L$ constructed by replacing each abelian vertex group by its peripheral subgroup. The envelope of a rigid vertex group $R$ of $\Delta$ is the subgroup of $L_{P}$ generated by $R$ and the centralizers of edge groups incident to $R$ in $L_{P}<L$. An automorphism of a group $G$ is internal on a subgroup $H$ if it agrees with the restriction of an inner automorphism to $H$.

The mapping class group, $\operatorname{Out}\left(\mathbb{F}_{n}\right)$, and $\operatorname{SL}(n, \mathbb{Z})$ are (virtually) generated by Dehn twists. Similarly, every GAD determines a group of automorphisms generated by visible Dehn twists.

Definition 2.5 (Modular group) Let $G=A *_{C} B$ and let $c \in Z_{B}(C)$. The Dehn twist by $c$ is the automorphism of $G$ defined by conjugation by $c$ on $B$ and the identity on $A$. Similarly, if $G$ is an HNN extension $A *_{C}$, let $t$ be the stable letter of the splitting conjugating $C$ to $C^{\prime}<A$. Let $c \in Z_{A}(C)$. The Dehn twist in $c$ is the automorphism of $G$ sending $t$ to $t c$ restricting to the identity on $A$.

${ }^{1}$ This can be slightly awkward as the normal impulse upon encountering a surjective map

$$
\text { (edge group) } \rightarrow \text { (vertex group) }
$$

is to collapse the edge. This convention makes the notation in the construction of strict homomorphisms in Section 5.2 somewhat simpler. 
Let $\Delta$ be a GAD of a group $G$. The relative modular group $\operatorname{Mod}(G, \Delta)$ is the subgroup of $\operatorname{Aut}(G)$ generated by all Dehn twists in one-edged abelian splittings of $G$ visible in $\Delta$.

The group $\operatorname{Mod}(L, \operatorname{JSJ}(L))$, or simply $\operatorname{Mod}(L)$ for convenience, is the modular group of a freely indecomposable limit group $L$.

Note that in the definition of Dehn twist the edge group $C$ need not be nontrivial. In this case there is no restriction on centralizers and we recover Nielsen moves as a special kind of Dehn twist.

\subsection{Strict}

The standard example of a nonelementary, freely indecomposable, nonabelian limit group is the double of a free group along a suitably complicated ${ }^{2}$ indivisible element. Let $F_{1}=\left\langle a_{1}, \ldots, a_{n}\right\rangle, F_{2}=\left\langle b_{1}, \ldots, b_{n}\right\rangle$, and let $\omega$ be a word in $x_{1}, \ldots, x_{n}$. Set $G=\left(F_{1} * F_{2}\right) /\left\langle\left\langle\omega\left(a_{i}\right) \omega\left(b_{i}\right)^{-1}\right\rangle\right.$. Let $\tau$ be the Dehn twist by $\omega\left(a_{i}\right)$. There is a natural retraction $r: G \rightarrow F_{1}$ and the sequence $r \circ \tau^{n}$ converges to $G$. The retraction $r$ is an example of a strict homomorphism, and in some sense every strict homomorphism is of this type: replace the one-edged splitting of $G$ by a GAD, replace $r$ by a homomorphism satisfying some simple conditions (see Theorem 2.9), and use elements of the modular group to construct convergent sequences.

Let $G$ be a group, let $\mathcal{E}$ be a collection of subgroups of $G$, and let $\operatorname{Aut}(G, \mathcal{E})$ be the subgroup of $\operatorname{Aut}(G)$ consisting of elements $\varphi$ internal on every element of $\mathcal{E}$.

Definition 2.6 (Strict [14]) Fix a finitely generated group $G$, a collection $\mathcal{E}$ of subgroups of $G$, and a limit group $L$. A homomorphism $\rho: G \rightarrow L$ is $\mathcal{E}$-strict if, given a sequence of homomorphisms $f_{n}: L \rightarrow \mathbb{F}$ converging to $L$, there exists a sequence $\varphi_{n} \in \operatorname{Aut}(G, \mathcal{E})$ such that $f_{n} \circ \sigma \circ \varphi_{n}$ converges to $G$. A $\varnothing$-strict homomorphism is simply strict. If $\sigma: G \rightarrow L$ is $\operatorname{Ell}(\Delta)-$ strict we will also say that $\sigma$ is $\Delta$-strict.

Remark 2.7 If $\Delta$ has no trivial edge groups then $\operatorname{Mod}(G, \Delta)$ is finite index in $\operatorname{Aut}(G, \operatorname{Ell}(\Delta))$, and if $\rho$ is $\operatorname{Ell}(\Delta)$-strict the sequence $\varphi_{n}$ may be chosen from $\operatorname{Mod}(G, \Delta)$. If $G$ is a freely indecomposable limit group then $\Delta$-strictness implies $\operatorname{JSJ}(G)$-strictness.

Definition 2.8 (Strict resolution [14]) A sequence of epimorphisms $L_{0} \rightarrow L_{1} \rightarrow$ $\cdots \rightarrow L_{n}$ such that each $L_{i} \rightarrow L_{i+1}$ is strict is a partial strict resolution of $L_{0}$. If $L_{n}$ is free then the sequence is a strict resolution of $L_{0}$. A (partial) strict resolution is proper if all maps are proper.

\footnotetext{
2 to avoid building a surface or a free group
} 
By [14, Proposition 5.10], limit groups have strict resolutions, but do not use this proposition in what follows. The last two bullets in the statement below are to guarantee that maps are well behaved when a GAD sees a free decomposition.

Theorem 2.9 ([14; 4]; see also [19]) Fix a finitely generated group $G$, with a GAD $\Delta$ and a map $\sigma: G \rightarrow L, L$ a limit group. The following imply that $\sigma$ is $\Delta$-strict.

- Edge groups, envelopes of rigid vertex groups, peripheral subgroups of abelian vertex groups are embedded by $\sigma$. QH vertex groups are sent to nonabelian subgroups of $L$ by $\sigma$.

- If $e$ is an edge of $\Delta$ with nontrivial edge group then at least one of the inclusions of $G_{e}$ into a vertex group of the one edged splitting of $G$ induced by $e$ is maximal abelian. This requirement is needed to guarantees that $G$ is CSA.

- If $A$ is an abelian vertex group with trivial peripheral subgroup then $\sigma$ doesn't vanish on $A$.

- The image is nonabelian.

A homomorphism $G \rightarrow H$ of freely indecomposable limit groups is strict if and only if it is $\operatorname{JSJ}(G)$ strict.

\section{Lemma 2.10 Let}

$$
L_{0} \stackrel{\sigma_{0}}{\longrightarrow} L_{1} \stackrel{\sigma_{1}}{\longrightarrow} \cdots \stackrel{\sigma_{n-1}}{\longrightarrow} L_{n}
$$

be a strict resolution of $L_{0}$. It follows from Theorem 2.9 that if $H<L_{0}$ there are automorphisms $\varphi_{i} \in \operatorname{Aut}\left(L_{i}\right)$ such that

$$
L_{0} \stackrel{\sigma_{0} \varphi_{0}}{\longrightarrow} L_{1} \stackrel{\sigma_{1} \varphi_{1}}{\longrightarrow} \cdots \stackrel{\sigma_{n-1} \varphi_{n-1}}{\longrightarrow} L_{n}
$$

restricted to $H$ is a strict resolution of $H$.

Remark 2.11 Theorem 2.9 is stated without the last two bullets in [4; 19]. They prove that a given group is a limit group and in this case trivial edge groups (which give free splittings) may be ignored: free products of limit groups are limit groups. Since we are trying to prove additionally that a given homomorphism is strict we can't argue exactly in this way. Corollary 2.13 is the special case of Theorem 2.9 where all edge groups are trivial and all vertex groups are embedded. We show only this case and leave the combination of the two proofs as an exercise for the interested reader. 
Lemma 2.12 [1] Let $g_{i}, i=0, \ldots, m$, and $t$ be nontrivial words in a free group. If $\left[g_{i}, t\right] \neq 1$ for all $i$, then for sufficiently large $\min _{i}\left\{\left|n_{i}\right|\right\}$, the word

$$
g_{0} t^{n_{1}} g_{1} \cdots g_{m-1} t^{n_{m}} g_{m}
$$

is not trivial.

Corollary 2.13 Let $\sigma: G \rightarrow H$ have nonabelian image, $H$ a limit group. Suppose $\sigma$ is injective on freely indecomposable free factors of $G$. Then $\sigma$ is strict. In particular, $G$ is a limit group.

Proof of Corollary 2.13 The strategy is to find automorphisms $\varphi_{n}$ of $G$ and a sequence of homomorphisms $f_{n}: H \rightarrow \mathbb{F}$ converging to $H$ such that $\underset{\operatorname{Ker}}{\longrightarrow}\left(f_{n} \sigma \varphi_{n}\right)=\{1\}$. Express $G$ as a most refined free product

$$
G_{1} * \cdots * G_{k-1} * G_{k} * \cdots * G_{l-1} * G_{l} * \cdots * G_{p+q}
$$

with $G_{i}$ nonabelian for $i \leq k-1$, noncyclic free abelian for $l-1 \geq i \geq k$, and $G_{i} \cong \mathbb{Z}$ for $i \geq l$. Suppose a basis element $x$ generating some $G_{i} i \geq l$ has trivial image in $H$. Let $\left\{g_{i, j}\right\}$ be a generating set for $G_{i}$. Precompose $\sigma$ by the automorphism which maps $x$ to $x g$ for some $g \in G_{1}$ and is the identity on the rest of $G$. In this way, arrange that no element of a fixed basis for the free part of a Grushko decomposition of $G$ has trivial image in $H$. Under the new map every free factor in some fixed Grushko free factorization embeds in $H$.

Let $B_{i, n}$ be the ball of radius $n$ in the Cayley graph of $G_{i}$ with respect to $\left\{g_{i, j}\right\}$. For each $n$, choose a homomorphism $f_{n}: H \rightarrow \mathbb{F}$ such that $f_{n}$ has nonabelian image and embeds $\sigma\left(B_{i, n}\right)$ for each $i$.

Suppose that $f_{n}$ has nonabelian image when restricted to $\sigma\left(G_{1}\right)$. Since $f_{n}\left(\sigma\left(G_{1}\right)\right)$ is nonabelian there is an element $c_{n}$ in the $G_{1}$ such that

$$
\left[f_{n}\left(\sigma\left(c_{n}\right)\right), f_{n}(\sigma(g))\right] \neq 1
$$

for all $g \in \bigsqcup B_{i, n}$. Fix $m$ and choose integers $m_{i}, i>1$, such that $\left|m_{i}-m_{j}\right|>m$ for $i \neq j$. Let $h=h_{1} \cdots h_{t}$ be a word in $\bigsqcup_{i} B_{i, n}$ such that for all $l, h_{l}$ and $h_{l+1}$ are contained in distinct $B_{i, n}$ 's, let $\Omega_{n, s}$ be the collection of all such words with length at most $s$, and let $i\left(h_{j}\right)$ be the index $i$ such that $h_{j} \in B_{i\left(h_{j}\right), n}$. Let $\varphi_{m}$ be the automorphism of $G$ which is the identity on $G_{1}$ and which maps $G_{i}$ to $c_{n}^{m_{i}} G_{i} c_{n}^{-m_{i}}$. The image of $h$ in $\mathbb{F}$ is

$$
f_{n} \sigma\left(c_{n}\right)^{m_{i\left(h_{1}\right)}} f_{n} \sigma\left(h_{1}\right) f_{n} \sigma\left(c_{n}\right)^{-m_{i\left(h_{1}\right)}} f_{n} \sigma\left(c_{n}\right)^{m_{i\left(h_{2}\right)}} f_{n} \sigma\left(h_{2}\right) \cdots
$$


Since $h_{i}$ and $h_{i+1}$ are contained in distinct factors of $G$, the terms

$$
f_{n} \sigma\left(c_{n}\right)^{-m_{i\left(h_{j}\right)}} f_{n} \sigma\left(c_{n}\right)^{m_{i\left(h_{j+1}\right)}}
$$

are at least $m$-th powers of the image of $c_{n}$. By Lemma 2.12, for sufficiently large $\min \left\{m_{i\left(h_{j}\right)}\right\}, f_{n} \sigma \varphi_{m}(h) \neq 1$. Since $\Omega_{n, s}$ has finitely many elements, choose $m$ large enough so that $f_{n} \sigma \varphi_{m}$ embeds $\Omega_{n, s}$. Thus we may treat $m$ as a function of $n$ and $s$. The family $\Omega_{n, s}$ exhausts $G$, and since $f_{n} \sigma \varphi_{m(n, s)}$ embeds $\Omega_{n, s}$,

$$
\underset{\operatorname{Ker}}{\longrightarrow}\left(f_{n} \sigma \varphi_{m(n, s)}\right)=\{1\}
$$

Now suppose all indecomposable factors of $G$ are free abelian. Then the image of, without loss of generality, $G_{1} * G_{2}$ is nonabelian and the restriction is an embedding.

Certain strict homomorphisms are isomorphisms. The following observation will be used repeatedly to show that maps are isomorphisms.

Lemma 2.14 Let $\varphi: G \rightarrow H$ be $\Delta$-strict. If, for all $\psi \in \operatorname{Mod}(G, \Delta)$, there exists $\psi^{\prime} \in \operatorname{Mod}(H)$ such that $\varphi \circ \psi=\psi^{\prime} \circ \varphi$ then $\varphi$ is an isomorphism.

2.2.1 Bounding strict resolutions One extremely important fact is that lengths of strict resolutions of a limit group are controlled by their ranks. This is the one place in the theory where linearity is used, and it would be very interesting to find a proof that doesn't. See Question 1 below.

Theorem 2.15 Suppose $\mathbb{F}_{N} \rightarrow L_{0} \rightarrow \cdots \rightarrow L_{k}$ is a sequence of proper strict epimorphisms of limit groups. Then $k \leq 3 N$.

If $\mathbb{F}_{N} \rightarrow L_{1} \rightarrow \cdots \rightarrow L_{k} \cong \mathbb{F}_{M}$ is a sequence of proper strict epimorphisms, then $k \leq 3(N-M)$.

The representation variety $\operatorname{Hom}(G, \operatorname{SL}(2, \mathbb{C}))$ of a finitely generated group $G$ is an affine variety and will be denoted by $\mathcal{R}(G)$. For $g \in G$, let ev $g$ denote the evaluation map $\rho \mapsto \rho(g)$.

Consider action of the modular group of a limit group $L$ on $\mathcal{R}(L)$. The image of an edge group of an abelian splitting of $L$ under a representation $\rho$ is (typically) contained in a 1 -parameter subgroup of $\operatorname{SL}(2, \mathbb{C})$, and $\rho$ may be twisted by elements of this subgroup to produce new representations. The twisted representations can be chosen to vary holomorphically in (suitable) $\rho$ and a (suitable) parameter $z$, and we conclude that the modular group fixes, componentwise, the strict subvariety of the representation variety. 
Definition 2.16 Let $G=G_{1} *_{E} G_{2}$ be a splitting over a finitely generated abelian group $E$ and let $\tau$ be the Dehn twist in $E$ by $e \in Z_{G_{2}}(E)$. Let $F_{1}=\left\langle x_{1}, \ldots, x_{n_{1}}\right\rangle$ and $F_{2}=\left\langle y_{1}, \ldots, y_{n_{2}}\right\rangle$ be free groups with homomorphisms $\lambda_{i}: F_{i} \rightarrow G_{i}$. Set $\widetilde{G}=F_{1} * F_{2}$, and let $\lambda=\lambda_{1} * \lambda_{2}: \widetilde{G} \rightarrow G$ be the obvious surjection. Choose $\tilde{e} \in \lambda_{2}^{-1}(e)$, and let $\tilde{\tau}$ of $\widetilde{G}$ to be the Dehn twist by $\tilde{e}$. Then $\tilde{\tau}$ satisfies $\lambda \tilde{\tau}=\tau \lambda$.

Let $G=G^{\prime} *_{E}$ be an HNN extension. Define $\widetilde{G}$ in the same way: Choose $F^{\prime}=$ $\left\langle x_{1}, \ldots, x_{n}\right\rangle$, a homomorphism $\lambda^{\prime}: F^{\prime} \rightarrow G^{\prime}$, and $\widetilde{G}$ the HNN extension $F^{\prime} *\langle\widetilde{t}\rangle$. Set $\lambda(\widetilde{t})=t$. Choose a lift $\tilde{e} \in \lambda^{\prime-1}(e)$ and let $\tilde{\tau}$ be the Dehn twist by $\tilde{e}$.

The exponential map exp: $M(2, \mathbb{C}) \rightarrow \mathrm{GL}(2, \mathbb{C})$ is holomorphic, converges everywhere, and is biholomorphic in a neighborhood of all points $v \neq 0 \in \mathfrak{s} l_{2} \mathbb{C} \subset M(2, \mathbb{C})$ such that $\exp (v) \neq \pm I_{2}$. See Rossman [12, Chapter 1, Example 9] for the computation in the real case.

Lemma 2.17 Define $P_{\delta}=N_{\delta}(\{x+0 i \mid 0 \leq x \leq 1\}) \subset \mathbb{C}$. For all $g \in \operatorname{SL}(2, \mathbb{C})$, $\operatorname{tr}(g) \neq-2$, there is an element $v_{g} \in \mathfrak{s} l_{2} \mathbb{C}$, a neighborhood $U_{g} \subset \operatorname{SL}(2, \mathbb{C})$, a continuous choice of logarithm $\tilde{U}_{g}=\log \left(U_{g}\right)$, a vector $v_{g} \in \tilde{U}_{g}$, and $\delta, \epsilon>0$ such that:

- $\exp \left(v_{g}\right)=g$.

- $\exp \left(P_{\delta} \cdot \tilde{U}_{g}\right) \subset \operatorname{SL}(2, \mathbb{C}) \backslash\{-I\}$.

- $\left.\exp \right|_{N_{\epsilon}\left([0,1] \cdot v_{g}\right)}$ is biholomorphic onto its image.

- $P_{\delta} \cdot \tilde{U}_{g} \subset N_{\epsilon}\left([0,1] \cdot v_{g}\right)$.

Proof The exponential map is biholomorphic on a neighborhood of each point of $[0,1] \cdot v_{g}$, and if $f: M \rightarrow M^{\prime}$ is smooth, injective on an embedded smooth, compact, submanifold $N \subset M$, and is a local diffeomorphism at every point of $N \subset M$, then $f$ is a diffeomorphism on a neighborhood of $N$.

Definition 2.18 Let $\mathcal{R}_{2}(G)$ be the union of the irreducible components $V$ of $\mathcal{R}(G)$ such that for all $g \in G$, the map

$$
\rho \mapsto(\operatorname{tr}(\operatorname{ev} g(\rho))+2)
$$

doesn't vanish on $V$.

Lemma 2.19 Let $X$ be an irreducible component of $\mathcal{R}(G)$, and let $\tau$ be a Dehn twist by $e$ as above. If, for some $\rho \in X, \operatorname{tr}(\rho(e)) \neq-2$, then $\tau(X)=X$. The modular group acts trivially on the set of irreducible components of $\mathcal{R}_{2}(G)$. 
Proof We prove the lemma for Dehn twists from amalgamations. The argument for HNN extensions is identical. Let $\widetilde{G}$ and $\widetilde{\tau}$ as in Definition 2.16, and consider the representation $\rho \circ \lambda$. Clearly $\operatorname{tr}(\rho(\lambda(\widetilde{e}))) \neq-2$.

Let $U_{\rho(\lambda(\widetilde{e}))}, \tilde{U}_{\rho(\lambda(\widetilde{e}))}$, and $P_{\delta}$ be as in Lemma 2.17, and choose a neighborhood $V$ of $\rho \circ \lambda$ so that $\operatorname{ev}_{\tilde{e}}(V) \subset U_{\rho(e)}$. Then the map $\tau_{H}: V \times P_{\delta} \rightarrow \mathcal{R}(\mathbb{F})$ defined by

$$
(\eta, z) \stackrel{\tau_{H}}{\longrightarrow}\left\{\begin{aligned}
x_{i} & \mapsto \eta\left(x_{i}\right) \\
y_{i} & \mapsto \eta\left(y_{i}\right)^{\exp (z \cdot \log (\eta(e)))}
\end{aligned}\right.
$$

is holomorphic.

Let $K_{i}=\operatorname{Ker}\left(\lambda_{i}\right)$, and let $\left\{r_{j}^{i}\right\}_{j=1, \ldots, \infty}$ be an enumeration of $K_{i}$. Since $\mathbb{C}\left[\operatorname{SL}(2, \mathbb{C})^{n}\right]$ is Noetherian, there exists $k<\infty$ such that

$$
\rho_{i} \in \mathcal{R}\left(G_{i}\right) \Longleftrightarrow \forall j \leq k\left(\mathrm{ev}_{j}^{i}\left(\rho_{i}\right)=I_{2}\right) .
$$

The inclusion $E \hookrightarrow G_{i}$ induces a restriction map $\mathcal{R}\left(G_{i}\right) \rightarrow \mathcal{R}(E)$. Now $G$ is the pushout of the diagram $\left\{E \hookrightarrow G_{i}\right\}$, therefore $\mathcal{R}(G)$ is the pullback of the diagram $\left\{\mathcal{R}\left(G_{i}\right) \rightarrow \mathcal{R}(E)\right\}$, and we identify $\mathcal{R}(G)$ with the set of pairs $\left(\rho_{1}, \rho_{2}\right) \in$ $\mathcal{R}\left(G_{1}\right) \times \mathcal{R}\left(G_{2}\right)$ such that the restrictions $\left.\rho_{1}\right|_{E}$ and $\left.\rho_{2}\right|_{E}$ agree. Since $E$ is finitely generated there are elements $g_{i}^{l} \in F_{i}, l=1, \ldots, m$, corresponding to generators of $E$, such that

$$
\rho=\left(\rho_{1}, \rho_{2}\right) \in \mathcal{R}(G) \quad \Longleftrightarrow \quad \forall l\left(\operatorname{evg}_{l}^{1}\left(\rho_{1}\right)=\operatorname{ev}_{g_{l}^{2}}\left(\rho_{2}\right)\right)
$$

for $\rho_{i} \in \mathcal{R}\left(G_{i}\right)$. The family $\tau_{H}$ clearly preserves the relations $\mathrm{ev}_{r_{i}^{j}}$ and since $e \in$ $Z_{G_{2}}(E)$, we have for all $g_{l}^{2}, \rho_{2} \in \mathcal{R}\left(G_{2}\right)$,

$$
\rho_{2}\left(g_{l}^{2}\right)^{\exp (z \cdot \log (\eta(e)))}=\rho_{2}\left(g_{l}^{2}\right) .
$$

Therefore $\tau_{H}(V \cap \mathcal{R}(G)) \subset \mathcal{R}(G)$. Choose $V$ so that $W=V \cap X$ is irreducible. Then $W \times P_{\delta}$ is also irreducible, and since $\tau_{H}$ doesn't change the values of this finite set of equations, $\tau_{H}\left(W \times P_{\delta}\right) \subset \mathcal{R}(G)$. The image of an irreducible complex analytic variety under a holomorphic map has irreducible closure, and since $W \nsubseteq \overline{\mathcal{R}(G) \backslash X}$, $\tau_{H}\left(W \times P_{\delta}\right) \subset X$. Since $\tau_{H}(W \times\{1\})=\tau(W)$ we have $\tau(X)=X$.

Definition 2.20 Let $\mathcal{R}_{S}(L)$ be the union of irreducible components $X$ of $\mathcal{R}(L)$ such that there is a $\rho \in \mathcal{R}(\mathbb{F})$, with $\operatorname{tr}(\rho(g)) \neq-2$ for all $g$, and a sequence $f_{n}: L \rightarrow \mathbb{F}$ converging to $L$ such that $\rho \circ f_{n} \in X$.

The variety $\mathcal{R}_{s}(L)$ is nonempty, and, by definition, $\mathcal{R}_{s}(L) \subset \mathcal{R}_{2}(L)$.

Lemma 2.21 If $\sigma: L \rightarrow L^{\prime}$ is strict then $\mathcal{R}_{s}\left(L^{\prime}\right) \subset \mathcal{R}_{S}(L)$ and if $X^{\prime} \subset X$ are irreducible components of $\mathcal{R}_{s}\left(L^{\prime}\right)$ and $\mathcal{R}(L)$, respectively, then $X \subset \mathcal{R}_{S}(L), \operatorname{dim} X>$ $\operatorname{dim} X^{\prime}$, and $\operatorname{dim} \mathcal{R}_{S}(L)>\operatorname{dim} \mathcal{R}_{S}\left(L^{\prime}\right)$. 
Proof To prove the first part of the claim, choose an irreducible component $X^{\prime}$ of $\mathcal{R}_{S}\left(L^{\prime}\right)$ and an irreducible component $X$ of $\mathcal{R}(L)$ containing $X^{\prime}$. We show that $X \subset \mathcal{R}_{s}(L)$. Clearly $X^{\prime} \subset \mathcal{R}_{2}(L)$.

Choose a sequence $f_{n} \in \operatorname{Hom}\left(L^{\prime}, \mathbb{F}\right)$ converging to $L^{\prime}$, and $\rho \in \mathcal{R}(\mathbb{F})$ with no traces of images of elements -2 , such that $\rho \circ f_{n}$ is contained $X^{\prime}$ for all $n$. Choose $\varphi_{n} \in \operatorname{Mod}(L)$ such that $g_{n}=f_{n} \circ \sigma \circ \varphi_{n}$ converges to $L$. By Lemma $2.19 \varphi_{n}$ fixes $X$ setwise and we have $\rho \circ g_{n} \in X$ for all $n$, hence $X \subset \mathcal{R}_{S}(L)$.

If $1 \neq g \in \operatorname{Ker}(\sigma)$ then $\operatorname{ev}_{g}$ doesn't vanish on $X$ but it does vanish on $X^{\prime}$, hence $\operatorname{dim} X^{\prime}<\operatorname{dim} X$.

Lemma 2.21 and that $\operatorname{dim} \mathcal{R}\left(\mathbb{F}_{n}\right)=3 n$ imply Theorem 2.15 .

\subsection{Analysis lattices}

Definition 2.22 [14, Section 4] The principal cyclic analysis lattice of a limit group $L$ is the tree of groups constructed as follows:

- The root node is $L$ itself.

- Let $G=G_{1} * \ldots * G_{p} * \mathbb{F}_{q}$ be a Grushko decomposition of a group in the principal cyclic analysis lattice. If $G$ is not elementary, the children of $G$ are $\mathbb{F}_{q}$, the elementary freely indecomposable free factors of $G$, and the vertex groups of the principal cyclic JSJ decompositions of the nonelementary freely indecomposable free factors.

- Elementary limit groups are terminal nodes of the tree.

Definition 2.23 The depth of a limit group $H$, depth $(H)$, is the number of levels in its principal cyclic analysis lattice.

The width of $H$ is the maximum number of groups appearing at any level of the principal cyclic analysis lattice.

The cyclic JSJ of $L$ is the JSJ decomposition associated to the family of all one-edged root-closed cyclic splittings in which all noncyclic abelian subgroups are elliptic, and the cyclic analysis of a limit group is constructed like the principal cyclic analysis lattice, but instead by passing to vertex groups in the cyclic JSJ at each level. We point out, but do not use, the following theorem of Sela's.

Theorem 2.24 [14, Proposition 4.3] The depth of the cyclic analysis lattice of a limit group is controlled by its first betti number. 
The cyclic analysis lattice may be shorter than the principal cyclic analysis lattice.

Theorem 2.25 The depth of a limit group $L$ is bounded by $3 \operatorname{rk}(L)$.

Proof Let $L=L_{1} \rightarrow L_{2} \rightarrow \cdots \rightarrow L_{n \leq 3 \mathrm{rk}(L)}$ be a strict resolution of $L$, and let $H$ be a freely indecomposable free factor of $L$, and let $H=K_{1} \rightarrow K_{2} \rightarrow \cdots H$ be the strict resolution of $H$ obtained by restricting to the images of $H$ (Lemma 2.10). Let $V$ be a vertex group of $\operatorname{PJSJ}(H)$, and consider a freely indecomposable free factor $W$ of $V$. Since the edge groups of $\operatorname{PJSJ}(L)$ contained in the induced decomposition of $W$ are elliptic in $\mathrm{JSJ}\left(L_{2}\right)$, the envelopes of rigid vertex groups of $\mathrm{JSJ}(W)$ are contained in envelopes of rigid vertex groups of $L_{2}$, and are embedded in $K_{3}$ under the map $K_{2} \rightarrow K_{3}$. Thus the sequence $W \rightarrow K_{3} \rightarrow \cdots$, restricted to the images of $W$ is a strict resolution. By induction on the length of a shortest strict resolution, $W$ has depth at most $3 \operatorname{rk}(L)-1$. Since $W$ is at the second level of the analysis lattice of $L, L$ has depth at most $3 \mathrm{rk}(L)$.

Lemma 2.26 Let $L$ be a nonabelian limit group and let $E<L$ be an abelian subgroup. Then $\mathrm{b}_{1}(L, E) \geq 1$.

Proof The lemma clearly holds for nonabelian free groups, and the image of $E$ under a map $L \rightarrow \mathbb{F}$ is cyclic.

Lemma 2.27 The numbers of edges and vertex groups in the principal cyclic and abelian JSJ decompositions of a freely indecomposable limit group are controlled by its first betti number.

Denote by Verts $(b)$ the maximal number of vertex groups in the abelian JSJ of a freely indecomposable nonelementary limit group with first betti number at most $b$.

Proof The statement for the principal cyclic JSJ follows immediately from the case of the abelian JSJ. Let $L$ be a freely indecomposable limit group.

By Lemma 2.26 valence one vertex groups have nonzero first betti number, relative to their incident edge groups, and the underlying graph of the JSJ then has at most $\mathrm{b}_{1}(L)$ valence one vertex groups, and the first betti number of the underlying graph is bounded by $\mathrm{b}_{1}(L)$, it suffices to bound the lengths of segments of adjacent valence two vertex groups. Let $R$ be a rigid vertex group. At least one edge group incident to $R$ is infinite cyclic, otherwise $R$ has an abelian splitting relative to incident edge groups which can be used to refine the JSJ decomposition. Let $v_{i}$ be a sequence of adjacent valence two vertices, and let $e_{i}$ be the edge connecting $v_{i}$ to $v_{i+1}$. If $e_{j}$ is not 
infinite cyclic then at most one of $e_{j-1}$ or $e_{j+1}$ is not infinite cyclic, and by collapsing noncyclic edges the number of vertex groups is reduced by at most a factor of $\frac{1}{3}$. A row of $n$ adjacent nonabelian limit groups connected by infinite cyclic edges has first betti number at least $n-3$ relative to incident edges, and the number of disjoint rows of length at least four in the collapsed decomposition is at most $\mathrm{b}_{1}(L)$.

To apply the uniform bound on the length of a strict resolution provided by Theorem 2.15, especially in Section 4, the rank of a limit group has to be controlled by its first betti number and depth. A straightforward induction using Lemma 2.27 and presentations compatible with graphs of groups decompositions gives the following.

Lemma 2.28 The rank of a limit group is controlled by its depth and first betti number.

\section{Chains, resolutions, complexities}

A sequence of limit groups is a finite sequence

$$
\mathcal{L}=\left(\ldots, \mathcal{L}\left(j_{i}\right), \mathcal{L}\left(j_{i+1}\right), \ldots\right),
$$

with $j_{i}<j_{i+1}$, of limit groups, equipped with homomorphisms

$$
\varphi_{m, n}: \mathcal{L}(m) \rightarrow \mathcal{L}(n)
$$

such that

$$
\varphi_{n, l} \circ \varphi_{m, n}=\varphi_{m, l}
$$

for all $m<n<l$. Sometimes we ignore the fact that a sequence isn't necessarily indexed by adjacent natural numbers, and simply refer to the $i$-th element of a sequence $\mathcal{L}$ as $\mathcal{L}(i)$. A sequence is a chain if all the maps are epimorphisms. A limit group $L$ determines a variety $\operatorname{Hom}(L, \mathbb{F})$. We use this terminology because the dual sequence of inclusions of Hom-sets

$$
\cdots \supset \operatorname{Hom}(\mathcal{L}(i), \mathbb{F}) \supset \operatorname{Hom}(\mathcal{L}(i+1), \mathbb{F}) \supset \cdots
$$

is a chain of varieties, therefore sequences of epimorphisms of limit groups originating from $L$ correspond to chains of irreducible subsets of $\operatorname{Hom}(L, \mathbb{F})$. All chains of irreducible varieties are of this form, which explains the term Krull dimension.

The length of a chain $\mathcal{L}$ is denoted $\|\mathcal{L}\|$. The proper length of a chain $\mathcal{L},\|\mathcal{L}\|_{\mathrm{pl}}$, is the number of indices $n$ such that $\mathcal{L}(n-1) \rightarrow \mathcal{L}(n)$ is not an isomorphism. The rank of a group $G, \operatorname{rk}(G)$, is the minimal number of elements needed to generate $G$. The rank of a chain is the rank of the initial group: $\operatorname{rk}(\mathcal{L}):=\operatorname{rk}(\mathcal{L}(1))$. The first betti number of a chain is defined in the same way and is denoted by $b_{1}(\mathcal{L})$. 
Let $\mathcal{L}$ and $\mathcal{G}$ be sequences of limit groups. A morphism $\rho: \mathcal{G} \rightarrow \mathcal{L}$ is a sequence of homomorphisms $\rho_{j}: \mathcal{G}(j) \rightarrow \mathcal{L}\left(i_{j}\right)$, such that if $j<j^{\prime}$ then $i_{j}<i_{j^{\prime}}$, with $\varphi_{i_{j}, i_{k}} \circ \rho_{j}=$ $\rho_{k} \circ \varphi_{j, k}$ for all $j$ and $k$. Composition of morphisms of sequences is defined in the obvious way. A morphism $\rho$ is injective (surjective) if $\rho_{j}$ is injective (surjective) for all $j$. Injections and surjections are written using the using the usual typographical conventions. The image of $\rho: \mathcal{G} \rightarrow \mathcal{L}$ is the sequence $\operatorname{Im} \mathcal{G}(j):=\rho_{j}(\mathcal{G}(j))$, with maps obtained by restricting maps from $\mathcal{L}$. Any $\rho: \mathcal{G} \rightarrow \mathcal{L}$ factors as $\mathcal{G} \rightarrow \operatorname{Im} \mathcal{G} \hookrightarrow \mathcal{L}$. If $\mathcal{L}^{\prime} \rightarrow \mathcal{L}$ is both injective and surjective then $\mathcal{L}^{\prime}$ is a subsequence of $\mathcal{L}$. We write this as $\mathcal{L}^{\prime}<\mathcal{L}$.

Definition 3.1 (Depth of a map of chains) For each $j$ let $\mathcal{L}_{\rho}\left(i_{j}\right)$ be the lowest node in the principal cyclic analysis lattice of $\mathcal{L}\left(i_{j}\right)$ containing a conjugate of $\rho_{j}(\mathcal{G}(j))$. The depth of $\rho$ is $\operatorname{depth}(\rho):=\max _{j}\left\{\operatorname{depth}\left(\mathcal{L}_{\rho}\left(i_{j}\right)\right)\right\}$.

Definition 3.2 (Resolution) A resolution of a chain $\mathcal{L}$ is a chain $\mathcal{H}$, indexed by $j$, a pair of tuples of maps $\rho_{j}: \mathcal{H}(j) \rightarrow \mathcal{L}\left(i_{j}\right)$ and $\psi_{j}: \rho_{j}\left(\mathcal{L}\left(i_{j}\right)\right) \rightarrow \mathcal{H}(j+1)$, when indices are defined, such that the diagrams

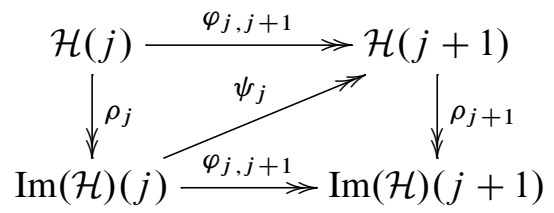

commute.

If $\mathcal{H}$ is a resolution of $\mathcal{L}$, then we write $\mathcal{H} \square_{\rho} \mathcal{L}$, possibly without the subscript. The symbol " $\square$ " is supposed to evoke the commutative diagram above. The depth of the resolution $\mathcal{H} \nabla_{\rho} \mathcal{L}$ is the depth of $\rho$. A resolution $\mathcal{H} \nabla_{\rho} \mathcal{L}$ is surjective if all $\rho_{j}$ and $\psi_{j}$ are surjective. Maps will typically be unnamed unless there is some risk of confusion.

Suppose $\mathcal{H} \square \mathcal{L}$ and $R<\mathcal{H}(n)$. The sequence of images $\mathcal{R}^{n}$ defined by

$$
\mathcal{R}^{n}(m):=\varphi_{n, m}(R)<\mathcal{H}(m), \quad m \geq n,
$$

is a resolution with maps induced by restriction.

The complexity of $\mathcal{H} \square_{\rho} \mathcal{L}, \operatorname{Comp}\left(\mathcal{H} \square_{\rho} \mathcal{L}\right)$, is the pair $\left(\mathrm{b}_{1}(\mathcal{H}), d(\rho)\right)$, with the partial order given by comparison in both coordinates. The length and proper length of $\mathcal{H} \square \mathcal{L}$ are the length and proper length of $\mathcal{H}$, respectively.

Sequences of minimal complexity $(b, 1)$ are the base case of the induction. Groups in minimal complexity sequences are elementary limit groups. Theorem 1.1 obviously holds for sequences of minimal complexity: limit groups are Hopf, and there are only finitely many with a given first betti number. 
Lemma 3.3 (Minimal depth sequences) Sequences of proper epimorphisms of free products of elementary limit groups have length controlled by the first betti number.

This proposition is not really necessary since the analysis of sequences of minimal complexity is a subcase of our more general analysis of sequences of resolutions of limit groups and the observation that any map with nonabelian image from a nonabelian elementary limit group to another limit group is strict.

Theorem 1.1 can be reformulated in terms of resolutions.

Theorem 3.4 $\|\mathcal{H}\|_{\mathrm{pl}}$ is controlled by $\operatorname{Comp}\left(\mathcal{H} \square_{\rho} \mathcal{L}\right)$.

Theorem 1.1 follows immediately from Theorem 3.4 since

$$
\operatorname{Comp}\left(\mathcal{L} \square_{\mathrm{id}} \mathcal{L}\right) \leq(\operatorname{rk}(\mathcal{L}), 3 \operatorname{rk}(\mathcal{L})) .
$$

The converse follows from Lemma 2.28.

\section{Degenerate maps}

In this section we construct a resolution of $\mathcal{L}$ such that every composition of maps from $\mathcal{H} \square \mathcal{L}$ with codomain in $\mathcal{H}$ is as far from strict as possible.

Definition 4.1 (Degenerate) An epimorphism $\varphi: L \rightarrow L^{\prime}$ of limit groups has a strict factorization if there is a quotient limit group $\psi: L \rightarrow L_{s}$ and a strict morphism $\varphi_{s}: L_{s} \rightarrow L^{\prime}$ such that $\varphi_{s} \circ \psi=\varphi$. An epimorphism $\varphi: L \rightarrow L^{\prime}$ is degenerate if it has no proper strict factorizations. A pair of epimorphisms

$$
A \stackrel{\pi}{\rightarrow} B \stackrel{\pi^{\prime}}{\rightarrow} C
$$

has a strict factorization if, for all factorizations

$$
B \rightarrow L_{m} \rightarrow \cdots \rightarrow L_{1} \rightarrow C
$$

of $\pi^{\prime}$, such that

$$
L_{m} \rightarrow \cdots \rightarrow L_{1} \rightarrow C
$$

is a partial strict resolution of $L_{m}$, the induced map $A \rightarrow L_{m}$ has a proper strict factorization.

A pair

$$
A \stackrel{\pi}{\rightarrow} B \stackrel{\pi^{\prime}}{\rightarrow} C
$$


is degenerate if it has no proper strict factorizations. In particular there is a factorization

$$
B \rightarrow L_{m} \rightarrow \cdots \rightarrow L_{1} \rightarrow C
$$

of $\pi^{\prime}$ such that $A \rightarrow L_{m}$ is degenerate. Note that this implies that $B \rightarrow L_{m}$ is also degenerate.

A chain is degenerate if all maps from the chain are degenerate. A resolution $\mathcal{H} \square \mathcal{L}$ is maximal if, for all $j<j^{\prime}$, the maps $\mathcal{H}(j) \rightarrow \mathcal{H}\left(j^{\prime}\right)$ and $\operatorname{Im}(\mathcal{H})(j) \rightarrow \mathcal{H}\left(j^{\prime}\right)$ are degenerate.

Example 4.2 Let $F$ be a finitely generated free group, $g \in F$ indivisible, and let $\varphi: F \rightarrow G$ be a noninjective map of limit groups such that $\varphi(g)$ is indivisible and has cyclic centralizer, and such that $\operatorname{JSJ}\left(F^{\prime},\{\langle\pi(g)\rangle\}\right)$ is trivial for all factorizations

$$
F \stackrel{\pi}{\rightarrow} F^{\prime} \stackrel{\varphi^{\prime}}{\rightarrow} G
$$

of $\varphi$ (including $F$ and $G$ ). Let $H$ be the double of $F$ along $g$ and let $H^{\prime}$ be the double of $G$ along $\varphi(g)$. The natural map $H \rightarrow H^{\prime}$ is probably degenerate.

Other simple examples include the map of a nonabelian limit group $L$ onto $\mathrm{H}_{1}(L)$ (modulo torsion).

Theorem 4.3 There is a function $M=M(K, n)$, such that if $\mathcal{L}$ is a chain with $\|\mathcal{L}\|_{\mathrm{pl}}>M(K, \operatorname{rk}(\mathcal{L})), K>3 \operatorname{rk}(\mathcal{L})$, then there is a surjective maximal resolution $\mathcal{H} \square \mathcal{L}$ with $\|\mathcal{H}\|_{\mathrm{pl}}=K$.

Proof Let $I$ be the index set of $\mathcal{L}$. Color a triple of indices $i<j<k$ green if $\mathcal{L}(i) \rightarrow \mathcal{L}(j) \rightarrow \mathcal{L}(k)$ is degenerate and red otherwise. By Theorem 2.15 and the definition, monochromatic subsets of $I$ of size at least $3 \operatorname{rk}(\mathcal{L})$ are green. By the Ramsey theorem there is a function $R(K)$ such that if $|I| \geq R(K)$ there is a subset $I^{\prime}=\left\{i_{j}\right\}_{j=1, \ldots, K}$ of $I$ of size $K$ such that every triple in $I^{\prime}$ is monochromatic. For each $i_{j} \in I^{\prime}, j>1$, let

$$
\mathcal{L}\left(i_{j-1}\right) \rightarrow L_{m_{j}} \rightarrow \cdots \rightarrow L_{1} \rightarrow \mathcal{L}\left(i_{j}\right)
$$

be a strict resolution of $L_{m_{j}}$ provided by the definition. Set $\mathcal{H}(j)=L_{m_{j}}$, and make $\mathcal{H}$ into a chain by composing maps; $\mathcal{H} \square \mathcal{L}$ and $\mathcal{H}$ are clearly degenerate.

Let $G$ be an elementary limit group, and suppose that $\varphi: G \rightarrow H$ is degenerate. If $H$ is nonabelian then $\varphi$ is an isomorphism, and if $H$ is abelian there is nothing more to say. 


\section{Constructing strict homomorphisms}

So far we have only used strictness/degeneracy in a purely formal way. In this section we use Theorem 2.9 as a template for factoring a homomorphism $G \rightarrow H$ of limit groups through a strict map $\boldsymbol{S}(G) \rightarrow H$. In Section 6 we construct a complexity "sc" which is nondecreasing under degenerate maps, takes a number of values controlled by the first betti number, and, most importantly, if equality holds then the map essentially respects JSJ decompositions. Combined with Theorem 4.3, this gives a method for aligning JSJ decompositions in resolutions: given a chain there is a degenerate resolution which is a "graph of sequences" of limit groups over sequences of abelian edge groups.

Example 5.1 Let $\varphi: G \rightarrow H$ be a homomorphism from a finitely generated group with a one edged abelian splitting $G=G_{1} *_{E} G_{2}$ to a limit group. Let $e \in E$ and let $\tau$ be the generalized Dehn twist in by $e$. Consider a sequence of homomorphisms $f_{n}: H \rightarrow \mathbb{F}$ converging to $H$. Let $g_{n}$ be the sequence $f_{n} \circ \varphi \circ \tau^{m(n)}$. We choose $m(n)$ later.

Pass to a convergent subsequence of $g_{n}$, let $S(G)$ be the quotient of $G$ by the stable kernel, and let $\eta: G \rightarrow S(G)$ be the quotient map. If $E$ has trivial image in $H$ then we define $\boldsymbol{S}(G):=\varphi\left(G_{1} / E\right) * \varphi\left(G_{2} / E\right)$. The Dehn twist $\tau$ still pushes forward to an automorphism $\tau^{\prime}$ of $\boldsymbol{S}(G)$. If one of $G_{1}$ or $G_{2}$ has abelian image in $H$ then $\tau$ also pushes forward to $\boldsymbol{S}(G)$.

Suppose that $\tau$ pushes forward to an automorphism $\tau^{\prime}$ of $\boldsymbol{S}(G)$ and that neither $G_{1}$ nor $G_{2}$ has abelian image in $H$. Then $\varphi \circ f_{n}=g_{n} \circ\left(\tau^{\prime}\right)^{-m(n)} \circ \eta, \operatorname{ker}(\eta)<\operatorname{ker}(\varphi)$, and there is an induced strict homomorphism $\boldsymbol{S}(\varphi): \boldsymbol{S}(G) \rightarrow H$ whose composition with $\eta$ is $\varphi$. If $m(n)$ is sufficiently large, the limiting action of $S(G)$ on the $\mathbb{R}$-tree for the sequence $g_{n}$ is simplicial, induces a graph of groups decomposition $\Delta$ of $\boldsymbol{S}(G)$ with one edge, and $G_{1}$ and $G_{2}$ both have elliptic images in $\Delta$ : the graph of groups decomposition has the form $\bar{G}_{1} * E^{\prime} \bar{G}_{2}, G_{1}$ maps to the envelope of $\bar{G}_{1}, G_{2}$ maps to the envelope of $\bar{G}_{2}, E$ maps to the centralizer of $E^{\prime}$ and $\eta$ respects incidence and conjugacy data of graphs of groups, hence, $\tau$ pushes forward to $\tau^{\prime}$.

Example 5.2 As a warm-up to Theorem 5.23 we give a simple example illustrating the method used there to construct strict homomorphisms. Let $G$ be a group, $\Delta$ a representation of $G$ as an amalgam of the form $G \cong R *\langle e\rangle S$ with nonabelian vertex groups over a cyclic subgroup. Suppose there is a limit group $H$ and a map $\varphi: G \rightarrow H$ that embeds $R$ and $S$. Then $R$ and $S$ are limit groups. Suppose further that $\varphi$ embeds $R *\langle e\rangle Z_{S}(\langle e\rangle)$ and $Z_{R}(\langle e\rangle) *\langle e\rangle S$. Then $\varphi$ is strict, and $G$ is a limit group. There is a process which outputs a limit group $S(G)$, taking $\Delta$-almost-strict $\varphi: G \rightarrow H$ 
and producing a factorization $G \rightarrow S(G) \rightarrow H$, such that the composition is $\varphi$, $\boldsymbol{S}(G)$ splits over the centralizer of the image of $\langle e\rangle$, and $\boldsymbol{S}(G) \rightarrow L$ is strict. See [4, Lemma 7.9]. Let $G=G_{0}$. Define for

- $\quad$ odd $i: G_{i}=R_{i-1} * Z_{R_{i-1}}(\langle e\rangle) S_{i}$, where

$$
S_{i}:=\operatorname{Im}_{H}\left(Z_{R_{i-1}}(\langle e\rangle) * Z_{S_{i-1}(\langle e\rangle)} S_{i-1}\right) .
$$

- even $i: G_{i}=R_{i} * Z_{S_{i-1}}(\langle e\rangle) S_{i-1}$, where

$$
R_{i}:=\operatorname{Im}_{H}\left(R_{i-1} * Z_{S_{i-1}}(\langle e\rangle) Z_{S_{i}}(\langle e\rangle)\right) .
$$

This process terminates in finite time and the direct limit group is a limit group: The sequence of quotients $G_{0} \rightarrow G_{1} \rightarrow \cdots$ embeds edge groups at every step. Abelian subgroups of limit groups are finitely generated and free, and therefore satisfy the ascending chain condition, which implies that the process stabilizes in finite time. We call the direct limit $\boldsymbol{S}(G)$. The natural map $\boldsymbol{S}(G) \rightarrow H$ is strict.

These examples are intended to motivate our alignment of JSJ decompositions approach to Theorem 1.1: if $G \rightarrow H$ is degenerate we learn something about the JSJ of $H$ from the JSJ of $G$. One of three things can happen to a one-edged splitting of $G$ : the homomorphism factors through a free product given by killing the edge group and $H$ is either a free product or has lower rank, a vertex group has abelian image and $H$ splits as $H^{\prime} *_{E} A, A$ abelian, or $H$ splits, with nonabelian vertex groups, over the centralizer of the image of the edge group. The first example uses limiting actions to show how the codomain of a degenerate map inherits splittings from the domain. The information forces us to manually construct the group $S(G)$ (hence $H$, in the case of a degenerate map) from the data $G, \varphi$, and $\Delta$, as in the second example.

\subsection{Freely decomposable groups}

In this subsection, given a map $\varphi: G \rightarrow H$, we construct a strict factorization $\boldsymbol{S}(\varphi): \boldsymbol{S}(G) \rightarrow H$ and a complexity $c_{\mathrm{fd}}$ which is nonincreasing under $G \rightarrow \boldsymbol{S}(G)$. In particular, if $\varphi$ is degenerate then $c_{\mathrm{fd}}(G) \geq c_{\mathrm{fd}}(H)$. If equality holds then $G$ and $H$ have the "same" Grushko decompositions (Theorem 5.4).

Definition 5.3 [13] Let $G_{1} * \cdots * G_{p} * \mathbb{F}_{q}$ be a Grushko decomposition of a finitely generated group $G$. The Scott complexity of $G$ is the lexicographically ordered pair $\operatorname{sc}(G):=(p+q, q)$.

Let $\varphi: G \rightarrow H$. Then

$$
\operatorname{sc}(\varphi):=\max \{\operatorname{sc}(G / K) \mid K<\operatorname{Ker}(\varphi)\} .
$$


A map $G \rightarrow H$ is indecomposable if it doesn't factor through any free product, that is, it has complexity $(1,0)$. The domain and codomain of an indecomposable epimorphism are freely indecomposable.

Fix $\varphi: G \rightarrow H$ and let $G_{1} * \cdots * G_{p_{G}} * \mathbb{F}_{q_{G}}$ be a Grushko decomposition of $G$. For each freely indecomposable free factor $G_{i}$ of $G$, let $L^{i}$ be a group with highest Scott complexity that $\left.\varphi\right|_{G_{i}}: G_{i} \rightarrow \varphi\left(G_{i}\right)$ factors through. Set

$$
\boldsymbol{A S}(G):=L^{1} * \cdots * L^{p_{G}} * \mathbb{F}_{q_{G}}
$$

Each $L^{i}$ has a Grushko decomposition $L_{1}^{i} * \cdots * L_{p_{i}}^{i} * \mathbb{F}_{q_{i}}$; replace each $L_{j}^{i}$ by its image (which is necessarily freely indecomposable) in $H$ and call the resulting group $\boldsymbol{S}(G)$. There is an induced map $\boldsymbol{S}(\varphi): S(G) \rightarrow H$.

Theorem 5.4 (See Swarup [18].) Let $G \rightarrow H$ be a degenerate map of nonabelian limit groups. Then $\operatorname{rk}(G) \geq \operatorname{rk}(H)$. If $\operatorname{rk}(G)=\operatorname{rk}(H)$ then $\operatorname{sc}(G) \leq \operatorname{sc}(H)$. Keeping the notation from above, if $\operatorname{sc}(G)=\operatorname{sc}(H)$ then $p_{i}=1, q_{i}=0$, and none of the maps $G_{i} \rightarrow \operatorname{Im}\left(G_{i}\right)$ has trivial image or factors through a free product.

Proof Certainly $\operatorname{rk}(G) \geq \operatorname{rk}(H)$. If some $p_{i}=0$ (in the notation above) then $\operatorname{rk}(G)>$ $\operatorname{rk}(H)$. Similarly, if some $L_{j}^{i}$ has trivial image in $H$ then $\operatorname{rk}(G)>\operatorname{rk}(H)$, thus all $L_{j}^{i}$ have nontrivial image, and since $\operatorname{sc}\left(L^{i}\right)$ achieves $\operatorname{sc}\left(\left.\varphi\right|_{G_{i}}\right)$ each $L_{j}^{i}$ has freely indecomposable image in $H$.

Since $\boldsymbol{S}(G) \rightarrow H$ is injective on freely indecomposable free factors and has nonabelian image, by Corollary 2.13 it is strict, and since $\varphi$ is degenerate, $\boldsymbol{S}(\varphi)$ is an isomorphism. Thus $p_{H}=\sum_{i} p_{i}$ and $q_{H}=q_{G}+\sum_{i} q_{i}$. Computing that the Scott complexity of $H$ is at least that of $G$ is reduced to the straightforward computation:

$$
p_{H}+q_{H}=\sum_{i} p_{i}+q_{G}+\sum_{i} q_{i} .
$$

Since each $p_{i} \geq 1, p_{H} \geq p_{G}$ and $q_{H} \geq q_{G}$. Thus $p_{H}+q_{H} \geq p_{G}+q_{G}$. If there is equality in the first coordinate then $p_{i}$ must be 1 for all $i$, and if this and equality in the second coordinate, then $q_{i}=0$ for all $i$. Thus $\operatorname{sc}(G) \leq \operatorname{sc}(H)$, with equality only if $\operatorname{sc}\left(\left.\varphi\right|_{G_{i}}\right)=(1,0)$.

Set $c_{\mathrm{fd}}(G):=\left(\operatorname{rk}(G), \mathrm{b}_{1}(G),-\operatorname{sc}(G)\right)$. By Theorem 5.4, if $G \rightarrow H$ is degenerate and $c_{\mathrm{fd}}(G)=c_{\mathrm{fd}}(H)$ then $H \cong *_{i} \operatorname{Im}\left(G_{i}\right) * \mathbb{F}_{q}$ and the maps $G_{i} \rightarrow \operatorname{Im}\left(G_{i}\right)$ are indecomposable and degenerate.

A chain $\mathcal{L}$ of limit groups is indecomposable if each $\mathcal{L}(i) \rightarrow \mathcal{L}(j)$ is indecomposable. By Theorem 4.3 there are degenerate resolutions. Since the number of values $c_{\mathrm{fd}}$ 
can take is controlled by the rank, we have the following alignment theorem for free factorizations.

Theorem 5.5 (Reduction to indecomposable sequences) There is a function $M=$ $M(K, N)$, such that if $\mathcal{L}$ is a chain, $\|\mathcal{L}\|_{\mathrm{pl}} \geq M(K, \operatorname{rk}(\mathcal{L}))$, then there is a maximal surjective resolution $\tilde{\mathcal{L}} \square \mathcal{L}$ such that $\|\widetilde{\mathcal{L}}\|_{\mathrm{pl}}>K$ and $c_{\mathrm{fd}}$ is constant along $\tilde{\mathcal{L}}$.

In particular, $\tilde{\mathcal{L}}$ splits as a graded free product of sequences

$$
\widetilde{\mathcal{L}}=\widetilde{\mathcal{L}}_{1} * \cdots * \widetilde{\mathcal{L}}_{p} * \mathcal{F},
$$

where $\mathcal{F}$ is the constant sequence $\left(\mathbb{F}_{q}\right)$ for some $q$. The sequence $\tilde{\mathcal{L}}_{i} \square \mathcal{L}$ is an indecomposable maximal resolution of its image.

\subsubsection{Note on existence of indecomposable covers}

Definition 5.6 Let $G$ be a finitely generated freely indecomposable group, and suppose that $\varphi: H \rightarrow G$ doesn't factor through a nontrivial free product. If $H$ is finitely presented then $H$ is an indecomposable cover of $G$.

The existence of indecomposable covers of one-ended finitely generated groups follows from this kind of argument. Let $G$ be finitely generated, one-ended, and let $\mathcal{G}$ be a sequence of finitely presented groups of minimal rank such that $\lim _{\longrightarrow} \mathcal{G}(i)=G$. Call a map $A \rightarrow B$ strict if it embeds freely indecomposable free factors, and define strict factorizations, degenerate maps, etc., exactly as in Definition 4.1. Then there is a "resolution" $\mathcal{H} \square \mathcal{G}$ such that all maps with codomain in $\mathcal{H}$ are degenerate. (This time we use the infinite version of the Ramsey theorem, but still arguing exactly as in Theorem 4.3.) Arguing as in Theorem 5.4, $\operatorname{sc}(\mathcal{H}(i)) \leq \operatorname{sc}(\mathcal{H}(i+1))$, with equality only if $\mathcal{H}(i) \rightarrow \mathcal{H}(i+1)$ respects Grushko decompositions. Since $\lim \mathcal{G}(i)=G$ and $\mathcal{H}$ is a resolution of $\mathcal{G}$ we have $\lim \mathcal{H}(i)=G$ as well $(\mathcal{G}$ and $\mathcal{H}$ are cofinal). Since $G$ is freely indecomposable eventually $\operatorname{sc}(\mathcal{H}(i))=(1,0)$. Since the map $\mathcal{H}(i) \rightarrow \mathcal{G}\left(j_{i}\right)$ factors through a strict resolution, eventually $\mathcal{H}(i) \rightarrow \mathcal{G}\left(j_{i}\right)$ is an isomorphism. If $\mathcal{H}(i) \rightarrow G$ factors through a free product for some large $i$ then it factors through a finitely presented free product, but then $\mathcal{H}(i) \rightarrow \mathcal{H}\left(i^{\prime}\right)$ is not degenerate for some $i^{\prime} \gg i$.

Question 1 (Delzant) Let $G$ be a finitely generated group that doesn't split over $\mathbb{Z}$. Are there finitely presented indecomposable covers in the category of groups with $\mathbb{Z}$ splittings? That is, is there a finitely presented group $H$ such that $H \rightarrow G$ doesn't factor through a group that splits over $\mathbb{Z}$ ? If we define "strict" to mean something like "embeds vertex and edge groups," we can imitate the above argument, replacing Scott 
complexity by something defined in terms of a "JSJ" decomposition to get an alignment theorem (see Section 6), but a uniform bound on the length of "strict resolutions" arising is still needed.

\subsection{Freely indecomposable groups}

We assume throughout this subsection that $\varphi: G \rightarrow H$ is indecomposable and that $\Delta$ is a GAD of $G$. A degenerate map either decreases the rank or first betti number, raises the Scott complexity or, barring any of these, respects Grushko decompositions. The complexity $c_{\mathrm{fd}}$ doesn't see the restrictions to freely indecomposable factors. In this subsection we use the JSJ decomposition to construct a generalization of $\boldsymbol{S}$ for freely indecomposable groups. The map $\varphi$ factors through a strict homomorphism $\boldsymbol{S}(G) \rightarrow H$, and if $\varphi$ is degenerate then $\boldsymbol{S}(G) \rightarrow H$ is an isomorphism. The explicit construction is used in the next section to show that $\operatorname{JSJ}(S(G))$ is a "degeneration" of the $\operatorname{JSJ}(G)$. In the subsections that follow we define an analogue of the Scott complexity, and give a generalization of Theorem 5.5 to JSJ decompositions.

If $T$ and $S$ are faithful simplicial $G$-trees and $\phi: T \rightarrow S$ is a $G$-equivariant morphism then $\phi$ can be realized as a composition of elementary folds $[17 ; 3 ; 6]$. As in the case of a free group, one may ignore the equivariance by looking at the quotient graphs of groups $T / G$ and $S / G$. For nonfree actions, vertex and edge stabilizers are not necessarily trivial, and an expanded set of morphisms of graphs of groups, or folds is required. All injective morphisms factor through a sequence of elementary folds, enumerated below in Figure 1.

A fold is the decorated version of a Stallings fold. Pulling has no effect on the quotient graph $T / G$, but if $\widetilde{v}$ is a lift of $v$ then the set of lifts of $e$ (as an oriented edge) adjacent to $\tilde{v}$ are in one to one correspondence with the cosets of $E$ in $V$. If $g$ is pulled across the edge then the cosets of $E$ fall into cosets of $\langle E, g\rangle$, and edges of $T$ are identified accordingly. Collapsing two parallel edges is a composition of subdivision, folding, and collapsing subgraphs.

Let $\Delta$ be a graph of groups decomposition of $G$ and $\Gamma$ a connected subgraph of $\Delta$, and let $G_{\Gamma}$ be the subgroup of $G$ carried by $\Gamma$. Collapse the graph $\Delta$ to form a graph $\Delta / \Gamma$ with distinguished vertex $\gamma$, the image of $\Gamma$. Assign the group $G_{\Gamma}$ to $\gamma$, retaining the labels on all edges and vertices not contained in $\Gamma$, and form a graph of groups decomposition $\Delta / \Gamma$ of $G$. Then $\Delta \rightarrow \Delta / \Gamma$ is a composition of collapses. If $\Gamma$ is disconnected, it is to be understood that collapsing is carried out component by component. The reverse of collapsing is blowing up.

For general maps (nonfaithful) of $G$-trees Dunwoody adds vertex morphisms to this list [6], where we pass to quotients of vertex groups. 

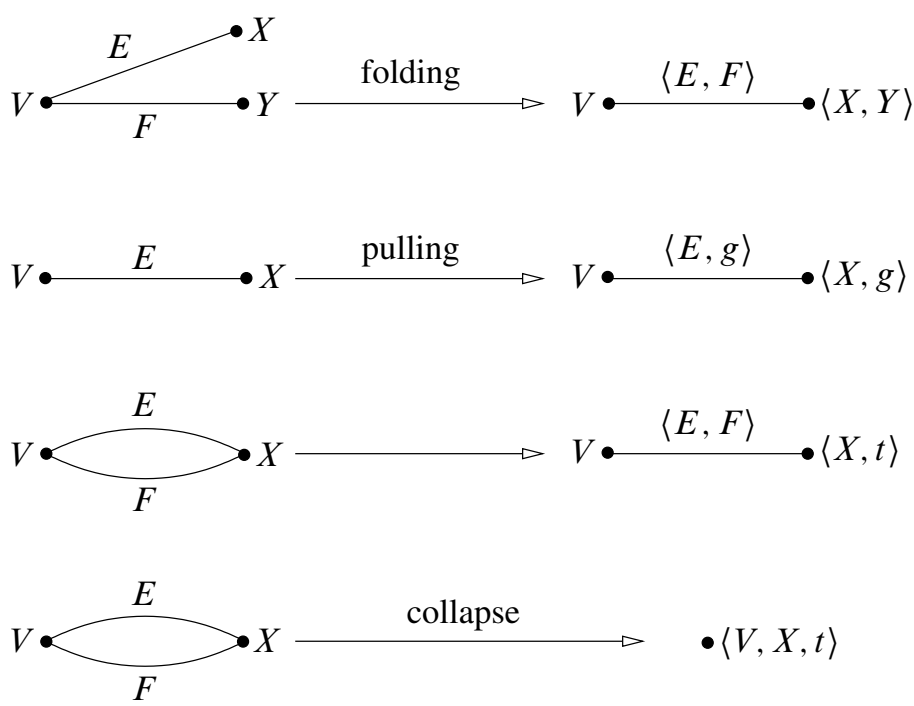

Figure 1: The basic folds (from [3], by way of [6])

5.2.1 Almost-strict homomorphisms In this section we show that if $\varphi: G \rightarrow H$ is indecomposable and $G$ is equipped with a GAD $\Delta$, then $\varphi$ factors through an almost-strict (Definition 5.7) homomorphism $\boldsymbol{A S}(G) \rightarrow H$ such that $\boldsymbol{A S}(G)$ has a GAD which resembles a degenerate, blown up $\Delta$. The construction of $\boldsymbol{A S}(G)$ is analogous to factoring a homomorphism through a group of maximal Scott complexity.

Definition 5.7 (Almost-strict) Let $\varphi: G \rightarrow H$ be indecomposable, $H$ a limit group, and $\Delta$ a GAD of $G$. If $\varphi$ satisfies the following axioms it is $\Delta$-almost-strict, or almost-strict, if $\Delta$ is understood.

- $\varphi$ embeds rigid vertex groups of $\Delta$ in $H$.

- $\varphi$ embeds edge groups of $\Delta$ in $H$.

- If $Q$ is $\mathrm{QH}$ then $\varphi(Q)$ is nonabelian.

- No rigid vertex group of $\Delta$ has a nontrivial abelian splitting in which all incident edge groups are elliptic.

- Every edge of $\Delta$ is adjacent to an abelian vertex.

This list should be compared to Theorem 2.9. Domains of almost-strict homomorphisms may not be CSA and envelopes of rigid vertex groups may not embed.

Definition 5.8 Let $G$ be a finitely generated group, and let $\mathcal{E}$ be a family of subgroups of $G$. A map $\varphi: G \rightarrow H$ is $\mathcal{E}$-indecomposable if all images of elements of $\mathcal{E}$ are nontrivial and $\varphi$ doesn't factor through a nontrivial free product in which all images of elements of $\mathcal{E}$ are elliptic. 
We will write $(G, \mathcal{E})$ for a group $G$ equipped with a family of subgroups $\mathcal{E}$. Typically $G$ will be a vertex group of a graph of groups decomposition of another group, and $\mathcal{E}$ will be the collection of images of incident edge groups.

Lemma 5.9 Suppose $G$ and $H$ are limit groups, $\varphi: G \rightarrow H$ indecomposable. If $G$ splits nontrivially over an abelian subgroup $E$ then $\varphi(E) \neq\{1\}$. If $V$ is a vertex group of a GAD of $G$ then $\left.\varphi\right|_{V}:(V, \mathcal{E}(V)) \rightarrow H$ is $\mathcal{E}(V)$-indecomposable.

Proof Let $L$ be a limit group and $E<L$ an abelian subgroup. Then $L / E$ is nontrivial. If $G$ is an amalgam $A *_{E} B$ and $\varphi(E)=\{1\}$ then $\varphi$ factors through the nontrivial free product $A / E * B / E$, contradicting indecomposability. Suppose $G=A *_{E}$, and let $E_{1}$ and $E_{2}$ be the two conjugates of $E$ contained in $A$. If $\varphi(E)=\{1\}$ then $\varphi$ factors through $\left(A /\left\langle E_{1}, E_{2}\right\rangle\right) * \mathbb{Z}$.

We first define a relative version of $\boldsymbol{S}$, which is analogous to factoring a homomorphism $\varphi:(G, \mathcal{E}) \rightarrow H$ through a pair $\left(G^{\prime}, \mathcal{E}^{\prime}\right)$ with maximal (relative) Scott complexity. See the paragraphs after Definition 5.3. We apply the relative version to the collection of vertex groups of an abelian decomposition and assemble the quotients of vertex groups into an "almost-strict factorization" of $\varphi$.

We write $A * C_{i} B_{i}$ to mean a star-shaped graph of groups with vertices $A$ and $B_{i}$, with $A$ at the center and an edge labeled $C_{i}$ connecting $A$ to $B_{i}$.

Definition 5.10 Let $G$ be a finitely generated group with a graph of groups decomposition $\Delta$. Then $c_{a}(G ; \Delta):=\sum \mathrm{b}_{1}(A / P(A))$, with the sum over all abelian vertex groups of $\Delta$.

Definition 5.11 Let $G \cong S *_{A_{i}} B_{i}, B_{i}$ free abelian, $A_{i}$ a direct summand of $B_{i}$. Let $\mathcal{E}$ be a collection of abelian subgroups of $G$. Then

$$
c_{a}(G ; \mathcal{E}):=\max _{\Delta}\left\{c_{a}(G ; \Delta) \mid E \in \mathcal{E} \Rightarrow E \text { elliptic in } \Delta\right\} .
$$

If $\varphi: G \rightarrow H, \mathcal{E}$ a family of abelian subgroups of $G$, let $\varphi(\mathcal{E}):=\{\varphi(E) \mid E \in \mathcal{E}\}$. Define

$$
c_{a}(\varphi ; \mathcal{E}):=\max _{G^{\prime}}\left\{c_{a}\left(G^{\prime} ; \varphi^{\prime}(\mathcal{E})\right) \mid \varphi \text { factors through } \varphi^{\prime}: G \rightarrow G^{\prime}\right\} .
$$

If $\varphi: G \rightarrow H$ then $G^{\prime}$ realizes $c_{a}(\varphi ; \mathcal{E})$ if $\varphi$ factors through $\varphi^{\prime}: G \rightarrow G^{\prime}$ and $c_{a}\left(G^{\prime} ; \varphi^{\prime}(\mathcal{E})\right)=c_{a}(\varphi ; \mathcal{E})$. 
Consider a map $\varphi: G \rightarrow H, G=G_{1} * \cdots * G_{p} * \mathbb{F}_{q}$, and suppose that $\boldsymbol{A S}(G)=$ $K_{1} * \cdots * K_{p^{\prime}} * \mathbb{F}_{q^{\prime}}$ is an intermediate quotient realizing $\operatorname{sc}(\varphi)$. The induced maps $K_{j} \rightarrow H$ are indecomposable. The following lemma is the analogous fact for $\mathcal{E}-$ indecomposable maps and the complexity $c_{a}$.

Lemma 5.12 Suppose $\varphi:(G, \mathcal{E}) \rightarrow H$ is $\mathcal{E}$-indecomposable, that $\varphi^{\prime}: G \rightarrow G^{\prime}$, $\psi \circ \varphi^{\prime}=\varphi$, where $\psi: G^{\prime} \rightarrow H$, and that the group $G^{\prime}=S *_{A_{i}} B_{i}$ realizes $c_{a}(\varphi ; \mathcal{E})$. Then $c_{a}\left(\left.\psi\right|_{S} ;\left\{A_{i}\right\}\right)=0$.

Let $(R, \mathcal{E}) \rightarrow H$ be an indecomposable map, and consider the collection of quotients of $R^{\prime}$ of $R$ such that $\varphi$ factors through $\pi:(R, \mathcal{E}) \rightarrow\left(R^{\prime}, \pi(\mathcal{E})\right)$, with the induced map $R^{\prime} \rightarrow H \pi(\mathcal{E})$-strict. If $R \rightarrow R_{2}$ factors through $R \rightarrow R_{1}$ then $R_{1} \geq R_{2}$, with equality if and only if $R_{1} \rightarrow R_{2}$ is an isomorphism. Choose a maximal element, which exist by Theorem 2.15, and call it $\boldsymbol{S}(R, \mathcal{E}, \varphi)$.

Definition 5.13 $S(R, \mathcal{E}, \varphi)$ is a strict intermediate quotient of $R$.

Let $\varphi:(R, \mathcal{E}) \rightarrow H$ be an $\mathcal{E}$-indecomposable map of limit groups. Choose a group $S * A_{i} B_{i}$ realizing $c_{a}(\varphi ; \mathcal{E})$. Let $\bar{A}_{i}$ be the image of $A_{i}$ in $S(S)$, and consider $\operatorname{JSJ}\left(S(S),\left\{\bar{A}_{i}\right\}\right)$. Since $c_{a}(S \rightarrow H)=0, \overline{A_{i}}$ is contained in the peripheral subgroup of the associated abelian vertex group of $\operatorname{JSJ}\left(S(S),\left\{\bar{A}_{i}\right\}\right)$, considered now as a GAD and not as a relative JSJ decomposition. In particular $\psi$ embeds $\bar{A}_{i}$. Let

$$
\mathcal{E}\left(B_{i}\right)=\left\{A_{i}, g \varphi^{\prime}(E) g^{-1} \mid g \varphi^{\prime}(E) g^{-1}<B_{i}\right\} .
$$

Set $\widehat{\boldsymbol{S}}(R):=\boldsymbol{S}\left(S,\left\{\bar{A}_{i}\right\},\left.\psi\right|_{S}\right)$ and define

$$
\boldsymbol{A S}(R, \mathcal{E}, \varphi):=\widehat{\boldsymbol{S}}(R) *_{\bar{A}_{i}} \boldsymbol{S}\left(B_{i}, \mathcal{E}\left(B_{i}\right)\right) .
$$

To save on notation we will usually write $\boldsymbol{A S}(R)$, omitting the collection $\mathcal{E}$ and the $\operatorname{map} \varphi$.

Definition 5.14 (Almost-strict intermediate quotient) The quotient $\boldsymbol{A S}(R)$ is an almost-strict intermediate quotient of $R$.

The construction of $\boldsymbol{A S}(R)$ is not canonical, and, in general $\boldsymbol{A S}(R)$ might not even be a limit group. If $c_{a}(\varphi ; \mathcal{E})=0$ then $A_{i}=B_{i}$ ( $A_{i}$ is closed under taking roots). In this case $\boldsymbol{S}(R, \mathcal{E}, \varphi)$ and $\boldsymbol{A S}(R, \mathcal{E}, \varphi)$ agree.

Let $G$ be a group with an abelian decomposition $\Delta$ and an indecomposable map $\varphi: G \rightarrow H, H$ a limit group. Build $\boldsymbol{A S}(G)$ with a splitting $\boldsymbol{A S}(\Delta)$ by passing to the following quotients of vertex groups of $G$ : For each edge group of $\Delta$, pass to 
the image in $H$. If $V$ is a vertex group with abelian image, or is a $\mathrm{QH}$ subgroup, pass to $S(V, \mathcal{E}(V))$. For each rigid vertex group of $\Delta$ with nonabelian image pass to $\boldsymbol{A S}(R, \mathcal{E}(R))$.

The gluing data from $\Delta$ descends to gluing data on this new collection of groups. Call the resulting group $\boldsymbol{A S}(G)$. For each rigid vertex group $R_{i}$ such that $\varphi\left(R_{i}\right)$ is nonabelian, $\boldsymbol{A S}\left(R_{i}, \mathcal{E}\left(R_{i}\right)\right)$ has the form $\widehat{\boldsymbol{S}}\left(R_{i}\right) * \bar{A}_{i, j} \boldsymbol{S}\left(B_{i, j}\right)$ and for each element $E \in \mathcal{E}\left(R_{i}\right)$, the image of $E$ maps to some $B_{i, j}$. Replace the vertex $A S\left(R_{i}\right)$ in $\Delta$ by the decomposition $\widehat{\boldsymbol{S}}\left(R_{i}\right) * \bar{A}_{i, j} \boldsymbol{S}\left(B_{i, j}\right)$ and for each $i$ refine the splitting on $\boldsymbol{A S}\left(R_{i}\right)$ with the normalized relative JSJ decomposition $\operatorname{JSJ}\left(\widehat{\boldsymbol{S}}(R),\left\{\bar{A}_{i, j}\right\}\right)$, and call the resulting decomposition $\boldsymbol{A S}(\Delta)$. Every nonabelian vertex group of $\boldsymbol{A S}(\Delta)$ is either a nonabelian vertex group from some $\operatorname{JSJ}\left(\widehat{\boldsymbol{S}}(R),\left\{\bar{A}_{i, j}\right\}\right)$, or a QH vertex group inherited from $\Delta$. Call the induced homomorphism of $A S(G) \rightarrow H A S(\varphi)$. To reduce the amount of notation used we simply call $\operatorname{JSJ}\left(\widehat{\boldsymbol{S}}(R),\left\{\bar{A}_{i, j}\right\}\right) \operatorname{JSJ}(\widehat{\boldsymbol{S}}(R))$.

Definition 5.15 (Almost-strict factorization) The group $\boldsymbol{A S}(G)$ with GAD $\boldsymbol{A S}(\Delta)$ and homomorphism $\boldsymbol{A S}(\varphi)$ from above is an almost-strict factorization of $\varphi$.

If $\boldsymbol{A S}(\varphi)$ isn't strict, then either it doesn't embed envelopes of rigid vertex groups or the second bullet of Theorem 2.9 doesn't hold.

Definition 5.16 If $G$ is a group then the abelianization of $G$ modulo torsion is denoted by $G^{\mathrm{ab}}$. If $\mathcal{E}$ is a collection of subgroups of $G$ then the smallest subgroup of $G^{\mathrm{ab}}$ closed under taking roots and containing the images of $\mathcal{E}$ is the peripheral subgroup of $G^{\mathrm{ab}}$ and is denoted by $P\left(G^{\mathrm{ab}}\right)$.

Lemma 5.17 Let $\varphi: G \rightarrow H$ be indecomposable, $G$ a limit group, $\Delta$ a GAD of $G$, and $G \rightarrow \boldsymbol{A S}(G)$, an $\boldsymbol{A S}(\Delta)$-almost-strict factorization of $\varphi$ as above. If $V$ is a vertex of $\Delta$ with abelian image then

$$
\boldsymbol{S}(V) \cong V^{\mathrm{ab}} / \operatorname{Ker}\left(P\left(V^{\mathrm{ab}}\right) \rightarrow H\right)
$$

and if $V$ is a $\mathrm{QH}$ subgroup with abelian image then $V$ is a punctured sphere or projective plane.

Proof The first part of the lemma is obvious. If $V$ is $\mathrm{QH}$ and has abelian image then any simple closed curve cutting off a handle has trivial image and $\varphi$ would have factored through a free product $G^{\prime} * \mathbb{Z}^{2}$.

Lemma 5.18 Let $\varphi: G \rightarrow H$ be indecomposable, $G$ a limit group, $\Delta$ a GAD of $G$, and $G \rightarrow \boldsymbol{A S}(G)$, an $\boldsymbol{A S}(\Delta)$-almost-strict factorization of $\varphi$ as above. 
Suppose that $\varphi(V)$ is abelian, and that $V$ has a graph of groups decomposition $V \cong$ $\Gamma\left(A_{i}, F_{j}\right)$ over torsion free abelian vertex groups $A_{i}$ and nontrivial edge groups $F_{j}$, with each edge group of $\Delta$ incident to $V$ elliptic in $\Gamma$. Let $P\left(A_{i}\right)$ be the subgroup of $A_{i}$ generated by incident edge groups (in $\Gamma$ ) and those elements of $\mathcal{E}(V)$ conjugate into $A_{i}$. Call the underlying graph of $\Gamma\left(A_{i}, F_{j}\right) \Gamma$ as well. Then

$$
\sum_{i} \operatorname{rk}\left(A_{i} / P\left(A_{i}\right)\right)+\mathrm{b}_{1}(\Gamma) \leq \operatorname{rk}(S(V) /\langle\operatorname{Im}(\mathcal{E}(V))\rangle) .
$$

Proof Indecomposability of $\varphi$ and Lemma 5.9 imply that $F_{j}$ has nontrivial image in $H$. Construct an abelian quotient $\bar{V}$ of $V$ as follows: Let $T$ be a maximal tree in $\Gamma$ and let $F_{i_{1}}, \cdots, F_{i_{m}}$ be the edge groups of $\Gamma$ not carried by edges in $T$. Let $V_{T}$ be the subgraph of groups of $V$ obtained by restriction to $T$, and let $\mathcal{E}\left(V_{T}\right)$ be the collection $\mathcal{E}(V)$ along with the $F_{i_{j}}$. The inequality

$$
\sum_{i} \operatorname{rk}\left(A_{i} / P\left(A_{i}\right)\right) \leq \operatorname{rk}\left(S\left(V_{T}\right) /\left\langle\operatorname{Im}\left(\mathcal{E}(V) \cup\left\{F_{i_{j}}\right\}\right)\right\rangle\right)
$$

holds. Let $t_{j}$ be the stable letter associated to $F_{i_{j}}$. Then the image of $t_{j}$ in $H$ conjugates the image of $F_{i_{j}}$ to another subgroup of the image of $P\left(S\left(V_{T}\right)\right)$. The two inclusions $F_{i_{j}} \rightarrow S\left(V_{T}\right)$ are both nontrivial, since $\varphi$ is indecomposable, and since limit groups are CSA, they agree with each other, hence $V_{T} * F_{i_{j}} \rightarrow H$ factors through $V_{T} \oplus\left\langle t_{j}\right\rangle$, which satisfies the inequality of the lemma. Repeating over all edge groups $F_{i_{j}}$ we find an abelian quotient $V^{\prime}$ of $V$, a $\operatorname{Im}(\mathcal{E}(V))$-strict $V^{\prime} \rightarrow H$ satisfying the lemma. Since $V^{\prime} \rightarrow H$ factors through $\boldsymbol{S}(V) \rightarrow H, V^{\prime} \cong \boldsymbol{S}(V)$ by maximality.

5.2.2 Almost-strict to strict Fix a $\Delta$-almost-strict $\varphi: G \rightarrow H$. We define maps $\boldsymbol{S}_{\mathrm{A}}$ and $\boldsymbol{S}_{\mathrm{E}}$ taking as input $\varphi$ and output $\boldsymbol{S}_{*}(\Delta)$-almost-strict maps $\boldsymbol{S}_{*}(\varphi): \boldsymbol{S}_{*}(G) \rightarrow H$ which are closer to satisfying Theorem 2.9. The decompositions $\boldsymbol{S}_{*}(\Delta)$ are modeled on $\Delta$, but differ by a sequence of blow-ups, blow-downs, and folds. We then iterate, producing a sequence $\left(\boldsymbol{S}_{\mathrm{A}} \circ \boldsymbol{S}_{\mathrm{E}}\right)^{i} \boldsymbol{A} \boldsymbol{S}(G)$ which converges to a limit quotient $\boldsymbol{S}(G)$ of $G, \varphi$ factors through $\boldsymbol{S}(G)$, and the induced map $\boldsymbol{S}(\varphi): \boldsymbol{S}(G) \rightarrow H$ is strict.

Adjust the decomposition $\Delta$. Let $\sim_{a}$ be the equivalence relation generated by adjacency of abelian vertex groups. Let $[A]$ be a $\sim_{a}$ equivalence class and let $\Gamma_{[A]}$ be the subgraph of $\Delta$ with vertices from $[A]$ and edges connecting members of $[A]$. Collapse the subgraphs $\Gamma_{[A]}$ and call the vertex associated to $[A] v_{[A]}$.

Let $S_{\mathrm{A}}(G)$ be the group obtained from $G$ by passing from $G_{v_{[A]}}$ to $S\left(G_{v_{[A]}}\right)$ for each $\sim_{a}$ equivalence class $\mathcal{A}$. The splitting $\boldsymbol{S}_{\mathrm{A}}(\Delta)$ of $\boldsymbol{S}_{\mathrm{A}}(G)$ is the push forward of $\Delta$. The induced map $\boldsymbol{S}_{\mathrm{A}}(G) \rightarrow H$ is denoted $\boldsymbol{S}_{\mathrm{A}}(\varphi)$. 
The induced map of the quotient group $\boldsymbol{S}_{\mathrm{A}}(G)$ with the GAD it inherits from $G$ is almost-strict. We now define $\boldsymbol{S}_{\mathrm{E}}$, which brings envelopes of rigid vertex groups closer to embedding.

Definition 5.19 Let $R_{1}$ be a limit group, $\mathcal{E}$ a family of nonconjugate abelian subgroups of $R_{1}$ such that the relative $\mathrm{JSJ}$ decomposition $\operatorname{JSJ}\left(R_{1} ; \mathcal{E}\right)$ is trivial. An envelope of $R_{1}$ is a limit group $R_{2}$ such that:

- There is a collection $\left\{P_{i}\right\}_{i \in I}$ of free abelian groups such that $R_{2}$ is a limit quotient of $R_{1} * E_{i} P_{i}$,

- There is a map from $R_{1} * E_{i} P_{i}$ to a fixed limit group $H$.

- $R_{1} \rightarrow R_{2}$ is injective and the map $R_{1} * E_{i} P_{i} \rightarrow H$ factors through the map to $R_{2}$.

- The map from $R_{2}$ to $H$ is $\left\{\operatorname{Im}\left(P_{i}\right)\right\}$-strict.

We call the group $R_{1} * E_{i} P_{i}$ a pre-envelope. We call $R_{1}$ the core of the pre-envelope. The complexity of a pre-envelope is the ordered tuple

$$
\left(\sum_{i} \operatorname{rk}\left(P_{i} / E_{i}\right),|I|, r, m\right),
$$

where $r$ is the number of indices such that $E_{i}$ is not closed under taking roots in $P_{i}$, and $m$ is the number of indices such that $E_{i}$ is not maximal abelian in $R_{1}$.

Envelopes can be written as the output of a process of iteratively adjoining roots and extension of centralizers.

Definition 5.20 (Iteratively adjoining roots; see also Definition 9.1) Let $L$ be a limit group. Then $L^{\prime}$ is obtained from $L$ by iteratively adjoining roots if there is a finite sequence of limit groups $L_{i}, L_{0}=L, L_{n}=L^{\prime}$ such that:

- $L_{i+1}$ is obtained from $L_{i}$ by adjoining roots to the collection $\mathcal{E}_{i}$ : for each $E \in \mathcal{E}_{i}$ there is an abelian group $F(E)$, a finite index inclusion $E \hookrightarrow F(E)$, and the inclusion map $L_{i} \hookrightarrow L_{i+1}$ extends to $L_{i} *_{E \in \mathcal{E}_{i}} F(E)$.

- If $E \in \mathcal{E}_{i}$ then there is an $F \in \mathcal{E}_{i-1}$ such that $E=Z_{L_{i}}(\operatorname{Im}(F))$.

Lemma 5.21 Let $R_{2}$ be an envelope of $R_{1}$, with $\operatorname{Mod}\left(R_{2} ;\left\{\operatorname{Im}\left(P_{i}\right)\right\}\right)$ strict homomorphism $\varphi: R_{2} \rightarrow H$ which embeds $R_{1}$. Then $R_{2}$ is obtained as an iterated adjunction of roots and an extension of centralizers, and can be realized as a quotient pre-envelope of the pre-envelope $R_{1} * E_{i} P_{i}$ of lower complexity. 
Let $\left\{\mathcal{F}_{j}\right\}$ be the set of equivalence classes of $E_{i}$ in $R_{2}$ such that $E_{i}, E_{i^{\prime}} \in \mathcal{F}_{j}$ if and only if $E_{i}$ and $E_{i^{\prime}}$ have conjugate centralizers. For each class $\mathcal{F}_{j}$ choose a single representative element $F_{j}$, and for $j$ let $I_{j}$ be the set of indices $i$ such that $E_{i} \in \mathcal{F}_{j}$. Then there are direct sum decompositions $P_{i} \cong P_{i}^{\prime} \oplus C_{i}$ such that $E_{i}<C_{i}$, quotients $D_{j}$ of $\bigoplus_{i \in I_{j}} P_{i}^{\prime}$, and $R_{2}$ can be written as

$$
\bar{R}_{1}:=R_{1}\left[\sqrt{E}_{i}\right], \quad R_{2} \cong \bar{R}_{1} * Z_{\bar{R}_{1}}\left(F_{j}\right)\left(Z_{\bar{R}_{1}}\left(F_{j}\right) \oplus D_{j}\right) .
$$

The group $\bar{R}_{1}$ is a quotient of $R_{1} * E_{i} C_{i}$, has trivial JSJ relative to the centralizers of the images of the $E_{i}$, and $\varphi$ is an embedding.

Lemma 5.22 Let $H$ be a limit group, $L$ a pre-envelope $G * A_{i} B_{i}, A_{i}$ maximal abelian in $G$, closed under taking roots in $B_{i}$, and $A_{i}$ not conjugate to $A_{j}$ for $i \neq j$ in $G$. Let $\varphi: L \rightarrow H$ be a homomorphism such that all restrictions $\left.\varphi\right|_{B_{i}}$ and $\left.\varphi\right|_{G}$ are injective. Then $\varphi$ is injective.

The argument is standard, and follows from normal forms, induction on the depth of the analysis lattice ${ }^{3}$, and the fact that if two elements of a group are nonconjugate then they remain nonconjugate after extension of centralizers.

Proof of Lemma 5.21 We define three types of intermediate quotients of pre-envelopes. Each homomorphism either yields a pre-envelope of lower complexity or the map to $H$ is an embedding.

Suppose $E_{i}$ is not maximal abelian in $R_{1}$. Let $C_{i}$ be the smallest direct summand of $P_{i}$ containing $E_{i}$, and let $D_{i}$ be a complimentary direct summand. By commutative transitivity the map $R_{1} * E_{i} P_{i} \rightarrow R_{2}$ factors through

$$
P_{j \neq i} * E_{j} R_{1} * Z_{R_{1}}\left(E_{i}\right)\left(\left(Z_{R_{1}}\left(E_{i}\right) * E_{i} C_{i}\right)^{\mathrm{ab}} \oplus D_{i}\right) .
$$

The last coordinate of the complexity decreases.

If, without loss, $E_{1}$ and $E_{2}$ have conjugate centralizers, then the map $R_{1} *_{E_{i}} P_{i} \rightarrow H$ factors through

$$
R_{1}^{\prime}:=P_{j>2} * E_{j>2} R_{1} * Z_{R_{1}}\left(E_{1}\right)\left(Z_{R_{1}}\left(E_{1}\right) \oplus D_{1} \oplus D_{2}\right) .
$$

In this case the pre-envelope $R_{1}^{\prime}$ has lower complexity than $R_{1}$, since second coordinate of the complexity strictly decreases.

The third quotient of a pre-envelope is taken only if neither of the first two can be: Let $C_{i}$ be the largest direct summand of $P_{i}$ such that the image of $E_{i}$ has finite index

\footnotetext{
${ }^{3}$ Define, in analogy with the principal cyclic analysis lattice, the abelian analysis lattice, given by passing to vertex groups of the abelian JSJ at each level.
} 
image in the image of $C_{i}$, let $D_{i}$ be a complimentary direct summand, and let $\bar{C}_{i}$ be the image of $C_{i}$ in $R_{2}$. Let $R_{1}^{\prime}$ be the image of $R_{1} * E_{i} * \bar{C}_{i}$ in $R_{2}$. Since $E_{i}$ is finite index in $\bar{C}_{i}$, the relative JSJ decomposition of $R_{1}^{\prime}$ is also trivial and $R_{1}^{\prime}$ must embed in $R_{2}$ and $H$.

Then $R_{2}$ is a limit quotient of a new pre-envelope

$$
R_{1}^{\prime \prime}:=R_{1}^{\prime} * \bar{C}_{i}\left(\bar{C}_{i} \oplus D_{i}\right)
$$

Since $C_{i}$ contains $E_{i}$, the complexity of $R_{1}^{\prime \prime}$ is at most that of $R_{1}^{\prime} *_{E_{i}} P_{i}$, and the core $R_{1}^{\prime}$ has trivial JSJ relative to $\bar{C}_{i}$ follows from the fact that $R_{1}^{\prime}$ is obtained from $R_{1}$ by adjoining roots. If the third coordinate of the complexity cannot be decreased by passing to the pre-envelope $R_{1}^{\prime \prime}$ then $C_{i}=E_{i}$, each $P_{i}$ must embed in $R_{2}$, and $E_{i}$ also be maximal abelian in $R_{1}$. By Lemma 5.22, $R_{2}=R_{1} * E_{i} P_{i} \rightarrow H$ is an embedding.

Let $\varphi: G \rightarrow H$ be $\Delta$-almost-strict. Then $\varphi$ might not embed envelopes of rigid vertex groups. First adjust $\Delta$ : choose an edge incident to a rigid vertex group $R$, and let $A$ be the abelian vertex group attached to the other end of the edge. Subdivide, pull $P(A)$ across the new edge, and repeat for all edges incident to rigid vertex groups, and call the new abelian decomposition $\Delta^{\prime}$. For an edge group $E$, let $A(E)$ be the conjugate of the abelian vertex group adjacent to $E$. For each rigid vertex group of $\Delta^{\prime}$, the star of $R$ now has the form

$$
\operatorname{St}(R) \cong R *_{E \in \mathcal{E}(R)} P(A(E)) .
$$

Now replace $\operatorname{St}(R)$ by $\boldsymbol{A} \boldsymbol{S}\left(\operatorname{St}(R),\left\{P\left(A_{i}\right)\right\}\right)$. By Lemma 5.21, since $R$ embeds in $H$ and has no splittings relative to $\mathcal{E}(R)$,

$$
S\left(\operatorname{St}(R),\left\{P\left(A_{i}\right)\right\}\right) \cong \bar{R} * Z\left(F_{j}\right)\left(C\left(F_{j}\right) \oplus D_{j}\right) .
$$

Each peripheral subgroup $P\left(A_{i}\right)$ has image contained in some $C\left(F_{j}\right) \oplus D_{j}$. Since $P\left(A_{i}\right)$ embeds in $H$ it embeds in $C\left(F_{j}\right) \oplus D_{j}$. Repeat for all rigid vertex groups of $\Delta^{\prime}$ and call the quotient group $\boldsymbol{S}_{\mathrm{E}}(G)$, the induced decomposition $\boldsymbol{S}_{\mathrm{E}}(\Delta)$ (the pushforward of $\Delta$ ), and the induced homomorphism $\boldsymbol{S}_{\mathrm{E}}(\varphi)$. The induced map $\boldsymbol{S}_{\mathrm{E}}(\varphi): \boldsymbol{S}_{\mathrm{E}}(G) \rightarrow H$ is clearly $\boldsymbol{S}_{\mathrm{E}}(\Delta)$-almost-strict.

We can now define the direct limit of almost-strict intermediate quotients. Let $G \rightarrow H$ be an indecomposable homomorphism of limit groups. Let $G_{i}$ be the group

$$
G_{0}=\boldsymbol{A S}(G), \quad G_{2 n+1}=\boldsymbol{S}_{\mathrm{A}}\left(G_{2 n}\right), \quad G_{2 n}=\boldsymbol{S}_{\mathrm{E}}\left(G_{2 n-1}\right) .
$$

Let $\Delta_{2 n+1}$ and $\Delta_{2 n+2}$ be the associated pushforwards $S_{\mathrm{A}}\left(\Delta_{2 n}\right)$ and $\boldsymbol{S}_{\mathrm{A}}\left(\Delta_{2 n-1}\right)$. Let $G_{\infty}$ be the direct limit of the sequence $\left(G_{n}\right)$. The map $G \rightarrow H$ factors through $G_{\infty}$. 
Theorem 5.23 The direct limit $G_{\infty}$ is a limit group and $G_{n} \rightarrow G_{n+1}$ is an isomorphism for all but finitely many $n$. For sufficiently large $n$ the decompositions $S_{\mathrm{A}}\left(\Delta_{2 n}\right)$ and $\boldsymbol{S}_{\mathrm{E}}\left(\Delta_{2 n-1}\right)$ agree up to pulling subgroups of abelian vertex groups. The rigid vertex groups in the decomposition of $G_{\infty}$ induced by $A S(\Delta)$ are obtained from the rigid vertex groups of $\boldsymbol{A S}(\Delta)$ by iteratively adjoining roots.

Proof of Theorem 5.23 By Lemma $5.18 c_{a}\left(G_{n}, \Delta_{n}\right)$ (Definition 5.11) is nondecreasing. Since $\mathrm{b}_{1}\left(G_{n}\right) \leq \mathrm{b}_{1}(G)$ we may drop finitely many terms from the beginning of the sequence and assume that the sequence $c_{a}\left(G_{n}, \Delta_{n}\right)$ is constant. If this is the case then the underlying graphs of the $\Delta_{i}$ have constant first betti number. Let $\left\{R_{j}^{i}\right\}$ be the rigid vertex groups of $\Delta_{i}$. By Lemma $5.21 R_{j}^{i+1}$ is obtained from $R_{j}^{i}$ by iteratively adjoining roots. Let $v_{j}^{i}$ be the number of edges incident to $R_{j}^{i}$. The sequence $\left(v_{j}^{i}\right)_{i=1, \ldots, \infty}$ is nonincreasing. Again, by dropping finitely many terms from the beginning of the sequence, we may assume that all the sequences $v_{j}^{i}$ are constant. Once this is the case, the sequence of numbers of conjugacy classes of abelian vertex groups of $G_{2 n+1}$ is constant.

Let $A_{k}^{2 n+1}$ be the collection of sequences of abelian vertex groups, $A_{k}^{2 n+1}<G_{2 n+1}$ such that $A_{k}^{2 n+1} \rightarrow A_{k}^{2 n+3}$ for all $n$. Inside $\Delta_{2 n+1}$ there are subgraphs of groups of the form

$$
R_{i(l)}^{2 n+1} \frac{}{E_{l}^{2 n+1}} A_{k(l)}^{2 n+1} .
$$

In passing to $G_{2 n+2}$, by subdividing and pulling, subgraphs of this form are transformed to subgraphs of groups of the form

$$
R_{i(l)}^{2 n+1} E_{E_{l}^{2 n+1}} P\left(A_{k(l)}^{2 n+1}\right) \frac{}{P\left(A_{k(l)}^{2 n+1}\right)} A_{k(l)}^{2 n+1},
$$

which, after an application of Lemma 5.21, become the subgroups

$$
R_{i(l)}^{2 n+2} \longrightarrow{ }_{\mathcal{Z}\left(E_{l}^{2 n+1}\right)} \mathcal{Z}\left(E_{l}^{2 n+1}\right) \oplus D_{l}^{2 n+2} \frac{}{P\left(A_{k(l)}^{2 n+1}\right)} A_{k(l)}^{2 n+1}
$$

contained in $G_{2 n+2}$. The centralizer $\mathcal{Z}\left(E_{l}^{2 n+1}\right)$ should be taken in $R_{i(l)}^{2 n+2}$. Now to pass to $G_{2 n+3}$, the edges that are labeled $P\left(A_{k}^{2 n+1}\right)$ are crushed when collapsing the subtrees (they are trees since $c_{a}$ is constant) $\Gamma_{[A]} \subset \Delta_{2 n+1}$. Since

$$
E_{l}^{2 n+1} \hookrightarrow P\left(A_{k(l)}^{2 n+1}\right)
$$

and $P\left(A_{k(l)}^{2 n+1}\right)$ is generated by incident edge groups, and since

$$
E_{l}^{2 n+1}<\mathcal{Z}_{R_{i(l)}^{2 n+2}}\left(E_{l}^{2 n+1}\right) \cong E_{l}^{2 n+2},
$$


we have $P\left(A_{k(l)}^{2 n+1}\right)<P\left(A_{k(l)}^{2 n+3}\right)$. Since $P\left(A_{k(l)}^{2 n+1}\right)$ embeds in $H$ by construction, the sequences

$$
P\left(A_{k(l)}^{2 n+1}\right)<P\left(A_{k(l)}^{2 n+3}\right)
$$

map to ascending chains of subgroups of a finitely generated free abelian subgroup of $H$, and therefore for sufficiently large $n, P\left(A_{k}^{2 n+1}\right)=P\left(A_{k}^{2 n+3}\right)$. Likewise, the sequences

$$
\mathcal{Z}_{R_{i(l)}^{2 n+2}}\left(E_{l}^{2 n+1}\right)
$$

eventually stabilize hence $R_{i}^{2 n+2} \rightarrow R_{i}^{2 n+4}$ is an isomorphism for all $i$ and sufficiently large $n$.

Let $n$ be large enough to satisfy the above. If $E$ is adjacent to $R$ and $E$ doesn't have maximal abelian image in $R$ then $\boldsymbol{S}_{\mathrm{A}} \circ \boldsymbol{S}_{\mathrm{E}}$ strictly increases the rank of some peripheral subgroup, contradicting the stability of ranks of peripheral subgroups (Recall the normalization that every edge group be adjacent to a maximal abelian vertex group.). If the envelope of a rigid vertex group doesn't embed, then the either the rank of an edge group must increase under $S_{\mathrm{E}}$ or a peripheral subgroup must fail to embed, neither of which is possible. By Lemma 5.22 the envelopes must embed. By Theorem 2.9, $G_{n} \rightarrow H$ is strict for sufficiently large $n$.

That the rigid vertex groups are obtained by iteratively adjoining roots to vertex groups of $\boldsymbol{S}(G)$ follows from the fact that rigid vertex groups of $\operatorname{JSJ}(\widehat{\boldsymbol{S}}(R))$ have no splittings relative to their incident edge groups, the fact that peripheral subgroups stabilize, and Lemma 5.21.

Set $\boldsymbol{S}(G):=G_{\infty}$, likewise for $\boldsymbol{S}(\Delta)$ and $\boldsymbol{S}(\varphi)$. We say that $\boldsymbol{S}(\Delta)$ is the pushforward of $\Delta$.

\section{Degenerations of JSJ decompositions}

We saw in the previous section that an indecomposable $\varphi: G \rightarrow H$ factors through a quotient $\boldsymbol{S}(G)$ of $G$, and that the induced map $\boldsymbol{S}(\varphi): S(G) \rightarrow H$ is strict. Our approach to Theorem 1.1 is to use Theorems 5.4 and 4.3 to reduce the problem of existence of arbitrarily long chains of epimorphisms of limit groups to an analysis of indecomposable degenerate chains. A chain $\mathcal{L}$ is indecomposable if all maps $\mathcal{L}(i) \rightarrow \mathcal{L}(j), i<j$, are indecomposable.

Consider an indecomposable map $\varphi: G \rightarrow H$. The map $\boldsymbol{S}(\varphi)$ is induced by a (finite) direct limit of quotients of $G$ obtained by repeatedly applying $\boldsymbol{S}_{\mathrm{A}}$ and $\boldsymbol{S}_{\mathrm{E}}$. These homomorphisms were designed to ensure that in the limit the bullets from Theorem 2.9 
were satisfied. The possible degenerations of the abelian JSJ of $G$ under $\boldsymbol{S}$ were not completely characterized since we were only interested in constructing a strict factorization. Now we keep track of how the abelian JSJ of $\boldsymbol{S}(G)$ is obtained from the abelian JSJ of $G$.

We define a complexity of a limit group using the abelian JSJ decomposition with three types of terms: counting $\mathrm{b}_{1}$ naively, complexity of $\mathrm{QH}$ vertex groups not reflected in the first betti number, and some combinatorics of the underlying graph. The complexity is nondecreasing on degenerate indecomposable chains, and the number of values it takes is controlled by the first betti number.

Define, for an abelian decomposition $\Delta$ :

- The sum of relative first betti numbers of abelian vertex groups:

$$
c_{a}(G, \Delta):=\sum_{A} \mathrm{~b}_{1}(A / P(A))
$$

- The complexity of surface vertex groups: If $\Sigma$ is a surface with boundary then

$$
c_{g}(G, \Delta):=\sum_{\Sigma(Q)} \mathrm{b}_{1}(\Sigma, \partial \Sigma)
$$

with the sum over all surfaces $\Sigma(Q)$ representing QH vertex groups $Q$ of $G$.

- Let $\Gamma$ be the underlying graph of $\Delta$ : The betti number of the underlying graph of $\Delta$ :

$$
c_{b}(G, \Delta):=\mathrm{b}_{1}(\Gamma)
$$

The complexity of a limit group is, to a first approximation,

$$
\operatorname{sc}_{1}(G, \Delta):=\left(c_{a}(G, \Delta), c_{g}(G, \Delta), c_{b}(G, \Delta)\right) .
$$

If " $\Delta$ " is omitted then the abelian JSJ decomposition is implied. By Lemma 5.18 the first coordinate of $\mathrm{sc}_{1}$ is nondecreasing under indecomposable degenerate maps. Lemma 6.2 is the analog of Lemma 5.18 for surfaces with boundary.

Lemma 6.1 Let $\Sigma$ be a surface with boundary, written as a graph of surfaces with boundary $\Gamma\left(\Sigma_{i}\right)$. Then

$$
\mathrm{b}_{1}(\Sigma, \partial \Sigma) \geq \mathrm{b}_{1}(\Gamma)+\sum_{i} \mathrm{~b}_{1}\left(\Sigma_{i}, \partial \Sigma_{i}\right)
$$

Proof There is a map $\Sigma \rightarrow \mathbb{Z}^{\mathrm{b}_{1}(\Gamma)}$ which kills $\partial \Sigma$ and all $\Sigma_{i}$.

Lemma 6.2 Suppose $c_{a}(G)=c_{a}(H)$. Then $c_{g}(G) \leq c_{g}(H)$. If equality holds then $c_{b}(G) \leq c_{b}(H)$. 
Proof Let $\Delta=\boldsymbol{S}(\operatorname{JSJ}(G))$ be the pushforward of the abelian JSJ of $G$ under $\boldsymbol{S}$. Since $G \rightarrow H$ is degenerate, the map $S(G) \rightarrow H$ is an isomorphism. Thus, we only need to deduce how the JSJ of $H$ is obtained from $\Delta$.

If $\Delta$ is a GAD of $H$ and $H$ has an abelian JSJ decomposition, then $\Delta$ can be obtained (up to folding, sliding, and collapsing) from the JSJ by choosing a family of simple closed curves $c_{i}$ on $\mathrm{QH}$ vertex groups, regarding the resulting subsurfaces as vertex groups and the curves $c_{i}$ as edge groups, and then folding and collapsing subgraphs, therefore if $R$ is a rigid vertex group of $\Delta$ then either $R$ is a rigid vertex group in the JSJ of $H$, or $R$ is represented by a non-QH subsurface of a QH vertex group of $H$. Such a subsurface group must be either a twice punctured projective plane or a thrice punctured sphere.

Every rigid vertex group $R$ of $\Delta$ is either a subsurface or a rigid vertex group from the JSJ of $H$. If $R$ is a subsurface then, since $R$ isn't a QH vertex group of the relative abelian JSJ of $R$, it must be one of the two non-QH surface groups above. We recover the abelian JSJ of $H$ by gluing the QH vertex groups of $\Delta$ and the "rigid" subsurface groups along their boundary components. Define, in analogy with $\sim_{a}$, an equivalence relation $\sim_{q}$ on subsurface groups $R, \mathrm{QH}$ vertex groups $Q$, and cyclic vertex groups $Z$ : If $V$ is a cyclic abelian vertex group of valence two, regard it as the fundamental group of an annulus, and if valence one, of a Möbius band. If two such groups $V_{1}$ and $V_{2}$ are adjacent, being the endpoints of an edge $e$, then $V_{1} \sim_{q} V_{2}$ if the inclusions $E \hookrightarrow V_{i}$ are isomorphisms with boundary components. As for $\sim_{a}$ equivalence classes $[A]$, let $\Gamma_{\mathcal{Q}}$ be the subgraph spanned by edges connecting vertices of a $\sim_{q}$ equivalence class $\mathcal{Q}$.

If a cycle $C$ of such vertex groups appears in $\Gamma_{Q}$, then by Lemma 6.1,

$$
c_{g}(H, \Delta / C)+c_{b}(H, \Delta / C) \geq c_{g}(H, \Delta)+c_{b}(H, \Delta) .
$$

Collapsing all such cycles, we see that $c_{g}(G)+c_{b}(G) \leq c_{g}(H, \Delta)+c_{b}(H, \Delta) \leq$ $c_{g}(H)+c_{b}(H)$. If equality holds then no cycles are collapsed, and if a cycle is collapsed then the inequality is strict.

We now add terms to $\mathrm{sc}_{1}$ which handle the possibility that nonabelian vertex groups may have abelian image under degenerate maps. For the remainder of the section, $G \rightarrow H$ is a degenerate map such that $\operatorname{sc}_{1}(G)=\operatorname{sc}_{1}(H)$. If $R$ is a valence one vertex group, then, since the first betti number relative to an abelian subgroup is at least one, $R$ cannot have abelian image in $H$, otherwise $c_{a}(H)>c_{a}(G)$. Likewise, the underlying graph of the JSJ of any $\boldsymbol{S}(R)$, for a rigid vertex group $R$ of $G$, cannot contain a loop (which makes a contribution to $c_{b}$ ) or, through abelian vertex groups in their relative JSJ's, make any contributions to $c_{a}$, in other words, they must be trees 
with abelian subgroups equal to their peripheral subgroups. Let $\Gamma_{\mathcal{A}}$ be a subgraph of the underlying graph of the JSJ of $G$ such that $G_{\Gamma_{\mathcal{A}}}$ has abelian image in $H$. If $c_{a}(G)=c_{a}(H)$ then $\Gamma_{\mathcal{A}}$ must be a tree. If $G_{\Gamma_{\mathcal{A}} * E_{i}} G^{\prime}$ is the splitting of $G$ obtained by collapsing all edges of $G$ except those adjacent to $\Gamma_{\mathcal{A}}$ but not contained in $\Gamma_{\mathcal{A}}$, then no two images $\operatorname{Im}\left(E_{i}\right), \operatorname{Im}\left(E_{j}\right)$ can have conjugate centralizers in $\boldsymbol{A S}\left(G^{\prime}\right)$, otherwise $c_{a}(H)>c_{a}(G)$.

Lemma 6.3 Suppose $G \rightarrow H$ is degenerate and $\operatorname{sc}_{1}(G)=\operatorname{sc}_{1}(H)$. Let $R$ be a nonabelian vertex group of $G$ (either rigid or $\mathrm{QH}$ ) with abelian image in $H$. Let $e_{1}, \ldots, e_{n}$ be the edges of $G$ such that $e_{i}$ doesn't connect $r$, the vertex carrying $R$, to a valence one abelian vertex group of $G$. Then the collection of images of $E_{i},\left\langle E_{i}\right\rangle<R$, generate $\mathrm{H}_{1}(R)$, and if an incident edge is separating, then neither vertex group in the corresponding one-edged splitting has abelian image in $H$. In particular, if $R$ is $\mathrm{QH}$, then at most one edge incident to $R$ connects it to a valence one abelian vertex group. In either case, the abelian vertex group of $H$ containing the image of $R$ has valence at least two.

Proof If incident edge groups don't generate $\mathrm{H}_{1}(R)$ then there is a map $R \rightarrow \mathbb{Z}$ which kills every incident edge and $c_{a}$ goes up. Suppose $Q$ is a $\mathrm{QH}$ vertex group of $G$ with abelian image in $H$, then, by indecomposability of $G \rightarrow H, Q$ has no genus and is either a punctured sphere or projective plane, and since $\chi(Q) \leq-2$ it has at least three boundary components. Suppose $Q$ has two adjacent valence one abelian vertex groups $A_{1}$ and $A_{2}$. Let $P$ be a pair of pants with boundary components $l_{1}, l_{2}$ and $w$, such that $l_{i}$ is the leg of $P$ attached to $A_{i}$. Then the group $\mathcal{A}=A_{1} * l_{1} P * l_{2} A_{2}$ satisfies $\mathrm{b}_{1}(S(\mathcal{A}, w))>\mathrm{b}_{1}\left(A_{1}, l_{1}\right)+\mathrm{b}_{1}\left(A_{2}, l_{2}\right)$, leading to a strict increase in $c_{a}$.

Since the first betti number of a nonabelian limit group relative to an abelian subgroup is at least one, $n$ is at least two. Suppose that some edge $e_{i}$ is separating, $G=G_{1} * E_{i} G_{2}$, and $R$ (or $Q$ ) is contained in $G_{1}$. If $G_{2}$ has abelian image in $H$ then the relative JSJ decomposition $\operatorname{JSJ}\left(G_{2}, E_{i}\right)$ is a tree. If $G_{2}$ contains a rigid vertex group $R^{\prime}$ of $G$ then $R^{\prime}$ isn't a valence one vertex group, is a cut-point in the underlying graph of $\operatorname{JSJ}(G)$, and has as many complimentary components as it does incident edge groups. Likewise, if $Q^{\prime}$ is a $\mathrm{QH}$ subgroup of $G$ contained in $G_{2}$, then the vertex associated to $Q^{\prime}$ must be a cut-point in the underlying graph of the JSJ and must have as many complimentary components as it does boundary components.

Thus, if $G_{2}$ contains a nonabelian vertex group of $G$, then it must contain one for which all incident edges but one are attached to valence one abelian vertex groups of $G$. This is of course an impossibility, as $c_{a}(H)$ is then strictly larger than $c_{a}(G)$. 
We continue the analysis by appending terms to $\mathrm{sc}_{1}$ which guarantee that only valencetwo rigid and abelian vertex groups can have abelian image. It is also possible for QH subgroups to grow, and for rigid vertex groups to engender multiple child vertex groups.

Recall that we assume $G \rightarrow H$ is degenerate and that $\operatorname{sc}_{1}(G)=\operatorname{sc}_{1}(H)$.

Definition 6.4 If $\Gamma$ is a finite graph, $v$ a vertex of $\Gamma$, then define

$$
\kappa(v):=1-\frac{1}{2} \operatorname{valence}(v) .
$$

By Gauss-Bonnet $\chi(\Gamma)=\sum_{v \in \Gamma^{0}} \kappa(v)$. Let $\Gamma$ be the underlying graph of an abelian decomposition $\Delta$ of a limit group $G$. Then define

$$
\begin{aligned}
\kappa_{N}(\Delta) & :=\sum_{G_{v} \text { nonabelian }} \kappa(v), \\
\kappa_{A}(\Delta) & :=\sum_{G_{v} \text { abelian }} \kappa(v) .
\end{aligned}
$$

By definition $\kappa_{A}(\Delta)+\kappa_{N}(\Delta)=\chi(\Gamma)$.

Suppose $v$ is a valence one vertex of $\Delta$. Then, regardless of whether or not $G_{v}$ is abelian, $G_{v}$ contributes at least one to $\mathrm{b}_{1}(G)$. Let $\kappa_{N \mid A}^{+}$be the total contribution of nonabelian|abelian valence one vertex groups to $\kappa_{N \mid A}$, and let $\kappa_{N \mid A}^{-}$be the total contribution of nonabelian|abelian vertex groups of valence at least three. All other vertex groups have valence two and make no contribution to either $\kappa_{N}$ or $\kappa_{A}$.

Lemma 6.5 (Bounding $\kappa_{N}(\Delta)$ ) Let $G$ be a limit group. Then

$$
\frac{1}{2} \mathrm{~b}_{1}(G) \geq \kappa_{N}(\Delta) \geq 1-\frac{3}{2} \mathrm{~b}_{1}(G)
$$

for all abelian decompositions $\Delta$ of $G$.

Proof Since each valence one vertex group of $\Delta$ contributes at least 1 to $\mathrm{b}_{1}(G)$, we have that $\kappa_{N \mid A} \leq \frac{1}{2} \mathrm{~b}_{1}(G)$ since $\kappa_{N \mid A} \leq \kappa_{N \mid A}^{+}$. By Gauss-Bonnet $\kappa_{N}+\kappa_{A}=$ $1-\mathrm{b}_{1}(\Delta) \geq 1-\mathrm{b}_{1}(G)$ therefore

$$
\kappa_{N}+\frac{1}{2} \mathrm{~b}_{1}(G) \geq 1-\mathrm{b}_{1}(G) .
$$

Thus $\kappa_{N} \geq 1-\frac{3}{2} \mathrm{~b}_{1}(G)$. The same also holds for $\kappa_{A}$.

Define

$$
\operatorname{sc}_{2}(G):=\left(c_{a}(G), c_{g}(G), c_{b}(G), \kappa_{N}(G)\right)
$$


Lemma 6.6 Suppose $\varphi: G \rightarrow H$ is degenerate and indecomposable. Then $\operatorname{sc}_{2}(G) \leq$ $\operatorname{sc}_{2}(H)$. If equality holds then all non-valence-two rigid vertex groups $R$ of $G$ have nonabelian image in $H$, the relative JSJ decompositions $\operatorname{JSJ}(\widehat{\boldsymbol{S}}(R))$ are trees, edge groups incident to $R$ have images in valence one abelian vertex groups of $\operatorname{JSJ}(\hat{\boldsymbol{S}}(R))$, and no two incident edge groups have conjugate centralizer in $\boldsymbol{A S}(R)$. Moreover, in the process of taking the direct $\operatorname{limit} \lim G_{i} \rightarrow H$, at no point do edges incident to rigid vertex groups of $G_{i}$ have conjugate centralizers in the associated rigid vertex groups of $G_{i+1}$ after applying $\boldsymbol{S}_{\mathrm{E}}$.

This argument is used again in the proof of Lemma 8.5.

Proof Suppose $\operatorname{sc}_{1}(G)=\operatorname{sc}_{1}(H)$. Let $R$ be a (nonabelian) rigid vertex. If $R$ has valence one then $R$ doesn't have abelian image. Thus we only need to consider rigid vertices with valence at least three: those with valence two are free to have abelian image without disturbing $\kappa_{N}$. Let $R$ have valence at least three. If $R$ has abelian image in $H$ then $\kappa_{N}$ must increase by at least $\frac{1}{2}$. We only need analyze rigid vertex groups with nonabelian image.

By our standing assumption that JSJ decompositions be bipartite (recall that every edge is adjacent to a maximal abelian vertex group), with one class the abelian vertex groups, the other the nonabelian vertex groups, and if two edge groups are incident to a rigid vertex group $R$, then they have nonconjugate centralizer, we may assume that $\operatorname{JSJ}(\widehat{\boldsymbol{S}}(R))$ has a form such that all images of edge groups incident to $R$ map to abelian vertex groups of $\operatorname{JSJ}(\widehat{\boldsymbol{S}}(R)$ ) (If an incident edge group has image in a rigid vertex group and its centralizer isn't conjugate to the centralizer of an incident edge group in $\operatorname{JSJ}(\widehat{\boldsymbol{S}}(R))$, simply introduce a new edge and pull the centralizer). Moreover, in $\boldsymbol{A S}(G)$, since $c_{a}(G)=c_{a}(H)$, all abelian vertex groups of $\boldsymbol{A S}(G)$ contributed by $\boldsymbol{A} \boldsymbol{S}(R)$ are equal to their peripheral subgroups. In particular, there are no valence one abelian vertex groups which don't contain the image of some edge group incident to $R$. Let $v_{1}, \ldots, v_{n}$ be the vertices of $\operatorname{JSJ}(\widehat{S}(R))$ corresponding to abelian vertex groups, and let $T$ be the subtree of $\operatorname{JSJ}(\widehat{S}(R))$ spanned by the collection $\left\{v_{i}\right\}$, and let $T_{j}$ be the collection of closures of complimentary components of $T$ in $\operatorname{JSJ}(\widehat{\boldsymbol{S}}(R))$. Note that if $T_{j} \cap T_{j^{\prime}} \neq \varnothing$ then the intersection is a single point and coincides with the intersections $T \cap T_{j}$ and $T \cap T_{j^{\prime}}$.

The contribution of $T$ to $\kappa_{N}$ still needs to be computed. First, we claim that if two images of edge groups incident to $R$ have conjugate centralizing elements in $\boldsymbol{A S}(R)$, or if some incident edge group maps to a non valence one vertex of $T$ then

$$
\sum_{v \in T, R_{v} \text { nonabelian }} \kappa(v)>\kappa_{N}(R) .
$$


Equality holds if we pretend that every vertex group from $T$ is nonabelian and that the centralizers of images of incident edge groups are nonconjugate. If any vertex of valence greater than two is abelian, there is inequality. If all vertices of valence at least three are nonabelian, then no two images of incident edge groups can have conjugate centralizers, since these correspond to new abelian vertex groups of $A S(\Delta)$ with valence at least three. If an incident edge group has image in a non-valence one abelian vertex group of $T$ then the corresponding vertex of $\boldsymbol{A S}(\Delta)$ has valence at least three, and the corresponding contribution to the computation of $\kappa_{N}$ is strictly positive.

Now we compute the contribution of the trees $T_{j}$ to $\kappa_{N}$. As above, if we pretend that each tree consists of only nonabelian vertex groups, then the contribution each tree makes to $\kappa_{N}$ over that of $R$ is at least $\frac{1}{2}$. If any vertex groups of valence at least three are abelian, then, since no valence one vertex groups not centralizing images of edge groups incident to $R$ are abelian, the contribution of $T_{j}$ to $\kappa_{N}$ is nonzero.

To verify that $\kappa_{N}(G) \leq \kappa_{N}(H)$ we only need to check that iterated application of $\boldsymbol{S}_{\mathrm{A}} \circ \boldsymbol{S}_{\mathrm{E}}$ to $\boldsymbol{A S}(G)$ cannot decrease $\kappa_{N}$. This follows immediately from the fact that no rigid vertex group of $\boldsymbol{A S}(G)$ splits after an application of $\boldsymbol{S}_{\mathrm{E}}$ (Lemma 5.21) and the observation that folding edges of nonabelian vertex groups with conjugate centralizers can only increase $\kappa_{N}$. If $\kappa_{N}(H)=\kappa_{N}(G)$ then no such folding occurs.

When crushing trees (the total genus cannot increase since $c_{q}(G)=c_{q}(H)$ ) of QH and subsurface groups from the abelian decomposition $H$ inherits from $A S(G)$ to build the QH subgroups of $H$, as in the proof of Lemma $6.2, \kappa_{N}$ doesn't change.

The final step in this coarse analysis of the degenerations of JSJ decompositions is the following observation. Define the total complexity of QH subgroups:

$$
c_{q}(G):=\sum_{Q \mathrm{QH}}|\chi(Q)|
$$

Lemma 6.7 If $\operatorname{sc}_{2}(G)=\operatorname{sc}_{2}(H)$ then

$$
c_{q}(G) \leq c_{q}(H) .
$$

If equality holds then no vertex groups of $\boldsymbol{A S}(R)$ correspond to subsurface groups of $\mathrm{QH}$ vertex groups of $H$.

Proof Since $\kappa_{N}(G)=\kappa_{N}(H)$, no nonabelian vertex group with valence one or at least three has abelian image. If $\boldsymbol{A S}(R)$ contributes a subsurface group of a $\mathrm{QH}$ subgroup of $H$ then the total Euler characteristic must decrease since the subsurface group is either a multiply punctured sphere or projective plane with Euler characteristic at most -1 . 
Definition 6.8 (Essential, vulnerable) We say that a vertex of the abelian JSJ decomposition of a limit group is essential if it satisfies at least one of the following:

- It is QH.

- It isn't valence two.

- It is abelian and isn't equal to its peripheral subgroup.

Denote by $v_{e}(G)$ the number of essential vertices of $G$, and let $\beta(G)$ be the set of essential vertices in $\operatorname{JSJ}(G)$, and let $p(G)$ be the set of unoriented reduced edge paths $\alpha:[0,1] \rightarrow \operatorname{JSJ}(G)$ such that $\alpha^{-1}(\beta(G))=\{0,1\}$. The elements of $p(G)$ fall into six classes, corresponding to the types of endpoints: for example, $A A(G)$ is the set of elements of $p(G)$ whose endpoints are both essential abelian vertices. A path in $A A(G), A Q(G)$, or $Q Q(G)$, is vulnerable if it crosses a rigid vertex group. Let $c_{v}(G)$ be the number of vulnerable paths in $A Q(G) \sqcup Q Q(G)$, and let $c_{\# r}(G)$ be the number of essential rigid vertex groups of $G$.

Definition 6.9 (Complexity) The complexity of a freely indecomposable limit group is the lexicographically ordered tuple

$$
\operatorname{sc}(G):=\left(c_{a}(G), c_{g}(G), c_{b}(G), \kappa_{N}(G), c_{q}(G), c_{\# r}(G),-v_{e}(G),-c_{v}(G)\right) .
$$

The number of values this complexity takes is controlled by the first betti number. That the last four take only boundedly many values follows from Lemma 2.27, bounding the complexity of the JSJ decomposition in terms of $\mathrm{b}_{1}$ (controlling both $c_{q}$ and $c_{\# r}$ ), and Lemma $6.5\left(\kappa_{N}\right)$.

Fix a degenerate and indecomposable map $\varphi: G \rightarrow H$ such that $\operatorname{sc}_{2}(G)=\operatorname{sc}_{2}(H)$. Then $G$ and $H$ satisfy the conclusions of Lemma 6.6. If, additionally, $c_{q}(G)=c_{q}(H)$, then by Lemma 6.7, for all rigid vertex groups $R$ of $G, \boldsymbol{A S}(R)=\widehat{\boldsymbol{S}}(R) *_{F_{j}} B_{j}$, no vertex group of $\operatorname{JSJ}(\widehat{\boldsymbol{S}}(R))$ is a subsurface group of a QH subgroup of $H$, and we conclude by Lemma 5.21 that all rigid vertex groups of $H$ are obtained by iteratively adding roots to rigid vertex groups from the collection $\left\{A S\left(R_{i}\right) \mid R_{i}\right.$ rigid in $\left.G\right\}$. If the relative JSJ of some $\boldsymbol{A S}(R)$ has more than one vertex of valence three, $c_{\# r}(G)<$ $c_{\# r}(H)$. Thus, if $\operatorname{sc}(G)=\operatorname{sc}(H)$ then, for all rigid vertex groups $R_{i}$, the underlying graph of $\operatorname{JSJ}\left(\widehat{\boldsymbol{S}}\left(R_{i}\right)\right)$ is a tree, has only one vertex of valence at least three, the valence of that vertex is precisely the valence of $R$, and every valence one vertex contains the image of exactly one incident edge group from $G$.

Let

$$
\operatorname{sc}_{2}(G)=\left(c_{a}(G), c_{g}(G), c_{b}(G), \kappa_{N}(G), c_{q}(G), c_{\# r}(G)\right)
$$

and suppose that $\operatorname{sc}_{2}(G)=\operatorname{sc}_{2}(H)$ and $-v_{e}(G)<-v_{e}(H)$. There must be a path $p$ in $A A(G)$ or $Q Q(G)$ such that every rigid vertex group crossed has abelian image 
in $H$. (No path in $A Q(G)$ contains an essential vertex, so we don't have to consider this case.) Abelianizing these groups leaves $\kappa_{N}$ and $c_{q}$ unchanged. If $p \in A A(G)$ then the number of essential abelian vertices of $H$ is less than that of $G$. Now we consider the quantity $c_{v}$, under the assumption that $-v_{e}$ is constant. The number of vulnerable paths cannot increase, as the topology and labeling of essential vertices of the underlying graphs of JSJ's is unchanged under $G \rightarrow H$. The next lemma follows immediately from the definitions.

Lemma 6.10 If $\operatorname{sc}(G)=\operatorname{sc}(H)$ then at least one rigid vertex group of $G$ crossed by a vulnerable path in $A Q(G) \sqcup Q Q(G) \sqcup A A(G)$ has nonabelian image in $H$.

Definition 6.11 (Weakly JSJ-respecting) If $\varphi: G \rightarrow H$ is degenerate and $\operatorname{sc}(G)=$ $\operatorname{sc}(H)$, then $\varphi$ is weakly $\mathrm{JSJ}-$ respecting.

In conclusion, we have the following. Let $C_{n}$ be the number of values sc takes for limit groups with first betti number $n$.

Theorem 6.12 Let $\mathcal{L}$ be an indecomposable degenerate chain of limit groups. If $\|\mathcal{L}\|>K \cdot C_{\mathrm{b}_{1}(\mathcal{L})}$ then there is a weakly JSJ-respecting subchain of $\mathcal{L}$ of length $K . \square$

We can now define the GAD's which are aligned under maps that don't increase the complexity.

Definition 6.13 ( $\Delta$-admissible, $\Delta$-stable, almost JSJ-respecting) A virtual JSJ decomposition of a limit group $L$ is an abelian decomposition $\Delta$ such that the complexity of $L$ measured with respect to $\Delta$ is the same as the complexity of $L$ measured with respect to $\operatorname{JSJ}(L)$.

Let $\varphi: G \rightarrow H$. If $\Delta$ is a virtual JSJ decomposition of $G$ then $\varphi$ is $\Delta$-admissible if it is degenerate, $\operatorname{sc}(G)=\operatorname{sc}(H)$, and every nonabelian rigid vertex group of $\Delta$ has nonabelian image in $H$. Let $\mathcal{L}$ be a weakly JSJ-respecting chain, and let $\Delta$ be a virtual JSJ decomposition of $\mathcal{L}(1)$. We say that $\mathcal{L}$ is $\Delta$-admissible if all $\varphi_{1, j}$ are $\Delta$-admissible. Suppose $\varphi: G \rightarrow H$ is $\Delta$-admissible. Then $\varphi$ is $\Delta$-stable if, for every rigid vertex group $R$ of $\Delta, c_{a}\left(\left.\varphi\right|_{R}, \mathcal{E}(R)\right)=0$ (see Definition 5.11).

Let $\mathcal{L}$ be a weakly JSJ-respecting chain such that $\varphi_{i, j}$ is $\operatorname{JSJ}(\mathcal{L}(i))$-stable for all $i$ and $j$. If $\mathcal{L}(i)$ and $\mathcal{L}(j)$ have the same number of rigid vertex groups for all $i$ and $j$, and the betti numbers of rigid vertex groups of JSJ decompositions are constant, then $\mathcal{L}$ is almost JSJ-respecting. 
If $\varphi$ is $\Delta$-admissible then there is a pushforward of $\Delta$ to $H$ that is also a virtual JSJ decomposition. The pushforward of $\Delta$ is obtained from the JSJ of $H$ by collapsing the forest spanned by the nonabelian vertex groups coming from $\operatorname{JSJ}(\widehat{\boldsymbol{S}}(R))$, as $R$ varies over all rigid vertex groups of $\Delta$. The pushforward is not necessarily the same as $\boldsymbol{S}(\Delta)$. It should be noted that since $\varphi$ is degenerate it doesn't matter if we use $\Delta$ or the JSJ of $G$ when constructing $\boldsymbol{A S}(G)$.

Let $\Delta$ be a virtual JSJ decomposition of $G$. We construct a virtual JSJ $\varphi_{*}(\Delta)$ for $H$. Rather than constructing $H$ from $G$, first by applying strict vertex morphisms to rigid vertex groups from the JSJ of $G$, we apply them only to the vertex groups of the virtual decomposition $\Delta$. Let $R$ be a rigid vertex group of $\Delta$, and construct $\boldsymbol{A S}(R, \mathcal{E}(R)) \cong \widehat{\boldsymbol{S}}(R) * F_{j} B_{j}$ as in the construction of $\boldsymbol{A S}(G)$ with respect to $\Delta$. Note that if $c_{a}\left(\left.\varphi\right|_{R}, \mathcal{E}\right)>0$, ie, if $\varphi$ is not $\Delta$-stable, $\mathrm{b}_{1}(\widehat{\boldsymbol{S}}(R))<\mathrm{b}_{1}(R)$.

Now consider the relative JSJ decomposition of $\widehat{\boldsymbol{S}}(R)$. Since $\Delta$ is a virtual JSJ decomposition of $G$, the relative JSJ of $\hat{\boldsymbol{S}}(R)$ has at most one essential vertex and that vertex has the same valence as $R$. If $\operatorname{JSJ}\left(\widehat{\boldsymbol{S}}\left(R,\left\{F_{j}\right\}\right)\right)$ contains more than one essential vertex then $\operatorname{sc}(G)<\operatorname{sc}(H))$.

Consider the direct $\operatorname{limit} \lim G_{n}=H$. By Lemma 5.21 the rigid vertex groups of $H$ are obtained by iteratively adjoining roots to the vertex groups of $\widehat{\boldsymbol{S}}(R)$, as $R$ varies over all vertex groups of $\Delta$. Since the complexity doesn't increase, nonconjugacy of incident edge groups is maintained and no inessential vertex group of $G$ can give rise to a essential vertex of $\operatorname{JSJ}(H)$. For each rigid vertex group of $\Delta$, declare the subtree of groups of the JSJ decomposition of JSJ $(H)$ coming from $\widehat{\boldsymbol{S}}(R)$ a vertex group of an abelian decomposition $\varphi_{*}(\Delta)$ of $H$. The decomposition $\varphi_{*}(\Delta)$ is obviously a virtual JSJ decomposition. Call the vertex of $\varphi_{*}(\Delta)$ associated to $R$, the push forward of $R, \varphi_{*}(R)$.

If $c_{a}\left(\left.\varphi\right|_{R}, \mathcal{E}(R)\right)>0$, then $\mathrm{b}_{1}(\boldsymbol{S}(R))<\mathrm{b}_{1}(R)$. Since $\varphi_{*}(R)$ is obtained by iteratively adjoining roots to $\boldsymbol{S}(R)$, the betti number cannot increase and $\mathrm{b}_{1}\left(\varphi_{*}(R)\right)<\mathrm{b}_{1}(R)$.

The pushforward satisfies a kind of functoriality. If $\varphi: G \rightarrow H$ is $\Delta$-admissible, and if $\psi: H \rightarrow K$ is degenerate, and $\psi \varphi$ is degenerate, then $\psi$ is $\varphi_{*}(\Delta)$-admissible and $(\psi \varphi)_{*}(\Delta)=\psi_{*}\left(\varphi_{*}(\Delta)\right)$, likewise for pushforwards of vertex groups.

Lemma 6.14 A virtual JSJ decomposition is obtained by collapsing a forest $F$ in $\operatorname{JSJ}(G)$ of the following form, and all such forest collapses yield virtual JSJ decompositions:

- All valence one vertices of $F$ are rigid, all valence two vertices are rigid or abelian. 
- Each component of $F$ contains at most one essential vertex. If $F$ contains an essential vertex then that vertex is rigid. Every component of $F$ is star-shaped, and all non-valence-two vertices correspond to rigid vertex groups of the JSJ of $G$.

If $G \rightarrow H$ is degenerate, indecomposable, and $\operatorname{sc}(G)=\operatorname{sc}(H)$ then the JSJ decompositions of $G$ and $H$ resemble one another quite strongly. The next step is to show that in weakly JSJ-respecting chains, one can choose, uniformly in $b_{1}$, long subchains such that rigid vertex groups have nonabelian images and are obtained from images of earlier rigid vertex groups by iteratively adjoining roots and passing to limit group quotients.

Lemma 6.15 There exists $M=M(K, n)$ such that if $\mathcal{L}$ is $\Delta$-admissible degenerate chain with $\|\mathcal{L}\|_{\mathrm{pl}} \geq M\left(K, \mathrm{~b}_{1}(\mathcal{L})\right)$, then there is a subchain $\mathcal{L}^{\prime}$ of $\mathcal{L}$ such that $\left\|\mathcal{L}^{\prime}\right\|_{\mathrm{pl}} \geq K$ and $\mathcal{L}^{\prime}$ is either almost JSJ-respecting or there is a decomposition $\Delta^{\prime}$ of $\mathcal{L}^{\prime}(1)$ with strictly more rigid vertex groups than $\Delta$ and $\mathcal{L}^{\prime}$ is $\Delta^{\prime}$-stable.

Proof We start by defining virtual JSJ decompositions $\Delta_{i}=\left(\varphi_{1, i}\right)_{*}(\Delta)$. Let $b_{n}$ be the largest betti number of a vertex group in any virtual JSJ decomposition of a limit group with first betti number $n$. Let $M=2 \cdot K \cdot b_{n} \cdot \operatorname{Verts}(n)$. If $\mathrm{b}_{1}(\mathcal{L})=n$ and $\|\mathcal{L}\|>M$ then $\mathcal{L}$ has a subchain $\mathcal{L}^{\prime}$ of length $K$ such that for each rigid $R_{i, j}$ $c_{a}\left(\left.\varphi_{i, i+1}\right|_{R_{i, j}}, \mathcal{E}\left(R_{i, j}\right)\right)=0$. In particular $\mathcal{L}^{\prime}$ is $\Delta^{\prime}$-stable, where $\Delta^{\prime}$ is the pushforward of $\Delta$ to $\mathcal{L}^{\prime}(1)$.

Let $k$ be the last index for which the push forward of $\Delta^{\prime}$ to $\mathcal{L}^{\prime}(k)$ is $\mathcal{L}^{\prime}(k)$ 's abelian JSJ decomposition, and let $\mathcal{L}_{H}^{\prime}$ and $\mathcal{L}_{T}^{\prime}$ be the two subchains of $\mathcal{L}^{\prime}$ obtained by restricting to the first $k$ and last $\left\|\mathcal{L}^{\prime}\right\|-k$ indices, respectively ( $k$ may be 0 or $\left\|\mathcal{L}^{\prime}\right\|$ ).

There is a virtual JSJ decomposition $\Delta^{\prime}$ of $\mathcal{L}(k+1)$ for which $\mathcal{L}_{T}^{\prime}$ is $\Delta^{\prime}$-admissible and the number of vertex groups of $\Delta^{\prime}$ is strictly greater than the number of vertex groups of $\Delta$ : examine $\mathcal{L}_{T}^{\prime}$. There must be a vertex group $R$ of $\mathcal{L}_{T}^{\prime}(1)$ such that $\operatorname{JSJ}(R, \mathcal{E}(R))$ is nontrivial, that is, $R$ must have a splitting $R=R^{\prime} *_{E} A *_{E^{\prime}} R^{\prime \prime}, R^{\prime}$ and $R^{\prime \prime}$ nonabelian, $A=\left\langle E, E^{\prime}\right\rangle$ maximal abelian (recall that $A$ is the centralizer of its incident edge groups), and with all edge groups incident to $R$ elliptic and not centralized by $A$. Then neither $R^{\prime}$ nor $R^{\prime \prime}$ has abelian image in any $\mathcal{L}_{T}^{\prime}(l)$ : if this happened then, since limit groups have nontrivial homology relative to any abelian subgroup, the map $R \rightarrow \mathcal{L}_{T}^{\prime}(l)$ would factor through (say) $R^{\prime} *_{E} A *_{E^{\prime}}\left(R^{\prime \prime}\right)^{\text {ab }}$, which has nonzero $c_{a}$ since the edges incident to $R$ are elliptic. Since $\varphi_{1, k+1 *}\left(\Delta^{\prime}\right)$ is obtained by collapsing a forest, removing the edges labeled $E$ and $E^{\prime \prime}$ from the forest gives a new forest which yields a new virtual JSJ decomposition $\Delta^{\prime \prime}$ of $\mathcal{L}_{T}^{\prime}(1)$. The new decomposition 
has more vertices than $\Delta$, and, since neither $R^{\prime} \rightarrow \mathcal{L}_{T}^{\prime}(l)$ nor $R^{\prime \prime} \rightarrow \mathcal{L}_{T}^{\prime}(l)$ has abelian image for any $k$, the chain $\mathcal{L}_{T}^{\prime}$ is $\Delta^{\prime \prime}$-admissible. At least one of $\mathcal{L}_{T}^{\prime}$ or $\mathcal{L}_{H}^{\prime}$ has length at least $K$.

We use Lemma 6.15 to control those degenerations of JSJ decompositions which are invisible to sc.

Theorem 6.16 There is $M=M(K, n)$ such that if $\mathcal{L}$ is weakly JSJ-respecting and $\|\mathcal{L}\|>M\left(K, \mathrm{~b}_{1}(\mathcal{L})\right)$, then there is an almost-JSJ-respecting subchain $\mathcal{L}^{\prime}$ of $\mathcal{L}$ of length at least $K$.

Proof We need to show that such chains are $\Delta$-admissible for some $\Delta$. Lemma 6.10 implies that no rigid vertex groups crossed by vulnerable paths in $A Q(\mathcal{L}(1))$ or $Q Q(\mathcal{L}(1))$ might have abelian image in some $\mathcal{L}(k)$. We construct a forest in $\operatorname{JSJ}(\mathcal{L}(1))$ as follows. Let $p$ be a vulnerable path, and let $p^{\prime}$ be a longest proper subpath of $p$ with endpoints which are rigid vertex groups of $G$. The subgraph of groups spanned by the image of $p^{\prime}$ doesn't have abelian image in any $\mathcal{L}(k)$, by Lemma 6.10.

For $p \in R R(\mathcal{L}(1)) \sqcup R A(\mathcal{L}(1)) \sqcup R Q(\mathcal{L}(1))$ choose an orientation of $p$ let $p^{\prime}$ be the subpath which begins at $p(1)$ and ends at the last inessential rigid vertex group crossed by $p$.

The union of all images of $p^{\prime}$ 's is a forest and defines a virtual JSJ decomposition $\Delta$ of $\mathcal{L}(1)$ by Lemma 6.14. The chain $\mathcal{L}$ is $\Delta$-admissible.

Let $f(K)=M\left(K, \mathrm{~b}_{1}(\mathcal{L})\right)$ be the bound from Lemma 6.15. If $\|\mathcal{L}\|>f^{\operatorname{Verts}(n)}(K)$ then $\mathcal{L}$ is long enough to apply the lemma $\operatorname{Verts}(n)$ times, yielding an almost JSJ-respecting subchain of length $K$.

We introduce some technical refinements of the notion of almost JSJ-respecting which are used in Section 8.

Definition 6.17 Recall that the neighborhood of $V, \mathcal{N}(V)$, is the subgroup generated by $V$ and conjugates of non-QH adjacent vertex groups which intersect $V$ nontrivially (Definition 2.4). A GAD $\Delta$ of a limit group $L$ misses $A$ if $A$ is an abelian vertex group of $\operatorname{JSJ}(L)$ and the neighborhood of $A$ is elliptic in $\Delta$. Any GAD of $L$ missing $A$ is clearly obtained from $\operatorname{JSJ}(L, \mathcal{N}(A))$ by cutting $\mathrm{QH}$ vertex groups along a family of disjoint simple closed curves, folding, and collapsing subgraphs.

If $\mathcal{A}$ is a collection of abelian vertex groups of $L$ then the GAD of $L$ missing all elements $\mathcal{A}$ is denoted by $\operatorname{JSJ}(L ;\{\mathcal{N}(A) \mid A \in \mathcal{A}\})$. It follows from the definition of $\boldsymbol{S}$ that if $\varphi: G \rightarrow H$ is degenerate and almost-JSJ respecting then the vertex groups of $\mathrm{JSJ}\left(H,\left\{\mathcal{N}(A) \mid A \in \phi_{\#}(\mathcal{A})\right\}\right)$ are obtained from the images of the vertex groups of $\mathrm{JSJ}(G ;\{\mathcal{N}(A) \mid A \in \mathcal{A}\})$ by iteratively adjoining roots. 
For example, the principal cyclic JSJ is the decomposition missing $\{A \mid P(A)$ not cyclic $\}$.

Definition 6.18 (JSJ-respecting) A chain $\mathcal{L}$ is JSJ-respecting if it is almost JSJ-respecting chain satisfying $\mathrm{b}_{1}(R)=\mathrm{b}_{1}\left(\varphi_{i, j, \#}(R)\right)$ for all nonabelian non-QH vertex groups $R$ of $\operatorname{JSJ}(\mathcal{L}(i),\{\mathcal{N}(A) \mid A \in \mathcal{A}\})$, as $\mathcal{A}$ varies over all collections of abelian vertex groups of $\mathcal{L}(i)$.

The the next theorem follows immediately from Theorems 6.12 and 6.16.

Theorem 6.19 There is $M=M(K, n)$, such that if $\mathcal{L}$ is almost JSJ-respecting and $\|\mathcal{L}\| \geq M\left(K, \mathrm{~b}_{1}(\mathcal{L})\right)$, then there is a JSJ-respecting subchain $\mathcal{L}^{\prime}$ of $\mathcal{L}$ of length at least $K$.

\section{QPJSJ-respecting}

First we bound ranks of abelian subgroups. As a consequence, we may assume that subsequences of abelian subgroups of degenerate JSJ-respecting chains are well behaved.

Lemma 7.1 Let $G$ be a nonabelian limit group with $\mathrm{b}_{1}(G)=n$. Then all abelian subgroups of $G$ have rank at most $n-1$. If $G \rightarrow H, A<G$ abelian, if $\mathbb{Z}^{2}<$ $\operatorname{ker}(A \rightarrow H)$ then $\mathrm{b}_{1}(G)>\mathrm{b}_{1}(H)$.

Proof Let $G=L_{0} \rightarrow L_{1} \rightarrow \cdots$ be a strict resolution of $G$, and let $A<G$ be an abelian subgroup. If $A$ is cyclic then the lemma holds. Suppose $A$ has rank at least two. Since $A$ isn't infinite cyclic it is elliptic in the abelian JSJ decomposition of $G$, and is contained in an abelian vertex group or a rigid vertex group. If the image of $A$ is contained in a rigid vertex group at each stage of the resolution $L_{0} \rightarrow \cdots$ then, since the last group in the strict resolution is free, $A$ must be cyclic. Let $i_{0}$ be the first index such that $A$ is contained in an abelian vertex group $B$ but not completely contained in $P(B)$. If $L_{i_{0}}$ is freely decomposable then we are done by induction since $A$ is contained in a free factor of $L_{i_{0}}$. By linear algebra

$$
\operatorname{rk}(A \cap P(B))+\operatorname{rk}(B / P(B)) \geq \operatorname{rk}(A) .
$$

Let $C$ be a complementary direct summand such that $A \cong(A \cap P(B)) \oplus C$. The summand $C$ has rank at most $\operatorname{rk}(B / P(B))$ and every element of $B / P(B)$ represents a nontrivial element of $\mathrm{H}^{1}\left(L_{i_{0}} ; L_{i_{0}, P}\right)$. Continue this process on $L_{i_{0}, P}$ with respect to $A \cap P(B)$, removing direct summands until $A \cap P(B)$ has rank 1. Since $P(B) \neq B$, $\mathrm{b}_{1}\left(L_{i_{0}, P}\right)<\mathrm{b}_{1}\left(L_{i_{0}}\right)$ and $\operatorname{rk}(A \cap P(B)) \leq n-\operatorname{rk}(B / P(B))$. The statement clearly holds when $A \cap P(B)$ has rank 1 . 
Suppose $\mathcal{L}$ is JSJ-respecting. Let $\mathcal{A}_{i}$ be the sequence

$$
\left(A_{i}^{n} \rightarrow A_{i}^{n+1}\right),
$$

where $A_{i}^{n}$ is the $i$-th abelian vertex group of $\mathcal{L}(n)$, ordered so that $\varphi_{n, n+1}\left(A_{i}^{n}\right)<A_{i}^{n+1}$. Abbreviate $A_{i}^{n}$ by $\mathcal{A}_{i}(n)$.

The homomorphism $\varphi_{n, m}$ may not be injective on $A_{i}^{n}$, but since $\varphi_{n, m}$ doesn't factor through a free product, the image is not trivial.

Definition 7.2 A degenerate JSJ-respecting chain $\mathcal{L}$ is QPJSJ-respecting if, for each sequence $\mathcal{A}_{i}$, and for sequences of edge spaces as well, one of the following holds:

- $\quad P\left(\mathcal{A}_{i}(n)\right)$ is infinite cyclic for all $i$ and $n$.

- $\quad P\left(\mathcal{A}_{i}(n)\right)$ has rank 2 and $\operatorname{ker}\left(P\left(\mathcal{A}_{i}(n)\right) \rightarrow P\left(\mathcal{A}_{i}(n+1)\right)\right)$ is infinite cyclic for all $n$.

- $\quad P\left(\mathcal{A}_{i}(n)\right)$ doesn't satisfy either of the above conditions for any $n$.

We require that if $\mathcal{L} \square_{\rho} \mathcal{G}$ is a QPJSJ-respecting maximal resolution then $\operatorname{depth}(\mathcal{L})=$ $\operatorname{depth}(\rho(\mathcal{L}(i)))$ for all $i$.

A sequence of edge or peripheral subgroups is big if it satisfies the third bullet, and small otherwise. An edge or peripheral subgroup is big or small if it is contained in a big or small sequence, respectively. Sequences satisfying the second bullet are flexible.

Lemma 7.3 There is $M=M(K, n)$ such that if $\mathcal{L}$ is a degenerate JSJ-respecting sequence and $\|\mathcal{L}\|>M\left(K, \mathrm{~b}_{1}(\mathcal{L})\right)$, then $\mathcal{L}$ has a QPJSJ-respecting subsequence of length at least $K$.

Proof Immediate. The ranks obey $\operatorname{rk}\left(\mathcal{A}_{i}(n)\right) \geq \operatorname{rk}\left(\mathcal{A}_{i}(m)\right)-1$ for all $i$ and $n$, the ranks are bounded above by $b_{1}(\mathcal{L})$ (Lemma 7.1), and by Lemma 2.27 the number of edge groups is controlled by $b_{1}(\mathcal{L})$.

Definition 7.4 Degenerate QPJSJ-respecting chains are designed so that certain important sequences of subgroups are respected under all maps. Let $\mathcal{L}$ be QPJSJ-respecting. Let $\mathcal{B}=\left\{\mathcal{B}_{i}\right\}$ be the collection of sequences of abelian vertex groups such the peripheral subgroups $P\left(\mathcal{B}_{i}(n)\right)$ are big for $\mathcal{B}_{i} \in \mathcal{B}$. Define

$$
\operatorname{QPJSJ}(\mathcal{L}(n)):=\operatorname{JSJ}\left(\mathcal{L}(n),\left\{\mathcal{N}\left(\mathcal{B}_{i}(n)\right) \mid \mathcal{B}_{i} \in \mathcal{B}\right\}\right) .
$$

$\operatorname{QPJSJ}(\mathcal{L}(n))$ is the quasiprincipal JSJ decomposition of $\mathcal{L}(n)$, or QPJSJ, for short. 
Let $\left\{R_{i}^{k}\right\}$ be the collection of vertex groups in $\operatorname{QPJSJ}(\mathcal{L}(k))$. The map $\varphi_{k, l}$ sets up a one to one correspondence between abelian vertex groups with big|small peripheral subgroups of $\operatorname{JSJ}(\mathcal{L}(k))$ and $\operatorname{JSJ}(\mathcal{L}(l))$, and the induced maps $\mathcal{L}(k) \rightarrow \mathcal{L}(l)$ respect vertex groups and adjacency of edges, thus there is a one-to-one correspondence between vertex groups of $\operatorname{QPJSJ}(\mathcal{L}(k))$ and $\operatorname{QPJSJ}(\mathcal{L}(l))$, ie, there is a unique vertex group $R_{i}^{l}$ in $\operatorname{QPJSJ}(\mathcal{L}(l))$ such that $\varphi_{k, l}\left(R_{i}^{k}\right)<R_{i}^{l}$.

Define the following sequences of images of vertex groups of $\mathcal{L}$ :

$$
\mathcal{R}_{i}^{n}(m):=\varphi_{n, m}\left(R_{i}^{n}\right)
$$

There are obvious inclusions $\mathcal{R}_{i}^{n} \hookrightarrow \mathcal{L}$.

Lemma 7.5 Let $\mathcal{R}_{i}^{(n)}$ be as above.

- $\mathcal{R}_{i}(n+1)=\mathcal{R}_{i}^{n+1}(n+1)$ is obtained by iteratively adjoining roots to $\mathcal{R}_{i}^{n}(n+1)$.

- $\mathrm{b}_{1}\left(R_{i}^{n}\right) \leq \mathrm{b}_{1}(\mathcal{L}(n))$.

- If $\mathrm{b}_{1}\left(\mathcal{R}_{i}\right)=\mathrm{b}_{1}(\mathcal{L})$ for some $i$ then there is only one nonabelian vertex in each QPJSJ decomposition, and $A=P(A)$ for every abelian vertex group in a small sequence of abelian vertex groups.

Proof The first bullet follows from the fact that the vertex groups of $\operatorname{QPJSJ}(\mathcal{L}(n))$ are generated by vertex groups and stable letters from $\operatorname{JSJ}(\mathcal{L}(n))$, that the vertex groups of $\operatorname{JSJ}(\mathcal{L}(n+1))$ are obtained from the images of vertex groups of $\operatorname{JSJ}(\mathcal{L}(n))$ by iteratively adjoining roots, by Lemma 5.21 . The construction is the same as the pushforward defined for JSJ-stable maps.

The inequality of betti numbers follows from the fact that the map on the peripheral subgroups of $\operatorname{QPJSJ}(\mathcal{L}(N))$ to first homology have one-dimensional image, and the third bullet follows from the same argument.

The following follow from Theorem 6.19 and Lemma 7.3.

Theorem 7.6 (Alignment theorem) There is $M=M(K, n)$ such that if $\mathcal{L}$ is an indecomposable sequence of limit groups, $\|\mathcal{L}\|>M(K, \operatorname{rk}(\mathcal{L}))$, then there is a maximal surjective indecomposable QPJSJ-respecting resolution $\widetilde{\mathcal{L}} \square \mathcal{L}$ with $\|\widetilde{\mathcal{L}}\| \geq K$.

By Lemma 2.28 we have the following corollary.

Corollary 7.7 (Alignment corollary) There is $M=M(K$, Comp) such that if $(\iota: \mathcal{G} \hookrightarrow \mathcal{L})$ is indecomposable, $\|\mathcal{G}\|>M(K, \operatorname{Comp}(\iota))$, then there is a maximal surjective QPJSJ-respecting resolution $\widetilde{\mathcal{G}} \square \mathcal{G}$ with $\|\widetilde{\mathcal{G}}\| \geq K$. 


\section{Decreasing the complexity}

Recall that the principal cyclic analysis lattice of a limit group is obtained by iteratively passing either to freely indecomposable free factors or to vertex groups of the principal cyclic JSJ. Alignment of QPJSJ decompositions and Grushko decompositions in maximal resolutions lets us mimic this construction for sequences of limit groups.

Definition 8.1 The nonprincipal JSJ of a freely indecomposable limit group $L$, $\operatorname{NPJSJ}(L)$, without $\mathrm{QH}$ vertex groups is the abelian decomposition of $L$ obtained by collapsing all edges of the abelian JSJ decomposition of $L$ connected to abelian vertex groups $A$ with $P(A) \cong \mathbb{Z}$.

The nonprincipal JSJ of a freely indecomposable limit group $\mathcal{P}(n)$ in a $\mathrm{QH}-$ free QPJSJ-respecting sequence $\mathcal{P}$ is obtained by collapsing all edges of the abelian JSJ decomposition of $\mathcal{P}(n)$ connected to abelian vertex groups $A$ such that $P(A)$ has cyclic image (the small sequences of vertex groups) in $\mathcal{P}(n+1)$. In other words, the JSJ decomposition associated to the set of splittings in which all neighborhoods of small abelian vertex groups are elliptic.

Lemma 8.2 Let $\varphi: P \rightarrow L$ be an indecomposable map of limit groups; $c_{a}(L)=0$. Let $\Delta=\Delta\left(V_{i}, A_{j}, E_{k}\right)$ be a decomposition of $P$ over abelian edge groups $E$, nonabelian vertex groups $V$, and abelian vertex groups $A$, such that every edge $E$ connects a vertex $V$ to a vertex $A$. Suppose that:

- Every splitting of $P$ over a subgroup of some $A_{j}$ is nonprincipal.

- $\varphi\left(P\left(A_{j}\right)\right)$ is noncyclic for each abelian vertex group $A_{j}$.

- $\varphi(P)$ is not elliptic in $\operatorname{PJSJ}(L)$.

Then for some nonabelian vertex group $V, \varphi(V)$ intersects two distinct conjugates of (not necessarily distinct) vertex groups of $\operatorname{PJSJ}(L)$ in nonabelian subgroups, and edge groups incident to $V$ have elliptic image in $L$.

Proof Suppose that each vertex group $V$ has elliptic image in $\operatorname{PJSJ}(L)$. The map $\varphi$ sends each vertex group $A$ to an abelian subgroup of $L$ over which there are no principal cyclic splittings. If $\varphi(P)$ is not elliptic in $\operatorname{PJSJ}(L)$ then some vertex group $V$ has to act hyperbolically in the associated tree, but then $\varphi(V)$ splits relative to the image of $\mathcal{E}(V)$. Since $c_{a}(L)=0$ all vertex groups of this splitting are either nonabelian or cyclic, and any cyclic vertex group must be attached to at least two nonabelian vertex groups. 
Lemma 8.3 Let $\mathcal{G} \square \mathcal{L}$ be a maximal surjective QPJSJ-respecting resolution, with $c_{a}(\mathcal{L}(n))=c_{a}(\mathcal{G}(n))=0$. Let $\mathcal{R}$ be a sequence of images of vertex groups of $\operatorname{QPJSJ}(\mathcal{G})$, and suppose that $\mathcal{P}$ is an indecomposable maximal QPJSJ-respecting resolution of the image $\operatorname{Im}(\mathcal{R}) \hookrightarrow \mathcal{L}$ and $c_{a}(\mathcal{P}(n))=0$. Suppose further that $\mathcal{P}$ has no QH sequences of vertex groups. Then for $n \geq 2$ the vertex groups of $\operatorname{NPJSJ}(\mathcal{P}(n))$ have images contained in vertex groups of $\operatorname{JSJ}(\mathcal{G}(n+1))$ under the natural map $\mathcal{P}(n) \rightarrow \mathcal{G}(n+1)$.

Proof The map $\mathcal{P}(n-2) \rightarrow \mathcal{P}(n)$ is indecomposable, degenerate, and factors through $\mathcal{R}(n-1)$, hence the map $\mathcal{R}(n-1) \rightarrow \mathcal{P}(n)$ is degenerate. Let $\Delta=\Delta\left(V_{i}, A_{j}, E_{k}\right)$ be the decomposition of $\mathcal{R}(n-1)$ induced by $\mathcal{G}(n-1)$, and consider the almost-strict map $\boldsymbol{A S}(\mathcal{R}(n-1)) \rightarrow \mathcal{P}(n)$, built with respect to $\Delta$. Since $\mathcal{R}(n-1) \rightarrow \mathcal{P}(n)$ is degenerate and the images of the $A_{j}$ are nonconjugate $\mathcal{G}(n+1)$, hence nonconjugate in $\mathcal{P}(n)$, we may mimic the construction of the pushforward of an abelian decomposition for $\Delta$-admissible maps. The pushforward of $\Delta$ is an abelian decomposition $\Delta^{\prime}=$ $\Delta^{\prime}\left(V_{i}^{\prime}, A_{j}^{\prime}, E_{k}^{\prime}\right)$ of $\mathcal{P}(n)$, modeled on $\Delta$ with each nonabelian vertex group $V_{i}^{\prime}$ of $\Delta^{\prime}$ obtained from $\widehat{S}\left(V_{i}\right)$ by iteratively adjoining roots. Since $c_{a}(\mathcal{P})=0$, and $P\left(A_{j}^{\prime}\right)$ is noncyclic (recall that $P\left(A_{j}\right)$ is big), there are no splittings from $\operatorname{QPJSJ}(\mathcal{P}(n))$ over subgroups of any $A_{j}^{\prime}$, and each nonabelian vertex group of $\operatorname{NPJSJ}(\mathcal{P}(n))$ is therefore contained in some $V_{i}^{\prime}$. Since $V_{i}$ is mapped to a vertex group of $\mathcal{G}(n+1)$ so is $V_{i}^{\prime}$, hence every nonabelian vertex group of $\operatorname{NPJSJ}(\mathcal{P}(n))$ is as well.

Lemma 8.4 Suppose $L \rightarrow G$ is indecomposable and degenerate, $c_{a}(L)=0$, and $(K, \mathcal{E})<L$ one ended, each $E \in \mathcal{E}$ elliptic in $\operatorname{JSJ}(L)$, and with the induced map $(K, \mathcal{E}) \rightarrow G$ indecomposable. Let $\Delta$ be the decomposition of $K$ induced by the principal cyclic JSJ of $L$. If the image of $K$ in $G$ is contained in a non-QH vertex group of $\operatorname{JSJ}(G)$, then either $\Delta$ is trivial or a nonabelian vertex group of $\Delta$ has abelian image in $G$.

Proof Since $(K, \mathcal{E})$ is freely indecomposable, each vertex/edge group of $\Delta$ is nontrivial. Furthermore, $K$ clearly can't intersect any QH vertex groups of $L$. Since $\Delta$ is induced by the principal cyclic JSJ of $L$ and $c_{a}(L)=0$, each vertex group of $\Delta$ not containing an element of $\mathcal{E}$ is nonabelian or comes from a cyclic vertex group of $L$. Suppose $\Delta$ is nontrivial. By Lemma 8.2 there are two nonabelian vertex groups $V$ and $W$ which are contained in distinct conjugates of vertex groups of $L$. If they are contained in distinct vertex groups of $L$ the proposition is obvious, thus all nonabelian vertex groups of $\Delta$ must be contained in conjugates of some fixed vertex group of $L$, and all other vertex groups are abelian. Since $\Delta$ is induced by the principal cyclic JSJ of $L$, all other vertex groups are infinite cyclic or $K$ intersects a $\mathrm{QH}$ vertex group, but 
if there is an infinite cyclic vertex group then $K$ must intersect two distinct conjugates of vertex groups of $\operatorname{PJSJ}(L)$.

Lemma 8.5 Suppose $P^{\prime}$ is a group with an abelian decomposition $\Delta=\Delta(\mathcal{V}, \mathcal{E}, \mathcal{A})$, $A_{i} \in \mathcal{A}$ noncyclic abelian, with an indecomposable degenerate map $\varphi: P^{\prime} \rightarrow P$, and suppose that the $\varphi\left(A_{i}\right)$ are noncyclic and nonconjugate. Then, all nonabelian vertex groups of $P$ have nonabelian image in $P$, a nonabelian vertex group of $P^{\prime}$ has cyclic image in $P$, or one of the following holds:

- $c_{a}(P)>c_{a}\left(P^{\prime}, \Delta\right)$.

- $\quad P$ has an abelian decomposition $\Delta^{\prime}$ with only noncyclic abelian vertex groups, and $\Delta^{\prime}$ has strictly more noncyclic abelian vertex groups than $\Delta$.

- $P$ has an abelian decomposition $\Delta^{\prime}$ with the same number of noncyclic abelian vertex groups, but the total curvature $\left(\kappa_{A}\right)$ of abelian vertex groups is higher than in $P^{\prime}$.

The proof is another definition chase. See Lemma 6.6

Proof Let $\Delta^{\prime}$ be the decomposition of $P$ obtained by collapsing all edges of $P$ that aren't centralized by the image of some $A_{i}$ or the image of a nonabelian vertex group of $\Delta$ with abelian image. We may assume that $c_{a}(P)=c_{a}\left(P^{\prime}, \Delta\right)$. Under $\boldsymbol{S}_{\mathrm{A}}$, all collapses of subgraphs of groups are of trees, any nonabelian valence one vertex group at any stage of the construction has nonabelian image, and any nonabelian vertex group of $\Delta$ with abelian image has valence at least two. Arguing as in Lemma 6.6, if $\Delta^{\prime}$ doesn't have strictly more abelian vertex groups than $\Delta$, then $\kappa_{A}\left(P^{\prime}\right)>\kappa_{A}(P, \Delta)$.

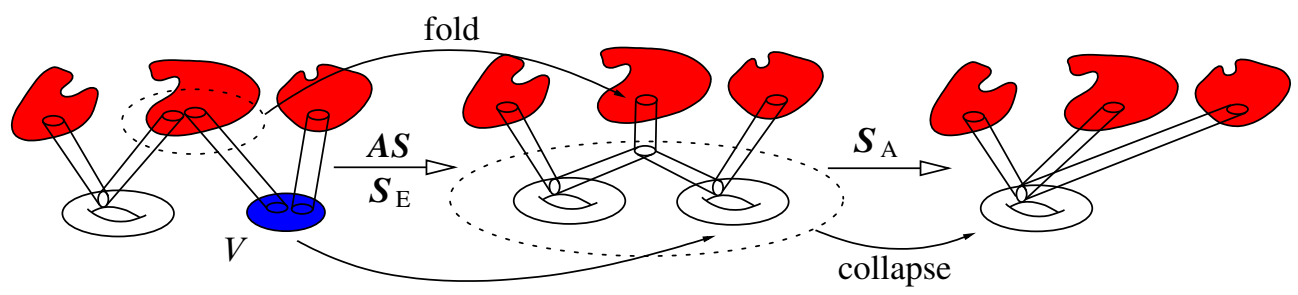

Figure 2: Schematic illustration for Lemma 8.5. If $V$ has abelian image, and if the number of abelian vertex groups does not increase, $\kappa_{A}\left(P^{\prime}\right)>\kappa_{A}(P, \Delta)$.

The first betti numbers of vertex groups of $\boldsymbol{A S}(G)$ are related to the first betti numbers of vertex groups of $\boldsymbol{S}(G)$ : 
Lemma 8.6 Suppose $\varphi: G \rightarrow H$ is degenerate and indecomposable. Let $\Delta$ be a cyclic decomposition of $G$, and suppose that a noncyclic vertex group $G_{v}$ of $\Delta$ with first betti number at least two has cyclic image in $H$. If the image of $G_{v}$ is contained in a small/cyclic abelian vertex group of $H$ then each vertex group in the principal cyclic or quasiprincipal JSJ of $H$ (when defined) has lower first betti number than $H$.

Proof We may assume that $P(A)=A$ for every small abelian vertex group of $H$, otherwise the proposition is obvious. Build $\boldsymbol{A S}(\varphi): \boldsymbol{A S}(G) \rightarrow H$, and collapse all edges of $\boldsymbol{A S}(\Delta)$ not attached to the image of $G_{v}$ to obtain a decomposition $\Delta^{\prime}$. The statement is clearly true for $\boldsymbol{A S}(G)$ with respect to $\Delta^{\prime}$. Each group in the sequence $\left(\boldsymbol{S}_{\mathrm{E}} \circ \boldsymbol{S}_{\mathrm{A}}\right)^{n}(\boldsymbol{A S}(G))$ has a decomposition induced by $\Delta^{\prime}$, and vertex groups of these decompositions are obtained by iteratively adjoining roots to the vertex groups of $\Delta^{\prime}$. Iteratively adjoining roots doesn't increase the first betti number.

Lemma 8.7 (Main lemma) Suppose $\mathcal{L}, \mathcal{G}, \mathcal{R}$, and $\mathcal{P}$ are as in Lemma 8.3. Suppose further that $c_{a}(\mathcal{L}(n))=c_{a}(\mathcal{G}(n))=c_{a}(\mathcal{P}(n))=0$. Then either

$$
\operatorname{Comp}(\mathcal{P} \square \mathcal{L})<\operatorname{Comp}(\mathcal{G} \square \mathcal{L})
$$

or, for each sequence of vertex groups $\mathcal{M}$ of $\operatorname{QPJSJ}(\mathcal{P})$,

$$
\operatorname{Comp}(\mathcal{M} \square \mathcal{L})<\operatorname{Comp}(\mathcal{G} \square \mathcal{L})
$$

Proof Consider the following commutative diagram:

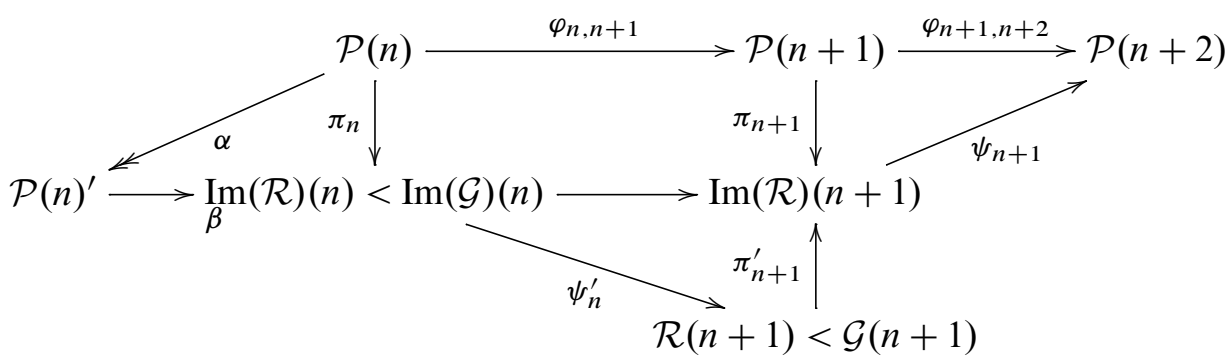

Suppose that $\operatorname{Im}(\mathcal{R}(n))$ is not contained in a vertex group of $\operatorname{PJSJ}(\mathcal{L}(n))$ for any $n$, ie, $\operatorname{Comp}(\mathcal{R} \square \mathcal{L})=\operatorname{Comp}(\mathcal{G} \square \mathcal{L})$. Let $\Delta\left(V_{i}, A_{j}, E_{k}\right)$ be $\operatorname{NPJSJ}(\mathcal{P}(n))$. The edge groups of $\Delta$ have elliptic image in $\mathcal{G}(n), V_{i}$ is indecomposable relative to $\mathcal{E}\left(V_{i}\right)$, thus each $K_{i}:=\operatorname{Im}_{\mathcal{L}(n)}\left(V_{i}\right)$ has inherits a (principal!) decomposition $\Delta_{i}$ over nontrivial cyclic edge groups such that each element of $\operatorname{Im}_{\mathcal{L}(n)}\left(\mathcal{E}\left(V_{i}\right)\right)$ is elliptic. Since $\operatorname{Im}(\mathcal{R}(n))$ is not contained in a vertex group of the principal cyclic JSJ of $\mathcal{L}(n)$ then by Lemma 8.2, without loss, $\Delta_{0}$ is nontrivial and has at least two nonabelian vertex groups contained in distinct conjugates of nonabelian vertex groups of $\operatorname{Im}(\mathcal{G}(n))$. By Lemma 8.3 
$\psi_{n}^{\prime}\left(\operatorname{Im}_{\mathcal{G}(n)}\left(V_{i}\right)\right)$ is contained in a vertex group of $\operatorname{JSJ}(\mathcal{G}(n+1))$, and by Lemma 8.4 some nonabelian vertex group $W$ of $\Delta_{0}$ must have abelian image in $\mathcal{G}(n+1)$. Let $\mathcal{P}(n)^{\prime}$ be the group $\Delta\left(\operatorname{Im}_{\mathcal{L}(n)}\left(V_{i}\right), \operatorname{Im}_{\mathcal{L}(n)}\left(A_{j}\right), \operatorname{Im}_{\mathcal{L}(n)}\left(E_{k}\right)\right)$ with the natural attaching maps. Since the image of each $E_{k}$ in $\mathcal{L}(n)$ is elliptic, the decompositions $\Delta_{i}$ can be used to strictly refine the decomposition $\Delta$ to a decomposition $\Delta^{\prime \prime}$. Since $W$ has abelian image in $\mathcal{G}(n+1)$ it also does in $\mathcal{P}(n+2)$. The decomposition $\Delta^{\prime}$ of $\mathcal{P}(n+2)$ given by Lemma 8.5 is strictly larger than $\operatorname{NPJSJ}(\mathcal{P}(n+2))$ or $c_{a}(\mathcal{P}(n+2))>0$, but $c_{a}(\mathcal{P}(n+2)=0$, which implies that the sequence of abelian vertex group of $\mathcal{P}$ corresponding to the image of $W$ is small, but then $W$ has cyclic image in $\mathcal{P}(n+3)$, hence the vertex groups of $\operatorname{QPJSJ}(\mathcal{P})$ have lower first betti number than $\mathcal{P}$ by Lemma 8.6. If $\mathcal{M}$ is a sequence of vertex groups of $\operatorname{QPJSJ}(\mathcal{P})$ then $\mathrm{b}_{1}(\mathcal{M})<\mathrm{b}_{1}(\mathcal{P})$, hence $\operatorname{Comp}(\mathcal{M} \square \mathcal{L})<\operatorname{Comp}(\mathcal{G} \square \mathcal{L})$.

\section{Adjoining roots}

This section is a generalization of results from earlier work [10]. We are interested in solutions, in the category of limit groups, to equations of the form "adjoin a root to $g$." A tuple

$$
G \stackrel{\nu}{\rightarrow} H \stackrel{\pi}{\rightarrow} G^{\prime}
$$

is an adjunction if $\pi \circ v=\eta$ is injective and $\pi$ is surjective. A staircase is a pair of sequences $(\mathcal{G}, \mathcal{H})$ such that $\mathcal{G}=\mathcal{G}(0) \hookrightarrow \mathcal{G}(1) \hookrightarrow \cdots$ and $\mathcal{G}(i) \rightarrow \mathcal{H}(i+1) \rightarrow \mathcal{G}(i+1)$ is an adjunction.

Definition 9.1 (Adjoining roots) Let $G$ be a finitely generated group, $\mathcal{E}$ a collection of nontrivial abelian subgroups of $G$. For each $E \in \mathcal{E}$, let $\mathcal{F}(E)$ be a collection of finite index abelian supergroups of $E$, with an inclusion map $i_{E, F}: E \hookrightarrow F$ for each $F \in \mathcal{F}(E)$, and let $\mathcal{F}(\mathcal{E})$ be the collection $\{\mathcal{F}(E)\}$. Let

$$
G[\mathcal{F}(\mathcal{E})]:=\left\langle G, F \mid E=i_{E, F}(E)\right\rangle_{F \in \mathcal{F}(E), E \in \mathcal{E}} .
$$

A finitely generated group $H$ is obtained from $G$ by adjoining roots $\mathcal{F}(\mathcal{E})$ to $\mathcal{E}$ if $G<H$ and the inclusion map extends to a surjection $G[\mathcal{F}(\mathcal{E})] \rightarrow H$. Usually the specifics of $\mathcal{F}$ are immaterial, and we omit it from the notation. We always use the term adjunction to refer to adjunctions $G \hookrightarrow H \rightarrow G^{\prime}$ such that $H$ is obtained from $G$ by adjoining roots.

Let $\sim_{Z}$ be the relation 'is conjugate into the centralizer of.' This is an equivalence relation if the group is CSA. CSA groups are commutative transitive and any nontrivial abelian subgroup is contained in a unique maximal abelian subgroup. We may use 
commutative transitivity to simplify systems of adjunctions of roots. Suppose $H$ is obtained from $G$ by adjoining roots $\mathcal{F}(\mathcal{E})$ to $\mathcal{E}$. Let $v$ be the inclusion map. Fix some $\sim_{Z}$ equivalence class $[E]$. By conjugating we may assume that each element of $[E]$ is a subgroup of $Z_{G}(E)$. Replace $[E]$ by $\left\{Z_{G}([E])\right\}$, and replace $\bigcup_{B \in[E]} \mathcal{F}(B)$ by

$$
\left\langle Z_{G}([E]), F \mid B=i_{B, F}(B)\right\rangle_{B \in[E], F \in \mathcal{F}(B)}^{\mathrm{ab}} .
$$

By commutative transitivity, $H$ is a quotient of $G[\mathcal{F}(\mathcal{E})]$. Since limit groups are CSA we make this reduction without comment. Since $\mathcal{F}(E)$ has a single element after this simplification, we will generally use the less ostentatious notation $F(E)$ or just $\sqrt{E}$.

Definition 9.2 (Staircase) We always use the term staircase to indicate a staircase equipped with a family $\mathcal{E}$ of subgroups $\mathcal{E}_{i}$ of $\mathcal{G}(i),(\mathcal{G}, \mathcal{H}, \mathcal{E})$, such that:

- $\mathcal{G}(i-1) \hookrightarrow \mathcal{H}(i) \rightarrow \mathcal{G}(i)$ is an adjunction; $\mathcal{H}(i)$ is obtained from $\mathcal{G}(i-1)$ by adjoining roots to $\mathcal{E}_{i-1}$.

- Each $E^{\prime} \in \mathcal{E}_{i}$ in $\mathcal{G}(i)$ centralizes, up to conjugacy, the image of an element $E$ of $\mathcal{E}_{i-1}$. If $E \in \mathcal{E}_{i-1}$ is mapped to $E^{\prime} \in \mathcal{E}_{i}$ then we require that the image of $Z_{G}(E)$ in $Z_{G^{\prime}}\left(E^{\prime}\right)$ be of finite index.

To fix notation, the maps $\mathcal{G}(i) \hookrightarrow \mathcal{G}(i+1), \mathcal{G}(i) \hookrightarrow \mathcal{H}(i+1)$, and $\mathcal{H}(i+1) \rightarrow \mathcal{G}(i+1)$ are denoted by $\eta_{i}, v_{i}$, and $\pi_{i+1}$, respectively. The length of $\mathcal{G}$ is denoted $\|\mathcal{G}\|$.

A staircase is

- decomposable if all $\mathcal{G}(i)$ are freely decomposable,

- indecomposable if all $\mathcal{G}(i)$ are freely indecomposable,

- $\mathrm{QH}-$ free if no $\mathcal{G}(i)$ has a $\mathrm{QH}$ subgroup,

- mixed if it has both decomposable and indecomposable segments, or, if indecomposable, has QH-free and non-QH-free segments. Otherwise it is unmixed.

Definition 9.3 Let $\left(i_{j}\right)$ be a strictly increasing sequence of indices. A staircase $(\mathcal{V}, \mathcal{W})$, such that $\mathcal{V}(j)=\mathcal{G}\left(i_{j}\right)$ and $\mathcal{W}(j)=\mathcal{H}\left(i_{j}\right)$, with maps obtained by composing maps from $(\mathcal{G}, \mathcal{H}, \mathcal{E})$, is a contraction of $(\mathcal{G}, \mathcal{H}, \mathcal{E})$. A segment of a staircase is a contraction whose indices are consecutive.

To see that a contraction of a staircase is a staircase consider Figure 3 . Each $E \in \mathcal{E}_{i}$ has finite index image in its counterpart in $\mathcal{E}_{i+1}$. Enlarging an abelian group by finite index abelian supergroups multiple times can be done in one step. 


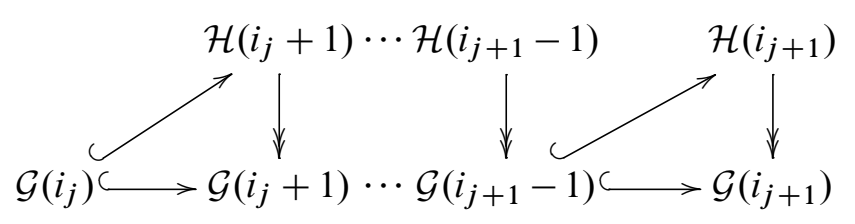

Figure 3: A contraction of a staircase is a staircase.

Recall that the depth of a limit group is the number of levels in its principal cyclic analysis lattice. The depth of a staircase $(\mathcal{G}, \mathcal{H}, \mathcal{E})$ is

$$
\operatorname{depth}((\mathcal{G}, \mathcal{H}, \mathcal{E})):=\max \{\operatorname{depth}(\mathcal{H}(i))\}
$$

The first betti number of $(\mathcal{G}, \mathcal{H}, \mathcal{E})$ is the first betti number of $\mathcal{G}(1)$.

Let $\mathcal{E}$ be a collection of abelian subgroups of a CSA group $G$. We denote by $\|\mathcal{E}\|$ the number of $\sim_{Z}$ equivalence classes in $\mathcal{E}$. The complexity of $(\mathcal{G}, \mathcal{H}, \mathcal{E})$ is the triple

$$
\operatorname{Comp}((\mathcal{G}, \mathcal{H}, \mathcal{E})):=\left(\mathrm{b}_{1}(\mathcal{G}), \operatorname{depth}(\mathcal{H}),\|\mathcal{E}\|\right) .
$$

Complexities are not compared lexicographically: $\left(b^{\prime}, d^{\prime}, e^{\prime}\right) \leq(b, d, e)$ if $b^{\prime} \leq b$, $d^{\prime} \leq d$, and $e^{\prime} \leq e+2 b$. That this defines a partial order follows easily from the definition. The partial order $\leq$ is locally finite. ${ }^{4}$

Let $(\mathcal{G}, \mathcal{H}, \mathcal{E})$ be a staircase. The quantity $\operatorname{NInj}((\mathcal{G}, \mathcal{H}, \mathcal{E}))$ is the number of indices $i$ such that $\mathcal{H}(i) \rightarrow \mathcal{G}(i)$ is not an isomorphism.

Theorem 9.4 Let $(\mathcal{G}, \mathcal{H}, \mathcal{E})$ be a staircase. There is a function

$$
\operatorname{NInj}(\operatorname{Comp}((\mathcal{G}, \mathcal{H}, \mathcal{E})))
$$

such that

$$
\operatorname{NInj}((\mathcal{G}, \mathcal{H}, \mathcal{E})) \leq \operatorname{NInj}(\operatorname{Comp}((\mathcal{G}, \mathcal{H}, \mathcal{E}))) .
$$

Question 2 Is there a complexity that doesn't increase under inclusions, such that if, in an adjunction $G \hookrightarrow H \rightarrow G^{\prime}$, complexity does not decrease, then $H \rightarrow G^{\prime}$ is an isomorphism?

\subsection{Aligning JSJ decompositions of staircases}

Let $G$ be a finitely generated group acting on a simplicial tree $T$ minimally and without inversions. It is a standard fact that the quotient $T / G$ is the underlying graph of a graph of groups decomposition of $G$. If $H<G$ is a finitely generated subgroup, there is a minimal subtree $S \subset T$ fixed (setwise) by $H$, and the action of $H$ on $S$

${ }^{4}\{x \mid a \leq x \leq b\}$ is finite. 
endows $H$ with a graph of groups decomposition. Additionally, there is an induced map of quotient graphs $S / H \rightarrow T / G$. Suppose $G$ and $H$ are freely indecomposable limit groups, $T$ the Bass-Serre tree corresponding to the principal cyclic JSJ of $H$. We say that $G$ and $H$ are aligned if $S / H \rightarrow T / G$ is an isomorphism of graphs and $S / H$ is the underlying graph of the principal cyclic JSJ of $H$.

If $G^{\mathrm{ab}} \rightarrow H^{\mathrm{ab}}$ is virtually onto there is a monotone complexity, with equality implying alignment. The properties of the alignment are then used to construct graphs of spaces and maps between them which resemble Stallings' immersions. The main idea is that an inclusion must either "tighten up" the Grushko or JSJ or it can be written as a map of graphs of groups in a simple way.

Let $T$ be the Bass-Serre tree corresponding to $\operatorname{PJSJ}(H)$, and let $S$ be the minimal subtree for $G$. The quotient $S / G$ is finite and it follows from the definitions that the induced graphs of groups decomposition of $G$ is principal. For convenience, we usually conflate underlying graphs and graphs of groups decompositions. Let $\Delta_{H}=T / H$ and $\Delta_{G}=S / G$ be the underlying graphs, and let $\eta_{\#}$ be the induced map. We label a vertex $v$ of $\Delta_{G}$ by the corresponding label on $\eta_{\#}(v)$, unless $G_{v}$ is abelian, in which case we label it abelian anyway. The map $\eta_{\#}$ is well behaved:

- If $G_{v}$ is nonabelian then the edge groups adjacent to $v$ have nonconjugate centralizers in $G_{v}$.

- Let $B$ be a maximal connected subgraph of $\Delta_{G}$ such that every vertex is abelian. Commutative transitivity implies that $G_{B}$ is abelian, and the fact that all noncyclic abelian subgroups of $H$ are elliptic in $T_{H}$ implies that $B$ is a tree.

- If $v$ is abelian and $\eta_{\#}(v)$ is nonabelian, then $\eta_{\#}(v)$ is rigid.

- A valence one cyclic $v$ is adjacent to a $\mathrm{QH} w$. This follows from the fact that the only edge groups of $H$ not necessarily closed under taking roots are adjacent to $\mathrm{QH}$ vertices, otherwise roots can be absorbed into adjacent abelian or rigid vertices by pulling.

Lemma 9.5 Let $\eta: G \rightarrow H$ be a homomorphism of freely indecomposable limit groups such that $H^{1}(H, \mathbb{Z}) \rightarrow H^{1}(G, \mathbb{Z})$ is injective. Then $G$ is hyperbolic in every essential one-edged abelian splitting of $H$.

If $R$ is a nonabelian vertex group of a GAD $\Delta_{H}$ of $H$, then $G$ intersects a conjugate of $R$ in a nonabelian subgroup. If $\Delta_{G}$ is the induced decomposition of $G$, and there is only one nonabelian vertex group $R^{\prime}$ of $\Delta_{G}$ mapping to $R$, then the map on underlying graphs is a submersion (the induced map on links of vertices is surjective) at $R^{\prime}$. 
Proof If $G$ acts elliptically in some essential one-edged splitting, we claim there is a map $H \rightarrow \mathbb{Z}$ which kills $G$. If the one-edged splitting is an HNN extension the claim is clear. If not, then both vertex groups of the amalgam have a map onto $\mathbb{Z}$ which kills the incident edge group.

To see the second half, suppose not, and let $\Delta_{H}^{\prime}$ be the decomposition of $H$ obtained by conjugating edge maps to $R$ so that all incident edges either have the same or nonconjugate centralizers, and folding together edges of the conjugated decomposition having the same centralizer. Then pull all the centralizers of incident edge groups across the edge they centralize. If $T$ is the tree for $\Delta_{H}^{\prime}$, and $S$ is the minimal $G$-invariant subtree, then the map $S / G \rightarrow T / H$ misses the vertex corresponding to $R$. Let $\Delta^{\prime \prime}$ be the decomposition of $H$ obtained by collapsing all edges not adjacent to $R$. Then $G$ is elliptic in $\Delta^{\prime \prime}$. The first part provides a contradiction.

If the map is not a submersion on $\Delta_{H}$, then the of graphs of groups $\Delta_{G}^{\prime} \rightarrow \Delta_{H}^{\prime}$ is not a submersion at $R$ either, and there is an edge incident to $R$ missed by $\Delta_{G}^{\prime}$. This edge represents an essential splitting of $H$, contradicting the fact that $G$ is hyperbolic in every one edged splitting of $H$.

Definition 9.6 (Complexity of JSJs) Let $G$ be a finitely generated freely indecomposable limit group with a principal cyclic decomposition

$$
G=\Delta(\mathcal{R}, \mathcal{Q}, \mathcal{A}, \mathcal{E})
$$

Let

- $c_{q}(G):=\left|\sum_{Q \in \mathcal{Q}} \chi(Q)\right|$ be the total Euler characteristic of QH subgroups.

- $c_{b q}(G):=\sum_{Q \in \mathcal{Q}} \# \partial Q$ be the total number of boundary components of $\mathrm{QH}$ vertex groups.

- $\mathcal{Z}(G)$ be the collection of conjugacy classes of centralizers of edge groups of $G$.

- $v(R)$ be the valence of $R$ for a rigid vertex $R \in \mathcal{R}$. This is the same as the number of conjugacy classes of centralizers of incident edge groups in $R$.

- $c_{a}(G):=\sum_{A \in \mathcal{A}}(\operatorname{rk}(A)-1)$.

- $c_{b}(G)=c_{a}(G)+\mathrm{b}_{1}(\Delta)$.

The complexity of $G$ with respect to $\Delta$ is the ordered tuple

$$
\operatorname{JComp}(G, \Delta)=\left(c_{q}(G),-c_{b q}(G),|\mathcal{Z}|, c_{b}(G), \mathrm{b}_{1}(\Delta),|\mathcal{R}|, \sum_{R \in \mathcal{R}} v(R)\right) .
$$

We suppress ", $\Delta$ " from the notation if $\Delta$ is the principal cyclic JSJ of $G$. 
Complexities are compared lexicographically. The complexity $\mathrm{JComp}_{i}$ is the restriction of JComp to the first $i$ coordinates.

Throughout this section we assume that $G$ and $H$ are freely indecomposable limit groups, $\eta: G \hookrightarrow H$, and $\eta^{\#}: H^{1}(H, \mathbb{Z}) \rightarrow H^{1}(G, \mathbb{Z})$ is injective.

Lemma 9.7 Let $G$ be a freely indecomposable limit group with principal cyclic JSJ $\Delta_{G}$, let $\mathcal{E}$ be a fixed family of subgroups of $G$, and let $\Delta$ be the principal cyclic decomposition of $G$ associated to the family of principal cyclic splittings in which each $E \in \mathcal{E}$ is elliptic. Then

$$
\operatorname{JComp}(G, \Delta) \leq \operatorname{JComp}(G) \text {, }
$$

and $\operatorname{JComp}(G, \Delta)=\operatorname{JComp}(G)$ if and only if $\Delta$ is the principal cyclic JSJ.

Proof As before, we can make $\Delta$ by cutting QH vertex groups of $\Delta_{G}$ along simple closed curves, folding, and collapsing subgraphs. To handle $c_{b}$, observe that any collection of disjoint simple closed curves on QH vertex groups of $\Delta$ can be completed to a collection which achieves at most $c_{b}(G)$.

The inequalities on $c_{q}$ and $c_{b q}$ are obvious, and if they are equal, then the identity map simply identifies $\mathrm{QH}$ vertex groups. The remaining inequalities are obvious.

Lemma 9.8 $c_{q}(G) \geq c_{q}(H)$. If equality holds then $c_{b q}(G) \leq c_{b q}(H)$.

Proof Let $T$ be the Bass-Serre tree for the restricted JSJ (see Definition 2.3) of $H$. Since $\eta$ is injective, $G$ inherits a graph of groups decomposition $\Delta$ from its action on $T$. Let $Q$ be a vertex group of $\Delta$ conjugate into some element $Q^{\prime}$ of $\mathcal{Q}(H)$. There are two possibilities: $Q$ either has finite or infinite index in $Q^{\prime}$. If $Q$ has infinite index and is nontrivial then $G$ must be freely decomposable, contrary to hypothesis. Thus $Q$ is either trivial or finite index.

Let $c$ be a simple closed curve on some element $Q^{\prime}$ of $\mathcal{Q}(H)$ giving a essential oneedged splitting $\Delta_{c}$ of $H$. By Lemma $9.5 G$ acts hyperbolically in $\Delta_{c}$, hence there is some $Q$ which maps to a finite index subgroup of a conjugate of $Q^{\prime}$. The graph of groups decomposition $\Delta$ of $G$ is obtained by slicing $\mathrm{QH}$ vertex groups of $G$ along simple closed curves, folding, and collapsing subgraphs of the resulting decomposition. This immediately gives $c_{q}(G) \geq c_{q}(H)$.

Suppose $\operatorname{JComp}(G, \Delta)=\operatorname{JComp}(G)$. Let $c_{k}$ be the simple closed curves cutting the QH vertex groups of $\operatorname{PJSJ}(G)$, and let $Q_{1}^{\prime}, \ldots, Q_{m}^{\prime}$ be the complementary components which don't map to QH vertex groups of $H$. Since $c_{q}(G)=c_{q}(H)$, each component $Q_{j}^{\prime}$ has Euler characteristic 0 . Any such complementary component cannot 
be boundary parallel, thus if there are any then $c_{b q}(H)>c_{b q}(G)$. If equality holds then the QH subgroups of $G$ and those of $H$ are in one-to-one correspondence and the associated maps are isomorphisms.

An inclusion $G \hookrightarrow H$ as above is $\mathrm{QH}-$ preserving if it is a one-to-one correspondence on the set of QH vertex groups and the maps are isomorphisms. If $H$ has an inessential one-edged splitting $\Delta$, then $\Delta$ corresponds to an edge connecting a valence one cyclic vertex group of $\operatorname{PJSJ}(H)$ to a $\mathrm{QH}$ vertex group. If $G \hookrightarrow H$ is $\mathrm{QH}$-preserving then it is necessarily bijective on such valence one vertex groups.

It follows immediately from Lemma 9.5 that if $G \hookrightarrow H$ is $\mathrm{QH}-$ preserving then $|\mathcal{Z}(G)| \geq|\mathcal{Z}(H)|$.

Lemma 9.9 $\operatorname{JComp}_{5}(G) \geq \operatorname{JComp}_{5}(H)$. If equality holds then there is an induced bijection $\mathcal{A}(G) \rightarrow \mathcal{A}(H)$, and for each $A, A / P(A) \rightarrow \eta_{\#}(A) / P\left(\eta_{\#}(A)\right)$ is virtually onto.

Proof We first handle $c_{b}$.

Let $\Delta_{H}$ be the principal cyclic JSJ of $H$, and let $\Delta_{G}$ be the decomposition $G$ inherits from its action on $T_{\Delta_{H}}$. We may assume that $G \hookrightarrow H$ is QH-preserving, is bijective on conjugacy classes of centralizers of edge groups. Let

$$
H_{1}\left(H ; \Delta_{H}^{(0)} \backslash \mathcal{A}(H)\right)=H_{1}\left(\Delta_{H}\right) \oplus \bigoplus_{A \in \mathcal{A}(H)} A / P(A) .
$$

Similarly, define $H_{1}\left(G ; \Delta_{G}^{(0)} \backslash \mathcal{A}(G)\right)$. The composition

$$
G \rightarrow H \rightarrow H_{1}\left(H ; \Delta_{H}^{(0)} \backslash \mathcal{A}(H)\right)
$$

factors through $H_{1}\left(G ; \Delta_{G}^{(0)} \backslash \mathcal{A}(G)\right)$. Since $H^{1}(H, \mathbb{Z}) \hookrightarrow H^{1}(G, \mathbb{Z})$ the map

$$
H_{1}\left(G ; \Delta_{G}^{(0)} \backslash \mathcal{A}(G)\right) \rightarrow H_{1}\left(H ; \Delta_{H}^{(0)} \backslash \mathcal{A}(H)\right)
$$

must be virtually onto. But the rank of $\left(H_{1}\left(H ; \Delta_{H}^{(0)} \backslash \mathcal{A}(H)\right)\right)$ is $c_{b}(H)$ and the rank of $\left(H_{1}\left(G ; \Delta_{G}^{(0)} \backslash \mathcal{A}(G)\right)\right)$ is at most $c_{b}(G)$.

Let $\Delta$ be an essential one-edged splitting of $H$ in which all QH subgroups are elliptic. Let $T$ be the corresponding Bass-Serre tree. By Lemma $9.5 G$ doesn't fix a point in $T$ and it inherits an essential splitting $\Delta^{\prime}$ from this action. Since $\eta$ is bijective of the sets of QH subgroups, and restricts to isomorphisms between them, every $\mathrm{QH}$ vertex group of $G$ acts elliptically in $T$. Thus there is an edge group $E^{\prime}$ of $\operatorname{JSJ}(G)$ which maps to a conjugate of the edge group of $\Delta$. Furthermore, $E^{\prime}$ is an essential splitting, otherwise $G$ acts elliptically in $\Delta$. 
Let $A \in \mathcal{A}(G)$ be a noncyclic abelian vertex group of $\operatorname{PJSJ}(G)$. If no element of $\mathcal{A}(H)$ contains the image of $A$, then $c_{b}(G)>c_{b}(H)$. If equality holds there is a well defined map $\mathcal{A}(G) \rightarrow \mathcal{A}(H)$.

Let $A$ be an abelian vertex group of $G$, and $\eta_{\#}(A)$ the associated vertex group of $H$. Since $H^{1}(H) \rightarrow H^{1}(G)$ is injective, the map

$$
A / P(A) \oplus H_{1}\left(\Gamma_{G}\right) \rightarrow \eta_{\#}(A) / P\left(\eta_{\#}(A)\right) \oplus H_{1}\left(\Gamma_{H}\right)
$$

must be virtually onto. This map sends $A / P(A)$ to $\eta_{\#}(A) / P\left(\eta_{\#}(A)\right)$ hence $\mathrm{b}_{1}\left(\Delta_{G}\right) \geq$ $\mathrm{b}_{1}\left(\Delta_{H}\right)$, and if $\mathrm{b}_{1}\left(\Delta_{G}\right)=\mathrm{b}_{1}\left(\Delta_{H}\right)$ then $A / P(A) \rightarrow \eta_{\#}(A) / P\left(\eta_{\#}(A)\right)$ must be virtually onto.

Theorem 9.10 $\operatorname{JComp}(G) \geq \operatorname{JComp}(H)$. If $\eta: G \hookrightarrow H$ and $\operatorname{JComp}(G)=\operatorname{JComp}(H)$, then $\eta$ is bijective on the sets of vertex and edge groups, maps abelian vertex, edge, and peripheral subgroups to finite index subgroups of their respective images. The map from the underlying graph of the principal cyclic JSJ of $G$ to the underlying graph of the principal cyclic JSJ of $H$ is an isomorphism.

The number of values the complexity can take is controlled by $b_{1}$.

Proof of Theorem 9.10 Assume $\operatorname{JComp}_{5}(G)=\operatorname{JComp}_{5}(H)$. By Lemma 9.9, the inclusion is a one-to-one correspondence between noncyclic abelian vertex groups.

Let $\Delta_{H}$ be the principal cyclic JSJ of $H$, let $\eta_{\#}: \Delta_{G} \rightarrow \Delta_{H}$ be the induced map of underlying graphs, and let $R$ be a nonabelian non-QH vertex group of $\Delta_{H}$. By Lemma 9.5 there is a nonabelian vertex group of $\Delta_{G}$ which maps to $R$. Since $\eta$ is bijective on QH subgroups, there is a rigid vertex group $R^{\prime}$ of $G$ which maps to $R$. If $\mathrm{JComp}_{5}(G)=\mathrm{JComp}_{5}(H)$ then $R^{\prime}$ is the unique such vertex group.

Again, by Lemma 9.5, since there is only one vertex group $R^{\prime}$ mapping to $R$, the map $\mathcal{E}\left(R^{\prime}\right) \rightarrow \mathcal{E}(R)$ is onto and $v\left(R^{\prime}\right) \geq v(R)$.

Let $Z$ be an essential cyclic abelian vertex group of $\Delta_{H}$, and let $Z_{1}, \ldots, Z_{k}$ be the vertex groups of $\Delta_{G}$ mapping to $Z$. Since $\eta$ is bijective on nonabelian vertex groups, and since all vertex groups adjacent to $Z$ are nonabelian, the induced map $\eta_{\#}: \sqcup \mathcal{E}\left(Z_{i}\right) \rightarrow \mathcal{E}(Z)$ is bijective. Arguing as in Lemma 9.5, $k=1$ and the map $\mathcal{E}\left(Z_{1}\right) \rightarrow \mathcal{E}(Z)$ is bijective. The same observation shows that if $A$ is noncyclic abelian, then there is a unique abelian vertex group $A^{\prime}$ mapping to $A$ and the associated map of links is onto. The map is also injective, again because $\eta_{\#}$ is a bijective on nonabelian vertex groups. 
Thus, if the complexities are equal, then the inclusion must induce a homeomorphism of underlying graphs. By construction, the map is label preserving, and it automatically respects all incidence and conjugacy data from the respective JSJ decompositions.

This shows that $\operatorname{JComp}\left(G, \Delta_{G}\right) \geq \operatorname{JComp}(H)$, and if equality holds, then the morphism $\Delta_{G} \rightarrow \Delta_{H}$ is an isomorphism of graphs. By Lemma 9.7 $\operatorname{JComp}(G) \geq \operatorname{JComp}\left(G, \Delta_{G}\right)$, and if $\operatorname{JComp}(G)=\operatorname{JComp}(H)$, then $\Delta_{G}$ is just the principal cyclic JSJ of $G$. This gives the first half of the theorem.

Lemma 2.27 bounds the number of vertex groups in the JSJ decomposition. Adjoining roots doesn't increase the first betti number, so if $b_{1}$ and $b_{2}$ are boundary components of a $\mathrm{QH}$ vertex group adjacent to inessential vertex groups, then a simple closed curve cutting off a pair of pants with $b_{1}, b_{2}$ as the two other boundary components makes a contribution of one to $\mathrm{b}_{1}(G) ; n$ nonintersecting simple closed curves make a contribution of $n$ to $\mathrm{b}_{1}(G)$, thus each $\mathrm{QH}$ vertex group is attached to at most $2 \mathrm{~b}_{1}(G)$ inessential vertex groups. Since $\mathrm{b}_{1}(G)$ controls the number of $\mathrm{QH}$ vertex groups, there are boundedly many inessential abelian vertex groups.

If $G \hookrightarrow H$ is as above and $\operatorname{JComp}(G)=\operatorname{JComp}(H)$, then we say that $G$ and $H$ are aligned. Before representing injections of limit groups topologically, we devote a section to proving Theorem 9.4, assuming the results of Section 9.3.

\subsection{Proof of Theorem 9.4}

The bound implicitly computed in the proof of Theorem 9.4 can be made slightly better if we show that nonabelian limit groups with first betti number 2 are free. The next lemma is not necessary, but we record it here for lack of a better place to put it.

Lemma 9.11 Let $G$ be a limit group with first betti number 2 . Then $G \cong \mathbb{F}_{2}$ or $\mathbb{Z}^{2}$.

Proof We may assume $G$ is nonabelian and freely indecomposable. If $G$ is abelian it satisfies the theorem trivially, and if freely decomposable, the free factors are limit groups with first betti number one, and must be infinite cyclic.

The proof is by induction on the depth of $G$. All essential cyclic splittings of $G$ are HNN extensions, otherwise there is a one-edged cyclic splitting such that each vertex group has betti number at least two, and $G$ therefore has first betti number at least three. By Theorem 2.25 the depth of $G$ is finite. Suppose that $G$ has a $\mathrm{QH}$ vertex group $Q$. Then any essential simple closed curve on $Q$ must correspond to an HNN extension of $G$ : $G=G^{\prime} *_{E}$. Since the splitting comes from a $\mathrm{QH}$ vertex group, $G^{\prime}$ must be freely decomposable, hence is $\mathbb{F}_{2}$. If $G$ has no $\mathrm{QH}$ vertex groups the underlying 
graph of its principal cyclic JSJ decomposition must be a bouquet of circles. Let $G=G_{0}>G_{1}>G_{2}>\cdots>G_{n}$ be a sequence of vertex groups of principal cyclic JSJ decompositions such that $G_{i}, i<n-1$, is freely indecomposable and has a bouquet of circles as its principal cyclic JSJ, terminating at the first index $n$ such that such that $G_{n}$ is freely decomposable, hence free. This chain must have finite length since the analysis lattice is finite. We argue that $G_{n}$ free implies that $G_{n-1}$ is free.

Let $f: G_{n-1} \rightarrow \mathbb{F}$ be a homomorphism such that $f\left(G_{n}\right)$ has nonabelian image. Since $G_{n-1}$ is an HNN extension of $G_{n}$, by [10, Theorem 1.5], the images of the incident edge groups in $G_{n}$ can be conjugated to a basis for $G_{n}$ and $G_{n-1}$ is freely decomposable, contrary to hypothesis.

Definition 9.12 (Extension) An extension of an unmixed staircase $(\mathcal{G}, \mathcal{H})$ is a staircase $\left(\mathcal{G}, \mathcal{H}^{\prime}\right)$ such that the diagrams in Figure 4 commute. An extension is admissible if one of the following mutually exclusive conditions holds.

- $\mathcal{G}$ is freely decomposable, and the freely indecomposable free factors of $\mathcal{H}^{\prime}(i)$ embed in $\mathcal{H}(i)$ under $\sigma_{i}$.

- $\mathcal{G}$ is freely indecomposable, has $\mathrm{QH}$ subgroups, and the non-QH vertex groups of restricted JSJ of $\mathcal{H}^{\prime}(i)$ are embedded in $\mathcal{H}(i)$ for all $i$.

- $\mathcal{G}$ is freely indecomposable, $\mathrm{QH}-$ free, and for all $i$, vertex groups of the (restricted) principal cyclic JSJ of $\mathcal{H}^{\prime}(i)$ embed in vertex groups of $\mathcal{H}(i)$ under $\sigma_{i}$.

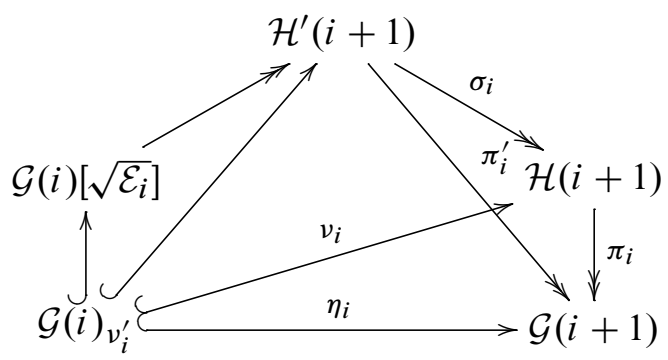

Figure 4: Extensions of sequences

An admissible extension has the property that each $\sigma_{i}$ is strict, surjective, and maps elliptic subgroups of a decomposition of $\mathcal{H}^{\prime}(i+1)$ into $\mathcal{H}(i+1)$. The relation "maps onto" partially orders the collection of extensions, and if, for some $i, \sigma_{i}$ is not one-toone on the sets of vertex groups or edge groups then the extension is nontrivial. An extension is degenerate if there are no contractions admitting nontrivial extensions. The envelope of a rigid vertex group in the principal cyclic JSJ is just the vertex group 
itself, hence $\sigma_{i}$ is an isomorphism if it is a bijection of vertex and edge groups. If $\left(\mathcal{G}, \mathcal{H}^{\prime \prime}\right) \rightarrow\left(\mathcal{G}, \mathcal{H}^{\prime}\right) \rightarrow(\mathcal{G}, \mathcal{H})$ is a pair of admissible extensions then $\left(\mathcal{G}, \mathcal{H}^{\prime \prime}\right) \rightarrow(\mathcal{G}, \mathcal{H})$ is also an admissible extension.

We work with degenerate staircases, but to do so we have to pay a small penalty.

Lemma 9.13 There exists $C=C(K, \operatorname{Comp}())$ such that if $(\mathcal{G}, \mathcal{H}, \mathcal{E})$ is a staircase and $\operatorname{NInj}(\operatorname{Comp}((\mathcal{G}, \mathcal{H}, \mathcal{E}))) \geq C(K, \operatorname{Comp}((\mathcal{G}, \mathcal{H}, \mathcal{E})))$, then there is a degenerate extension of a contraction $\left(\mathcal{G}^{\prime}, \mathcal{H}^{\prime}, \mathcal{E}^{\prime}\right)$ of $(\mathcal{G}, \mathcal{H}, \mathcal{E})$ with $\operatorname{NInj}\left(\left(\mathcal{G}^{\prime}, \mathcal{H}^{\prime}, \mathcal{E}^{\prime}\right)\right) \geq K$ and $\operatorname{Comp}\left(\left(\mathcal{G}^{\prime}, \mathcal{H}^{\prime}, \mathcal{E}^{\prime}\right)\right) \leq \operatorname{Comp}((\mathcal{G}, \mathcal{H}, \mathcal{E}))$.

The constants in this lemma do not depend on $\|\mathcal{E}\|$. The proof is formally identical to the proof of Theorem 4.3. To adapt the proof, we need to show that the strict resolutions arising in an extension have bounded length. This follows from Lemma 2.28, bounding the rank of $\mathcal{H}(i)$ from above by a function of $\operatorname{Comp}((\mathcal{G}, \mathcal{H}))$, but a proof more in the spirit of this section goes as follows: If $\mathcal{H}^{(n)}(i) \rightarrow \cdots \rightarrow \mathcal{H}(i)$ is a strict resolution appearing in a sequence of extensions, then $\operatorname{JComp}(G) \geq \operatorname{JComp}\left(\mathcal{H}^{(m)}(i)\right)$ (see Theorem 9.10 and Definition 9.6), moreover, if $\mathcal{H}^{(m+1)}(i) \rightarrow \mathcal{H}^{(m)}(i)$ is not injective on sets of vertex or edge spaces, or collapses subsurface groups of QH vertex groups, the complexity must decrease. By Theorem 9.10 the number of values the complexity takes is controlled by $\mathrm{b}_{1}$, and the resolutions have length controlled by $\operatorname{Comp}((\mathcal{G}, \mathcal{H}, \mathcal{E}))$.

In all unmixed cases the strategy of proof is the same: either there is compatibility between (collapses of) RJSJ/Grushko decompositions, the complexity decreases, or a sequence admits a proper extension.

Definition $9.14\left(\chi_{\mathrm{fd}}\right)$ Let $G$ be a finitely generated group with Grushko decomposition $G=G_{1} * \cdots * G_{p} * \mathbb{F}_{q}$. Define $\chi_{\mathrm{fd}}(G):=(q-1, p)$.

The number of values $\chi_{\mathrm{fd}}$ can take on groups with $\mathrm{b}_{1}=b$ is bounded by $b^{3}$. The next theorem is [10, Theorem 1.3]

Theorem 9.15 ( $\chi_{\mathrm{fd}}$ and adjoining roots to groups) Suppose that $\phi: G \hookrightarrow H$ and $H$ is a quotient of $G^{\prime}=G\left[\sqrt[k_{i}]{\gamma_{i}}\right], \Gamma_{i}$ a collection of distinct conjugacy classes of indivisible elements of $G$ such that $\Gamma_{i} \neq \Gamma_{j}^{-1}$ for all $i, j$ and $\gamma_{i} \in \Gamma_{i}$. Then $\chi_{\mathrm{fd}}(G) \geq \chi_{\mathrm{fd}}(H)$. If equality holds and $H$ has no $\mathbb{Z}_{2}$ free factors, then there are presentations of $G$ and $H$ as

$$
G \cong G_{1} * \cdots * G_{p} * \mathbb{F}_{q}^{G}, \quad H \cong H_{1} * \cdots * H_{p} * \mathbb{F}_{q}^{H}
$$

a partition of $\left\{\Gamma_{i}\right\}$ into subsets $\Gamma_{j, i}, j=0, \ldots, p, i=1, \ldots, i_{p}$, representatives $\gamma_{j, i} \in G_{j} \cap \Gamma_{j, i}, i \geq 1, \gamma_{0, i} \in \mathbb{F}_{q}^{G} \cap \Gamma_{0, i}$, such that with respect to the presentations of $G$ and $H$ : 
- $\phi\left(G_{i}\right)<H_{i}$.

- $G_{j}\left[\sqrt[k_{j, i}]{\gamma_{j, i}}\right] \rightarrow H_{j}$.

- $\phi\left(\mathbb{F}_{q}^{G}\right)<\mathbb{F}_{q}^{H}$.

- $\mathbb{F}_{q}^{G}=\left\langle\gamma_{0,1}\right\rangle * \cdots *\left\langle\gamma_{0, i_{0}}\right\rangle * F$.

- $\mathbb{F}_{q}^{H}=\left\langle\sqrt{\gamma_{0,1}}\right\rangle * \cdots *\left\langle\sqrt{\gamma_{0, i_{0}}}\right\rangle * F$.

- $G^{\prime} \cong G_{1}\left[\sqrt{\gamma_{1, i}}\right] * \cdots * G_{p}\left[\sqrt{\gamma_{p, i}}\right] *\left\langle\sqrt{\gamma_{0,1}}\right\rangle * \cdots *\left\langle\sqrt{\gamma_{0, i_{0}}}\right\rangle * F$.

All homomorphisms are those suggested by the presentations, and restriction to $F$ is the identity.

Remark 9.16 Theorem 9.15 is stated in terms of adjoining roots to cyclic subgroups of a group, whereas Definition 9.1 refers to collections of abelian subgroups. The complexity \|\| is unchanged by passing from a noncyclic abelian subgroup to cyclic subgroups.

Definition 9.17 (Free products) Let $\left(\mathcal{G}_{i}, \mathcal{H}_{i}\right)$ be a collection of staircases on the same index set $I$. Then the graded free product $\left(\left(*_{i} \mathcal{G}_{i}\right),\left(*_{i} \mathcal{H}_{i}\right)\right)$, with the obvious maps, is also a sequence of adjunctions of roots.

Lemma 9.18 Suppose Theorem 9.4 holds for all staircases with complexity less than $\left(b_{0}, d_{0}, e_{0}\right)$. Then Theorem 9.4 holds for unmixed freely decomposable staircases of complexity $\left(b_{0}, d_{0}, e_{0}\right)$.

Proof Let $(\mathcal{G}, \mathcal{H}, \mathcal{E})$ be a staircase with complexity $\left(b_{0}, d_{0}, e_{0}\right)$. Since limit groups are torsion free, no $\mathcal{G}(i)$ has a $\mathbb{Z}_{2}$ free factor, and by Theorem 9.15 for all but $\mathrm{b}_{1}(\mathcal{G}(1))^{3}$ indices $i_{j}$, the subsequences $\mathcal{G}\left(i_{j}\right) \hookrightarrow \mathcal{G}\left(i_{j}+1\right) \hookrightarrow \cdots \hookrightarrow \mathcal{G}\left(i_{j+1}-1\right)$ can be decomposed into free products of freely indecomposable staircases. Moreover, elements of $\mathcal{E}_{i}$ are either part of a basis of a free factor of $\mathcal{G}(i)$ or are conjugate into a freely indecomposable free factor of $\mathcal{G}(i)$. Write $\mathcal{G}(i)$ as the free product

$$
\mathcal{G}(i)_{1} * \cdots * \mathcal{G}(i)_{p} * F_{i}
$$

given by Theorem 9.15, where $F_{i}$ is a free group of rank $q$ and $\chi_{\mathrm{fd}}(\mathcal{G}(i))=(q-1, p)$ for all $i$. Let $\mathcal{E}_{i}^{j}$ be the subset of $\mathcal{E}_{i}$ consisting of elements conjugate into $\mathcal{G}(i)_{j}$, and rearrange indices so that $\mathcal{G}(i)_{j}$ maps to $\mathcal{G}(i+1)_{j}$ for all $j$. Let $\mathcal{E}_{i}^{0}$ be the elements of $\mathcal{E}$ which are conjugate into $F_{i}$. There are decompositions

$$
\mathcal{G}(i)\left[\sqrt{\mathcal{E}_{i}}\right] \cong\left(\mathcal{G}(i)_{1}\left[\sqrt{\mathcal{E}_{i}^{1}}\right] * \cdots * \mathcal{G}(i)_{p}\left[\sqrt{\mathcal{E}_{i}^{p}}\right]\right) * F_{i}\left[\sqrt{\mathcal{E}_{i}^{0}}\right]
$$


where the last factor is free. Let

$$
\mathcal{H}(i+1)_{j}:=\operatorname{Im}_{\mathcal{H}(i+1)}\left(\mathcal{G}(i)_{j}\left[\sqrt{\mathcal{E}_{i}^{j}}\right]\right) .
$$

The sequence $\mathcal{H}^{\prime}$ defined by

$$
\mathcal{H}^{\prime}(i+1):=\left(*_{j} \mathcal{H}(i+1)_{j}\right) * F_{i}\left[\sqrt{\mathcal{E}_{i}^{0}}\right]
$$

is an extension of $\mathcal{H},\left(\mathcal{G}, \mathcal{H}^{\prime}, \mathcal{E}\right)$ splits as a free product, the freely indecomposable free factors of which are $\left(\mathcal{G}_{j}, \mathcal{H}_{j}^{\prime}, \mathcal{E}^{j}\right)$. These free factors have strictly lower $\mathrm{b}_{1}$ than $\mathcal{G}$, depth at most $d_{0}=\operatorname{depth}(\mathcal{H})$, hence have

$$
\operatorname{NInj}\left(\left(\mathcal{G}_{j}, \mathcal{H}_{j}^{\prime}, \mathcal{E}^{j}\right)\right) \leq \operatorname{NInj}\left(\operatorname{Comp}\left(b_{0}-1, d_{0}, e_{0}\right)\right)=: B .
$$

If $\|\mathcal{G}\|>B \cdot \mathrm{b}_{1}(\mathcal{G}) \geq B \cdot p$, then, for some index $l$, the map $\mathcal{H}^{\prime}(l) \rightarrow \mathcal{G}(l)$ must be an isomorphism. Since this map factors through $\mathcal{H}(l), \mathcal{H}(l) \rightarrow \mathcal{G}(l)$ is an isomorphism as well.

Let $(\mathcal{G}, \mathcal{H}, \mathcal{E})$ be a degenerate staircase of complexity at most $(b, 2, e)$. By the proof of Theorem 9.15, the staircase splits as a free product of freely indecomposable staircases $\left(\mathcal{G}_{i}, \mathcal{H}_{i}, \mathcal{E}^{i}\right)$, each $\mathcal{H}_{i}(j)$ elementary. If $\mathcal{G}_{i}$ is abelian, then $\mathcal{H}_{i}(j) \rightarrow \mathcal{G}_{i}(j)$ is an isomorphism, and if nonabelian, $\mathcal{H}_{i}(j)$ is the fundamental group of a closed surface. Since $\mathcal{G}_{i}$ is freely indecomposable, it is also the fundamental group of a closed surface. Divide $\mathcal{G}_{i}$ into segments such that the Euler characteristic is constant on each segment. Then $\mathcal{G}_{i}(j) \rightarrow \mathcal{G}_{i}(j+1)$ is an isomorphism on each segment and $\mathcal{H}_{i}(j)$ is a trivial extension of $\mathcal{G}_{i}(j-1)$ for all $j$ on each segment, thus $\mathcal{H}_{i}(j) \rightarrow \mathcal{G}_{i}(j)$ is an isomorphism.

The next step is to analyze indecomposable staircases with QH subgroups.

Lemma 9.19 Suppose Theorem 9.4 holds for all staircases with strictly lower complexity than $\left(b_{0}, d_{0}, e_{0}\right)$. Then Theorem 9.4 holds for staircases with $\mathrm{QH}$ subgroups and complexity $\left(b_{0}, d_{0}, e_{0}\right)$.

We find an extension $\left(\mathcal{G}, \mathcal{H}^{\prime}, \mathcal{E}\right)$ of $(\mathcal{G}, \mathcal{H}, \mathcal{E})$ such that the $\mathrm{QH}$ subgroups of $\mathcal{H}^{\prime}$ are exactly the images of those from $\mathcal{G}$. See Figure 5. The group $\mathcal{H}(i)$ is a homomorphic image of a limit group which shares its restricted JSJ with $\mathcal{G}(i)$ and $\mathcal{G}(i+1)$.

The following follows from Sublemma 9.25, the proof of which is postponed until Section 9.3.

Lemma 9.20 Let $G^{\prime}$ be obtained from $G$ by adjoining roots to a collection of abelian subgroups $\mathcal{E}$. If $\operatorname{JComp}(G)=\operatorname{JComp}\left(G^{\prime}\right)$ then every element $E \in \mathcal{E}$ such that $[E: F(E)]>1$ is conjugate into a non-QH vertex group of $\operatorname{RJSJ}(G)$. 
We use the immersion representing $G \hookrightarrow G^{\prime}$ constructed in Section 9.3.1.

Proof Pick $E$ as in the statement of the lemma. We are done if $E$ is elliptic in every one edged splitting of $G$ obtained by cutting a QH subgroup along an essential simple closed curve. Start with an immersion representing the RJSJ decompositions of $G$ and $G^{\prime}$, and let $\Sigma_{Q}$ be the surface containing $c$. There is a unique element $\eta_{\#}(Q)$ containing the image of $Q$, and the map $Q \rightarrow \eta_{\#}(Q)$ is surjective. Since $Q \rightarrow$ $\eta_{\#}(Q)$ is represented by a homeomorphism $\Sigma_{Q} \rightarrow \Sigma_{\eta_{\#}(Q)}$ there is a simple closed curve $\eta_{\#}(c)$ contained in $\Sigma_{\eta_{\#}(Q)}$ and a closed annular neighborhood $A$ of $c$ mapping homeomorphically to a neighborhood of $\eta_{\#}(c)$. Use these neighborhoods to construct graphs of spaces $Y_{G}$ and $Y_{G^{\prime}}$ representing $G$ and $G^{\prime}$ by regarding the annulus as a new edge space and then collapsing all but the newly introduced edges. By construction, the map $Y_{G} \hookrightarrow Y_{G^{\prime}}$ is an immersion. By Sublemma 9.25, if some element of $\mathcal{E}$ crosses $c$, then $c$ maps to a power of $\eta_{\#}(c)$.

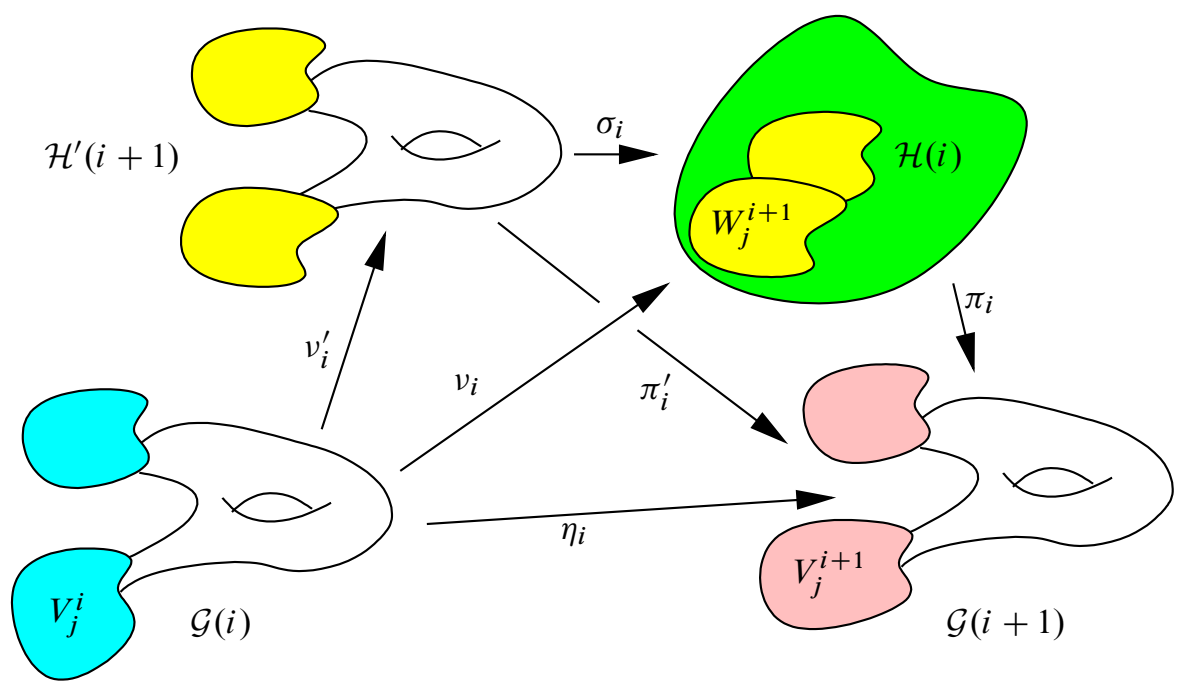

Figure 5: Illustration of Lemma 9.19

Proof of Lemma 9.19 Suppose $(\mathcal{G}, \mathcal{H})$ is a staircase such that $\chi_{\mathrm{fd}}(\mathcal{G}(i))$ is constant, with $c_{q}(\mathcal{G}(1)) \neq 0$. Let $\Delta_{i}$ be the RJSJ of $\mathcal{G}(i)$. Every edge of $\Delta_{i}$ is infinite cyclic and connects a vertex group to a boundary component of a $\mathrm{QH}$ vertex group. Since the inclusions $\mathcal{G}(i) \hookrightarrow \mathcal{G}(i+1)$ respected graphs of spaces, by the first part of Lemma 9.20, every element of $\mathcal{E}_{i}$ is conjugate into some non-QH vertex group of $\Delta_{i}$. Let $V_{1}^{i}, \ldots, V_{n}^{i}$ be the non-QH vertex groups of $\Delta_{i}$. We regard $\mathcal{G}(i)$ as a graph of 
groups $\Gamma\left(V_{j}^{i}, Q_{k}, E_{l}\right)$, where $\mathcal{G}(i) \hookrightarrow \mathcal{G}(i+1)$ is compatible with the decomposition $\Gamma$ : $V_{j}^{i}$ maps to a conjugate of $V_{j}^{i+1}$, the map respects edge group incidences, and the inclusion is the identity on the $\mathrm{QH}$ vertex groups $Q_{k}$.

Let $\mathcal{E}_{i}^{j}$ be the set of elements of $\mathcal{E}_{i}$ conjugate into $V_{j}^{i}$, and arrange, by conjugation, that each $E \in \mathcal{E}_{i}^{j}$ is contained in $V_{j}^{i}$. Let $W_{j}^{i+1}$ be the image of

$$
V_{j}^{i}\left[\sqrt{\mathcal{E}_{i}^{j}}\right]
$$

in $\mathcal{H}(i+1)$ and let $\mathcal{H}^{\prime}(i+1)=\Gamma\left(W_{j}^{i+1}, Q_{k}, E_{l}\right)$. Then $\left(\mathcal{G}, \mathcal{H}^{\prime}, \mathcal{E}\right)$ is an extension of $(\mathcal{G}, \mathcal{H}, \mathcal{E})$ : The obvious map $\sigma_{i}: \mathcal{H}^{\prime}(i) \rightarrow \mathcal{H}(i)$ is strict, therefore $\mathcal{H}^{\prime}$ is a sequence of limit groups. By definition, $V_{j}^{i+1}$ is obtained from $V_{j}^{i}$ by adjoining roots. Let $\mathcal{V}_{j}$ be the sequence $\mathcal{V}_{j}(i)=V_{j}^{i}$ and let $\mathcal{W}_{j}(i)=W_{j}^{i}$.

The staircases $\left(\mathcal{V}_{j}, \mathcal{W}_{j}, \mathcal{E}^{j}\right)$ all have lower first betti number than $(\mathcal{G}, \mathcal{H}, \mathcal{E})$. Let Verts $\left(b_{0}\right)$ be the maximal number of vertex groups in decomposition of limit groups with first betti number $b_{0}$. This is finite by Lemma 2.27. If $\|\mathcal{G}\|>\operatorname{NInj}\left(\left(b_{0}-1, d_{0}, e_{0}\right)\right)$. $\operatorname{Verts}\left(b_{0}\right)$ then for at least one index $l$ all $\mathcal{W}_{j}(l) \rightarrow \mathcal{V}_{j}(l+1)$ are injective. Thus $\pi_{l}^{\prime}$ is RJSJ-strict. Since all modular automorphisms of $\mathcal{H}^{\prime}(l)$ are either inner, Dehn twists in boundary components of $\mathrm{QH}$ vertex groups, or induced by boundary respecting homeomorphisms of surfaces representing QH vertex groups, by construction, every element of $\operatorname{Mod}\left(\mathcal{H}^{\prime}(l), \operatorname{RJSJ}\left(\mathcal{H}^{\prime}(l)\right)\right)$ pushes forward to a modular automorphism of $\mathcal{G}(l)$. By Lemma $2.14 \mathcal{H}^{\prime}(l) \rightarrow \mathcal{G}(l)$ is an isomorphism. Since $\pi_{l}^{\prime}=\pi_{l} \circ \sigma_{l}$, $\pi_{l}$ is an isomorphism.

We now consider indecomposable sequences without QH subgroups. Recall that the neighborhood $\mathcal{N}(V)$ of a vertex group $V$ of a GAD is the subgroup generated by $V$ and conjugates of adjacent non-QH vertex groups intersecting $V$ nontrivially.

Let $G \hookrightarrow H \rightarrow G^{\prime}$ be an adjunction and suppose $G$ is freely indecomposable, has no QH vertex groups, and that $\operatorname{JComp}(G)=\operatorname{JComp}\left(G^{\prime}\right)$. Let $\eta: G \rightarrow G^{\prime}$ be the inclusion map. An abelian vertex group $A$ of $G$ is $H$-elliptic if $H$ doesn't have a principal cyclic splitting over a subgroup of $Z_{H}(v(A))$.

Let $\mathcal{A}_{H}$ be the collection of abelian vertex groups of $G$ which are $H$-elliptic. Suppose that $H$ is obtained from $G$ by adjoining roots to the collection $\mathcal{E}$. Let $\mathcal{E}_{H}^{\text {ell }}$ be the subcollection of $\mathcal{E}$ consisting of elements which are hyperbolic in the principal cyclic JSJ of $G$ but which are elliptic in the principal cyclic JSJ of $H$. Let $\operatorname{JSJ}_{H}(G)$ be the JSJ decomposition of $G$ with respect to the collection of principal cyclic splittings in which all $\mathcal{N}(A), A \in \mathcal{A}_{H}$, and $E \in \mathcal{E}_{H}^{\text {ell }}$ are elliptic:

$$
\operatorname{JSJ}_{H}(G):=\operatorname{JSJ}\left(G ;\left\{\mathcal{N}(A), E \mid A \in \mathcal{A}_{H}, E \in \mathcal{E}_{H}^{\mathrm{ell}}\right\}\right)
$$


Let $\mathrm{JSJ}_{H}^{*}\left(G^{\prime}\right)$ be the JSJ decomposition of $G^{\prime}$ associated to the collection of all principal cyclic splittings of $G^{\prime}$ in which all $\eta_{\#}\left(A_{H}\right), A \in \mathcal{A}_{H}$, and $\eta_{\#}(E), E \in \mathcal{E}_{H}^{\text {ell }}$, are elliptic. That is,

$$
\mathrm{JSJ}_{H}^{*}\left(G^{\prime}\right):=\operatorname{JSJ}\left(G^{\prime} ;\left\{\mathcal{N}\left(\eta_{\#}(A)\right), \eta_{\#}(E) \mid A \in \mathcal{A}_{H}, E \in \mathcal{E}_{H}^{\mathrm{ell}}\right\}\right) .
$$

The idea is that the decompositions of $G$ and $G^{\prime}$ induced by $H$ are closely related to the principal cyclic JSJ of $H$ as long as there are no proper extensions. Let $V$ be a vertex group of $\operatorname{JSJ}_{H}(G)$. There is a vertex group $\eta_{\#}(V)$ of $\operatorname{JSJ}_{H}^{*}\left(G^{\prime}\right)$ contains the image of $V$. Let $\mathcal{E}_{V}$ be the collection of elements of $\mathcal{E}$ which are conjugate into $V$, along with the collection of incident edge groups. Likewise for $\eta_{\#}(V)$, let $\mathcal{E}\left(\eta_{\#}(V)\right)$ be the set of centralizers of images of elements of $\mathcal{E}_{V}$.

Lemma 9.21 Let $G \hookrightarrow H \rightarrow G^{\prime}$ be an adjunction without any proper extensions, and suppose $G$ is freely indecomposable, has no $\mathrm{QH}$ vertex groups, and that $\operatorname{JComp}(G)=$ $\operatorname{JComp}\left(G^{\prime}\right)$. Let $\eta: G \hookrightarrow G^{\prime}$ be the inclusion map. Then the following hold:

- For each vertex group $W$ of the principal cyclic JSJ of $H$ there are unique vertex groups $V$ and $\eta_{\#}(V)$ of $\operatorname{JSJ}_{H}(G)$ and $\mathrm{JSJ}_{H}^{*}\left(G^{\prime}\right)$, respectively, such that $v(V)<W, \pi(W)<\eta_{\#}(V)$.

- $\operatorname{JSJ}_{H}(G)=\operatorname{PJSJ}\left(G, \mathcal{E}_{H}^{\text {ell }}\right), \operatorname{JSJ}_{H}^{*}\left(G^{\prime}\right)=\operatorname{PJSJ}\left(G^{\prime}, \eta_{\#}\left(\mathcal{E}_{H}^{\text {ell }}\right)\right)$.

- $W$ is obtained from $V$ by adjoining roots to $\mathcal{E}_{V}$ and $\left\|\mathcal{E}_{V}\right\| \leq\|\mathcal{E}\|+2 \mathrm{~b}_{1}(G)$.

- $\eta_{\#}(V)$ is obtained from $\pi(W)$ by adjoining roots to the images of $\mathcal{E}(V)$ (the edge groups incident to $V$ ).

- If $\pi$ is injective on vertex groups then it is an isomorphism.

We postpone the proof of Lemma 9.21 until Section 9.3.

9.2.1 Proof of Theorem 9.4 Let $(\mathcal{G}, \mathcal{H})$ be a staircase with complexity $\left(b_{0}, d_{0}, e_{0}\right)$, such that no contraction has any proper extensions, and suppose that Theorem 9.4 holds for staircases of lower complexity. By Theorem 9.10 there is some constant $B\left(b_{0}\right)$ such that $(\mathcal{G}, \mathcal{H})$ can be divided into $B\left(b_{0}\right)$ segments of constant complexity: (Segments are allowed to be empty.)

$$
\begin{gathered}
(\mathcal{G}, \mathcal{H}) \mapsto\left\{\left(\mathcal{G}_{i}, \mathcal{H}_{i}\right)\right\}_{i=1, \ldots, B\left(b_{0}, d_{0}\right)} \\
\mathcal{G}_{i}(1)=\mathcal{G}\left(j_{i}\right), \ldots \quad \mathcal{H}_{i}(2)=\mathcal{H}\left(j_{i}+1\right), \ldots
\end{gathered}
$$

Only the last of these can be indecomposable. Each decomposable staircase $\left(\mathcal{G}_{i}, \mathcal{H}_{i}\right)$, by Lemma 9.18 , has NInj bounded above by $b_{0} \cdot \operatorname{NInj}\left(b_{0}-1, d_{0}, e_{0}\right)$, since there are at most $b_{0}$ freely indecomposable free factors. Thus we may restrict our attention to 
indecomposable staircases. By Theorem 9.10, we may divide the staircase $(\mathcal{G}, \mathcal{H}, \mathcal{E})$ into boundedly many segments, the number depending only on the complexity of $b_{1}(\mathcal{G})$, exhausting the staircase, such that JComp is constant on each segment. By Lemma 9.13 we may assume that each segment is degenerate.

Like the case when each $\mathcal{G}(i)$ is freely decomposable, if $\mathcal{G}(i)$ has a $\mathrm{QH}$ vertex group, by Lemma 9.19 such staircases have bounded NInj.

The only possibility left is that long unmixed segments of $(\mathcal{G}, \mathcal{H})$ are indecomposable and QH-free. Let $I$ be the index set for $\mathcal{G}$, and color the triple $i<j<k$ red if $\operatorname{JSJ}_{\mathcal{H}(j)}^{*}(\mathcal{G}(j)) \cong \operatorname{JSJ}_{\mathcal{H}(k)}(\mathcal{G}(j))$, and blue otherwise. Then by the Ramsey theorem, for all $K$ there exists an $L$ such that if $\|\mathcal{G}\|>L$ then there is a subset $I^{\prime} \subset I$ of size at least $K$ such that all triples whose elements are in $I^{\prime}$ have the same color.

Lemma 9.22 The size of blue subsets is controlled by $b_{1}(\mathcal{G})$ and $\|\mathcal{E}\|$.

Proof Suppose $\left|I^{\prime}\right|>2^{\|\mathcal{E}\|}$, and consider the collection of principal cyclic decompositions $\left\{\operatorname{JSJ}_{\mathcal{H}(l)}(\mathcal{G}(i))\right\}$. Since $\operatorname{JSJ}_{\mathcal{H}(j)}(\mathcal{G}(i))=\operatorname{PJSJ}\left(\mathcal{G}(i), \mathcal{E}^{\prime}\right)$ for some $\mathcal{E}^{\prime} \subset \mathcal{E}_{i}$ and $\operatorname{JSJ}_{\mathcal{H}(k)}(\mathcal{G}(i))=\operatorname{PJSJ}\left(\mathcal{G}(i), \mathcal{E}^{\prime \prime}\right)$ for some $\mathcal{E}^{\prime} \subset \mathcal{E}_{i}$. By the pigeonhole principle, for some $i<j<k$, $\operatorname{JSJ}_{\mathcal{H}(j)}(\mathcal{G}(i))$ and $\operatorname{JSJ}_{\mathcal{H}(k)}(\mathcal{G}(i))$ are equivalent. Then $\mathrm{JSJ}_{\mathcal{H}(k)}(\mathcal{G}(j)) \cong \mathrm{JSJ}_{\mathcal{H}(j)}^{*}(\mathcal{G}(j))$ since a GAD is determined up to equivalence by its elliptic subgroups.

Suppose again that $\left(b_{0}, d_{0}, e_{0}\right)$ is the lowest complexity for which Theorem 9.4 doesn't hold. By Lemma 9.22 and the prior discussion, there must be degenerate indecomposable QH-free staircases $(\mathcal{G}, \mathcal{H}, \mathcal{E})$, complexity $\left(b_{0}, d_{0}, e_{0}\right)$, and arbitrarily high NInj.

Let $(\mathcal{G}, \mathcal{H})$ be such a staircase. Let $\mathcal{V}_{j}^{\prime}(i)$ denote the nonabelian vertex groups of $\operatorname{JSJ}_{\mathcal{H}(i+1)}(\mathcal{G}(i))$, indexed such that $\mathcal{V}_{j}^{\prime}(i)$ maps to $\mathcal{V}_{j}^{\prime}(k)$ for all $k>i$. Let $\mathcal{W}_{j}(i)$ be the corresponding rigid vertex group of $\mathcal{H}(i)$. By the second bullet of Lemma 9.21, $\mathcal{W}_{j}(i+1)$ is obtained from $\mathcal{V}_{j}^{\prime}(i)$ by adjoining roots to $\mathcal{E}_{i}^{j \prime}$, the set of elements of $\mathcal{E}_{i}$ which are conjugate into $\mathcal{V}_{j}^{\prime}(i)$, along with the incident edge groups.

Let $\mathcal{V}_{j}(i)<\mathcal{V}_{j}^{\prime}(i)$ be the image of $\mathcal{W}_{j}(i)$ in $\mathcal{G}(i)$. By the third bullet of Lemma 9.21, $\mathcal{V}_{j}^{\prime}(i)$ is obtained from $\mathcal{V}_{j}(i)$ by adjoining roots to the images of the edge groups incident to $\mathcal{W}_{j}(i)$. Let

$$
\mathcal{E}_{i}^{j}:=\left\{E \cap \mathcal{V}_{j}(i) \mid E \in \mathcal{E}_{i}^{j \prime}\right\} \cup\left\{E \cap \mathcal{V}_{j}(i) \mid E \in \mathcal{E}\left(\mathcal{V}_{j}(i)\right)\right\} .
$$

The incident edge groups are cyclic, and we can build $\mathcal{W}_{j}(i)$ by simply adjoining roots to $\mathcal{E}_{i}^{j}$ in $\mathcal{V}_{j}(i)$. Then $\mathcal{E}_{i}^{j}$ is larger than $\mathcal{E}$ by at most the number of edge groups incident to $\mathcal{V}_{j}^{\prime}(i)$, which is at most $2 \mathrm{~b}_{1}(\mathcal{G})$. That is,

$$
\left\|\mathcal{E}_{i}^{j}\right\| \leq\|\mathcal{E}\|+2 b_{1}(\mathcal{G}) \text {. }
$$


Given a sufficiently long QH-free staircase $(\mathcal{G}, \mathcal{H}, \mathcal{E})$, we passed to a degenerate extension (which we will also call $(\mathcal{G}, \mathcal{H}, \mathcal{E})$ ) of a substaircase of arbitrary prescribed length, such that the sequences of vertex groups $\left(\mathcal{V}_{j}, \mathcal{W}_{j}, \mathcal{E}^{j}\right)$ of the extension were staircases as well. The vertex groups of the extension are subgroups of the vertex groups of $\mathcal{H}$, hence the depth of $\mathcal{W}_{j}(i)$ is strictly less than the depth of $\mathcal{H}$. Since the first betti number of $\mathcal{W}_{j}(i)$ is at most $b_{1}(\mathcal{H}) \operatorname{Comp}((\mathcal{G}, \mathcal{H}, \mathcal{E}))>\operatorname{Comp}\left(\left(\mathcal{V}_{j}, \mathcal{W}_{j}, \mathcal{E}^{j}\right)\right)$. There is an upper bound Verts $\left(b_{0}\right)$ to the number of vertex groups of the principal cyclic JSJ of a limit group with first betti number $b_{0}$. If $\|\mathcal{G}\|>\operatorname{Verts}\left(b_{0}\right) \cdot \operatorname{NInj}\left(b_{0}, d_{0}-1, e_{0}+2 b_{0}\right)$ there is some index $l$ such that $\mathcal{H}(l) \rightarrow \mathcal{G}(l)$ is injective on all vertex groups. By the last bullet of Lemma 9.21, $\mathcal{H}(l) \rightarrow \mathcal{G}(l)$ is an isomorphism.

\subsection{Proof of Lemma 9.21}

9.3.1 Graphs of spaces and immersions In this subsection we are given a fixed adjunction $G \hookrightarrow H \rightarrow G^{\prime}$ of limit groups. By a graph of spaces representing $a$ principal cyclic decomposition of a limit group $G$ we mean a graph of spaces of the following form:

- For each rigid vertex group $R$ a space $X_{R}$. Let $\mathcal{E}(R)$ be the edge groups incident to $R$, and for each $E \in \mathcal{E}(R)$ let $\sqrt{E}$ be the maximal cyclic subgroup of $R$ containing the image of $E$. For each $E \in \mathcal{E}$ there is an embedded copy $S_{E}$ of $S^{1}$ in $X_{R}$ representing the conjugacy class of $\sqrt{E}$.

- For each edge $E$, a copy $T_{E}$ of $S^{1}$, with basepoint $b_{E}$ and an edge space $T_{E} \times \mathrm{I}$. On occasion we confuse $T_{E}$ with $T_{E} \times \frac{1}{2}$, and sometimes refer to $T_{E}$ as the edge space. The interval $b_{E} \times I$ is denoted $t_{E}$, and choose a fixed but arbitrary orientation for $t_{E}$. The end of the edge space associated to $E$ is attached via the (finite sheeted!) covering map $T_{E} \rightarrow S_{E}$ representing $E \hookrightarrow \sqrt{E}$.

- For each abelian vertex group a torus $T_{A}$. If $A$ is infinite cyclic then $T_{A}$ has a basepoint $b_{A}$ and the incident edge maps are simply covering maps which send $b_{E}$ to $b_{A}$. These covering maps are isomorphisms unless the edge is adjacent to a $\mathrm{QH}$ vertex group, in which case they may be proper. For each edge space edge $E$ adjacent to $A$, an edge space $T_{E} \times I$ and an embedded copy of $T_{E}$ in $T_{A}$. This assumes edge groups not adjacent to $\mathrm{QH}$ vertex groups are primitive. Though there may be $\mathrm{QH}$ vertex groups, the cases which this definition is designed to handle do not, and we let this inconsistency slide. Unlike the rigid case, the embedded $T_{E}$ need not be disjoint, though if they meet, they must coincide. We require that any two embedded $T_{E}$, differ by an element of $T_{A}$, treated now as a group.

- For each QH vertex group $Q$ a surface with boundary $\Sigma_{Q}$. 
- If an edge group $E$ is incident to a $\mathrm{QH}$ vertex group $Q$ then $T_{E}$ is identified with a boundary component of $\Sigma_{Q}$.

- The resulting graph of spaces has fundamental group $G$.

Let $\eta: G \hookrightarrow H$ be an inclusion of limit groups, and let $\Pi_{G}$ and $\Pi_{H}$ be principal cyclic decompositions of $G$ and $H$, respectively, such that $\eta$ maps vertex groups to vertex groups, edge groups to edge groups, and respects edge data, ie, if $E \hookrightarrow V$, $\eta_{\#}(E) \hookrightarrow \eta_{\#}(V)$, then the obvious square commutes. If this is the case then $\eta$ respects $\Pi_{G}$ and $\Pi_{H}$. Let $X_{H}$ be a graph of spaces representing $\Pi_{H}$. Then there is a principal cyclic decomposition $\Pi_{G}$ of $G$, a space $X_{G}$ representing $\Pi_{G}$, and an immersion $\psi: X_{G} \rightarrow X_{H}$, inducing $\eta$, of the following form:

- For each abelian vertex group $A$ there is a finite sheeted covering map $T_{A} \rightarrow \rightarrow$ $T_{\eta_{\#}(A)}$, denoted $\left.\psi\right|_{T_{A}}$. The inclusions of incident edge spaces are respected by $\eta$ :

$$
\left.\psi\right|_{\operatorname{Im}\left(T_{E}\right)}=\left.\left(T_{\eta_{\#}(E)} \hookrightarrow T_{\eta_{\#}(A)}\right) \circ \psi\right|_{T_{E}}
$$

- For each $E$ there is a finite sheeted product-respecting covering map $T_{E} \times \mathrm{I} \uparrow \rightarrow$ $T_{\eta_{\#}(E)} \times \mathrm{I}$ which maps $t_{E}$ to $t_{\eta_{\#}(E)}$. If $E$ is adjacent to a QH vertex group then the degree of the covering map is one.

- For each $R$ there is a map $X_{R} \rightarrow X_{\eta_{\#}(R)}$ such that for each edge group $E$ incident to $R$ the following diagram commutes:

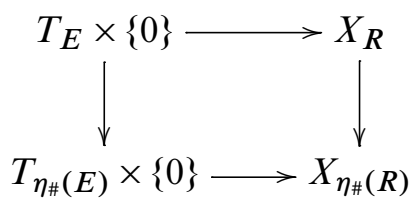

Likewise for $\times\{1\}$.

- For each QH vertex $Q$ a homeomorphism $\Sigma_{Q} \rightarrow \Sigma_{\eta_{\#}(Q)}$. The maps $X_{R} \rightarrow$ $X_{\eta_{\#}(R)}$ (similarly for $T_{A}$ 's) respect attaching maps of boundary components of surfaces.

- If $E_{1}$ and $E_{2}$ are incident to $A$ and $T_{E_{1}}$ and $T_{E_{2}}$ have the same image in $T_{A}$, then $\eta_{\#}\left(E_{1}\right) \neq \eta_{\#}\left(E_{2}\right)$.

The existence of immersions as above is an easy variation on Stallings's folding. One way to construct immersions of graphs representing subgroups is to pass to the cover of a graph representing a subgroup and trimming trees. See Stallings [15; 16]. There is also an analogous construction in this context. 


\subsubsection{Roots, immersions and resolving}

Definition 9.23 (Edge path) Let $X_{G}$ be a graph of spaces representing a principal cyclic decomposition of $G$. The zero skeleton of $X_{G}$, denoted $X_{G}^{0}$, is the disjoint union of vertex spaces.

An edge path in a graph of spaces $X_{G}$ is a map $p:[0,1] \rightarrow X_{G}$ such that $p^{-1}\left(X_{G}^{0}\right)$ contains $\{0,1\}$ and is a collection of closed subintervals. Let $[a, b]$ be the closure of a complementary component of $p^{-1}\left(X_{G}^{0}\right)$. Then we require that $p$ map $[a, b]$ homeomorphically to some $t_{E}$.

Let $X_{R}$ be a vertex space. Let $\partial X_{R}$ be the disjoint union of copies of edge spaces contained in $X_{R}$. An edge path $p$ is reduced if every restriction $\left.p\right|_{[a, b]}([a, b],\{a, b\}) \rightarrow$ $\left(X_{R}, \partial X_{R}\right)$ does not represent the trivial element in the relative homotopy group $\pi_{1}\left(X_{R}, \partial X_{R}\right)$

A continuous map $\gamma: \mathrm{S}^{1} \rightarrow X_{G}$ is cyclically reduced if all edge-path restrictions of $\gamma$ to subintervals $\mathrm{I} \subset \mathrm{S}^{1}$ are reduced edge paths.

Lemma 9.24 If $g \in G$ then there is a cyclically reduced edge path $\gamma: \mathrm{S}^{1} \rightarrow X_{G}$ representing the conjugacy class $[g]$.

Let $\psi: X_{G} \rightarrow X_{H}$ be an immersion representing $G \hookrightarrow H$. If $\gamma: \mathrm{S}^{1} \rightarrow X_{G}$ is a reduced edge path then $\psi \circ \gamma$ is a reduced edge path in $X_{H}$.

This is standard and follows easily from Stallings' folding and the definitions.

For each edge $E$ of $X_{G}$, we introduced a subset $t_{E}$ of the edge space $T_{E} \times I$. We think of $t_{E}$ as a formal element representing the path $I \rightarrow b_{E} \times I$ with some fixed but arbitrary orientation. Let $\tau\left(t_{E}\right)$ be the image of the basepoint of $T_{E}$ in the vertex space of $X_{G}$ at the terminal end of $T_{E} \times \mathrm{I}$, and let $\iota\left(t_{E}\right)$ be the image of the basepoint in the vertex space at the initial end of $T_{E}$. Then every nonelliptic element represented by a cyclically reduced path can be thought of as a composition $t_{E}$ 's, their inverses, and elements of relative homotopy groups of vertex spaces. Moreover, if the subword ${ }^{t}{ }_{E} g t_{E}^{-1}$ appears then $g$ is not contained in the image of $E$, otherwise $\gamma$ could be shortened.

Let $\gamma \in G$ be represented by a cyclically reduced edge path $\gamma ; \psi \circ \gamma$ is an edge path in $X_{H}$, and if it is not cyclically reduced, then for some subpath $t_{E} h t_{E}^{-1}$ of $\gamma$ (we may need to reverse the orientation of $t_{E}$ ), the image of this subpath is homotopic into $\eta_{\#}\left(T_{E}\right)$, which means that $[h] \in \eta_{\#}(E)$. Since $\gamma$ is reduced, $[h] \notin E$, and since the image of $E$ in $\eta_{\#}(E)$ is finite index, for some $l>0,[h]^{l} \in E$. Since edge groups 
are primitive unless adjacent to $\mathrm{QH}$ vertex groups, $E$ must be attached to a boundary component of a $\mathrm{QH}$ vertex. This implies that $\eta_{\#}(E)$ is also attached to a boundary component of a $\mathrm{QH}$ vertex group, but by standing assumption $E \rightarrow \eta_{\#}(E)$ is an isomorphism, contradicting the fact that $[h] \notin E$.

Let $G$ and $H$ be freely indecomposable limit groups, $H$ obtained from $G$ by adjoining roots to $\mathcal{E}, \eta$ : $G \hookrightarrow H$. Let $\Pi_{G}$ and $\Pi_{H}$ be principal cyclic decompositions and suppose that $K$ is elliptic in $\Pi_{G}$ if and only if $\eta(K)$ is elliptic in $\Pi_{H}$. Let $\psi: X_{G} \rightarrow X_{H}$ be an immersion representing the inclusion.

Without loss of generality, suppose that all elements of $\mathcal{E}$ are self-centralized and nonconjugate. Let $\mathcal{E}_{e}$ be the elements of $\mathcal{E}$ which are elliptic in $\Pi_{G}$ and let $\mathcal{E}_{h}$ be the elements of $\mathcal{E}$ which are hyperbolic in $\Pi_{G}$.

For each $E \in \mathcal{E}$ let $T_{E}$ be a torus representing $E, T_{F(E)}$ a torus representing $F(E)$, and let $T_{E} \rightarrow T_{F(E)}$ be the covering map corresponding to the inclusion $E \hookrightarrow F(E)$. Let $M_{E}$ be the mapping cylinder of the covering map. If $\langle\gamma\rangle \in \mathcal{E}$ we abuse notation and refer to $M_{\langle\gamma\rangle}$ as $M_{\gamma}$. The copy of $T_{F(E)}$ in $M_{E}$ is the core of $M_{E}$, and if $E$ is infinite cyclic, it is the core curve. The copy of $T_{E}$ in $M_{E}$ is the boundary, and is denoted $\partial M_{E}$.

For each element $E \in \mathcal{E}_{e}$, let $f_{E}: T_{E} \rightarrow X_{G}$ be a map representing the inclusion $E \hookrightarrow G$ which has image in a vertex space of $X_{G}$. If $E$ is an abelian vertex group of $\Pi_{G}$ then we identify $T_{E}$ with the torus $T_{A} \subset X_{G}$. For each $\langle\gamma\rangle \in \mathcal{E}_{h},{ }^{5}$ represent $\gamma$ by a reduced edge path, abusing notation, $\gamma: \partial M_{\gamma} \rightarrow X_{G}$.

Build a space $X_{G}^{\prime}$ by attaching the $M_{E}$ and $M_{\gamma}$ to $X_{G}$ along $T_{E}$ and $\operatorname{Im}(\gamma)$ by the maps $f_{E}$ and $\gamma$, respectively.

By hypothesis there is a $\pi_{1}$-surjective map $\psi^{\prime}: X_{G}^{\prime} \rightarrow X_{H}$ extending $X_{G} \rightarrow X_{H}$. We choose this map carefully: For $E \in \mathcal{E}_{e}, F(E)$ has elliptic image in $\Pi_{H}$. Choose a map $T_{F(E)} \rightarrow X_{H}$ with image contained in the appropriate vertex space of $X_{H}$, and extend the map across $M_{E}$ so that $M_{E}$ also has image contained in the vertex space of $X_{H}$. For $\langle\gamma\rangle \in \mathcal{E}_{h}$, the core curve of $M_{\gamma}$ is a $k_{\gamma}$-th root of $\gamma$. Choose a cyclically reduced representative of $\sqrt[k \gamma]{\gamma}: S^{1} \rightarrow X_{H}$ and let the map on the core curve agree with this representative.

The restriction of $\psi^{\prime}$, defined thus far, to the disjoint union of $X_{G}$ and the core curves of the $M_{\gamma}$, is transverse to the subsets $T_{\eta_{\#}(E)} \times\left\{\frac{1}{2}\right\}$. Extend $\psi^{\prime}$ to $X_{G}^{\prime}$ so the composition

$$
M_{\gamma} \hookrightarrow X_{G}^{\prime} \stackrel{\psi^{\prime}}{\rightarrow} X_{H}
$$

${ }^{5}$ All elements of $\mathcal{E}_{h}$ are infinite cyclic. 
is transverse to all $T_{\eta_{\#}(E)} \times \frac{1}{2}$. Let $\Lambda$ be the preimage

$$
\psi^{\prime-1}\left(\bigsqcup_{E \in \mathcal{E}(G)}\left(T_{\eta_{\#}(E)} \times\left\{\frac{1}{2}\right\}\right)\right) .
$$

Suppose some component of $\Lambda$ is a circle which misses the boundary and core of some $M_{\gamma}$. By transversality this component of $\Lambda$ is a one manifold without boundary, and is therefore a circle. If this circle bounds a disk then there is a map homotopic $\psi^{\prime}$, which agrees with $\psi^{\prime}$ on the core curves and $X_{G}$ such that the number of connected components of the preimage is strictly lower. If the circle doesn't bound a disk in $M_{\gamma}$ then it is boundary parallel. If this is the case then $\gamma$ is elliptic and we have a contradiction.

Fix a mapping cylinder $M_{\gamma}$ and consider the preimage of $\Lambda$ under the map $M_{\gamma} \rightarrow X_{G}^{\prime}$. The preimage is a graph all of whose vertices are contained in the core curve of $M_{\gamma}$ or in the boundary of $M_{\gamma}$. If any component of the preimage of $\Lambda$ doesn't connect the boundary of $M_{\gamma}$ to the core curve, then it is an arc and there is an innermost such arc which can be used to show that one of either $\gamma$ or $\gamma^{\prime}$ was not reduced. Thus the preimages of arcs connect the core curve to the boundary.

Let $b$ be a point of intersection of $\Lambda$ with the core curve of $M_{\gamma}$. There are $k_{\gamma}$ arcs, where $k_{\gamma}$ is the degree of the root added to $\gamma, s_{1}, \ldots, s_{k_{\gamma}}$ (cyclically ordered by traversing $\partial M_{\gamma}$ ) in $\Lambda$ connecting $b$ to $\partial M_{\gamma}$. Now consider the arcs as paths $s_{j}:[0,1] \rightarrow M_{\gamma}$. The composition $p_{\gamma}:=s_{2}^{-1} s_{1}$ is a path in $M_{\gamma}$ from $\partial M_{\gamma}$ to $\partial M_{\gamma}$. Let $D_{\gamma}$ be the subpath of $\gamma$ obtained by traversing $\partial M_{\gamma}$ from $*:=s_{1} \cap \partial M_{\gamma}$ to $*_{2}:=s_{2} \cap \partial M_{\gamma}$. The path $D_{\gamma} p_{\gamma}$ is homotopic, relative to $*$, to $s_{1}^{-1} \sqrt[k \gamma]{\gamma} s_{1}$. In particular,

$$
\left(D_{\gamma} p_{\gamma}\right)^{k_{\gamma}} \simeq \gamma
$$

A typical neighborhood of a component of $\Lambda$ is illustrated in Figure 6.

Sublemma 9.25 Suppose $\eta$ : $G \hookrightarrow H$ is indecomposable, $H$ obtained from $G$ by adjoining roots to $\mathcal{E}$. Let $\Pi_{H}$ be a one-edged splitting of $G$ over a cyclic edge group $E_{H}$. Let $\Pi_{G}$ be the splitting $G$ inherits from its action via $\eta$ on the BassSerre tree for $\Pi_{H}$. Represent $\Pi_{H}$ by a graph of spaces $X_{H}$, and choose a graph of spaces $X_{G}$ and an immersion $\psi: X_{G} \rightarrow X_{H}$ representing $\eta$. Suppose that $\Pi_{G}$ is one-edged, and that the edge group is $E$. If $\mathcal{E}_{h}$ is nonempty then $E \hookrightarrow \eta_{\#}(E)$ is a proper finite index inclusion.

Proof Let $\langle\gamma\rangle \in \mathcal{E}_{h}$, and represent $\gamma$ by a reduced edge path crossing $t_{E}$. Since $\psi$ is one-to-one on edge spaces, $p_{\gamma}$ is a closed path. As such, it represents an element 


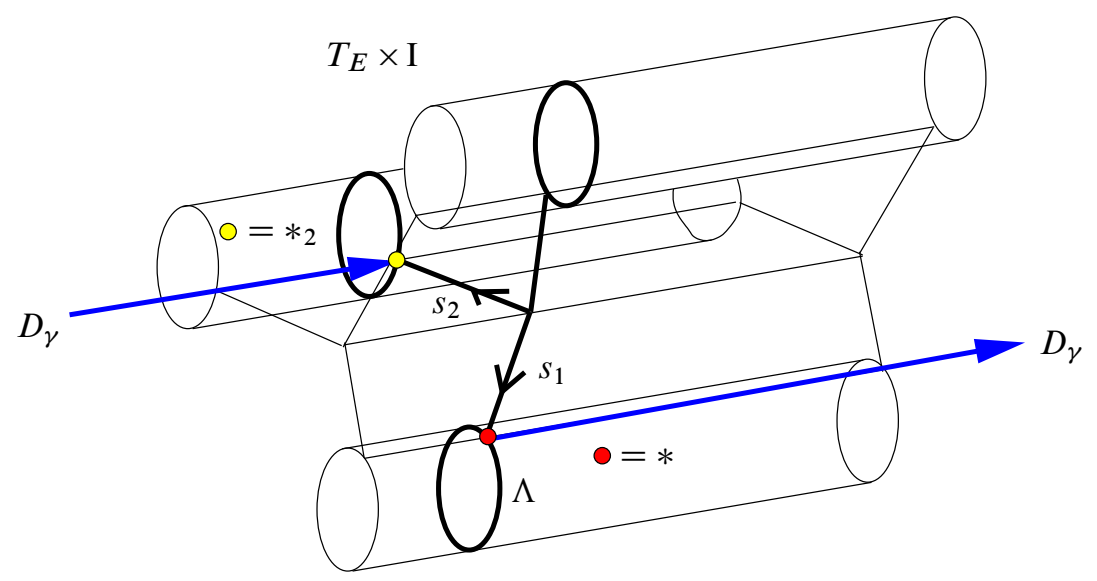

Figure 6: A neighborhood of a component of $\Lambda$ in $X_{G}^{\prime}$

of the fundamental group of $X_{G}^{\prime}$. Then $\left[\psi \circ p_{\gamma}\right] \in \eta_{\#}(E)$. If $\left[\psi \circ p_{\gamma}\right] \in \operatorname{Im}(E)$ then there is a path $p_{\gamma}^{\prime}$ in $T_{E}$ which is homotopic in $T_{\eta_{\#}(E)}$, relative to the image of $*$, to $\psi \circ p_{\gamma}$. Let $\alpha=D_{\gamma} p_{\gamma}^{\prime}$. Then $\psi \circ \alpha$ is homotopic rel the image of $*$ to $\psi \circ D_{\gamma} p_{\gamma}$. But then $[\alpha]^{k_{\gamma}}=\gamma$ contradicting indivisibility of $\gamma$.

Sublemma 9.26 Let $G \hookrightarrow G^{\prime}$ be an adjunction of roots. Let $\Pi_{G^{\prime}}$ be a principal cyclic splitting of $G^{\prime}$ with one abelian vertex group $A^{\prime}$, let $\Pi_{G}$ be the associated splitting of $G$, and represent $G \hookrightarrow G^{\prime}$ by an immersion $\eta: X_{G} \hookrightarrow X_{G^{\prime}}$ reflecting $\Pi_{G}$ and $\Pi_{G^{\prime}}$. Suppose there is a unique vertex group $A^{\prime}$ of $\Pi_{G}, A^{\prime} \in \mathcal{E}$, mapping to $A$, and that no other element of $\mathcal{E}$ is conjugate into $A^{\prime}$. If $\eta$ is one-to-one on edges adjacent to $A^{\prime}$ then the induced map $F\left(A^{\prime}\right) \rightarrow A / P(A)$ is onto.

Proof Let $E_{1}, \ldots, E_{n}$ be the edges adjacent to $A^{\prime}$, and set $F\left(E_{i}\right)=\eta_{\#}\left(E_{i}\right)=P(A)$. Let $H$ be the limit group defined as follows: Let $\Delta=\Delta\left(R_{j}, E_{i}, A^{\prime}\right)$ be a graphs of groups representation of $\Pi_{G}$. Let $\mathcal{E}_{R_{j}}$ be the subcollection of $\mathcal{E}$ consisting of elements conjugate into $R_{j}$. Let

$$
\begin{aligned}
& S_{j}:=\operatorname{Im}_{G^{\prime}}\left(\left\langle R_{j}\left[\sqrt{\mathcal{E}_{R_{j}}}\right], g F\left(E_{i}\right) g^{-1}\right\rangle_{g E_{i} g^{-} 1<R_{j}}\right), \\
& A^{\prime \prime}:=\operatorname{Im}_{G^{\prime}}\left(F\left(A^{\prime}\right), P(A)\right)
\end{aligned}
$$

Let $H:=\Delta\left(S_{j}, F\left(E_{i}\right), A^{\prime \prime}\right)$ There are maps $G \hookrightarrow H \hookrightarrow G^{\prime}$. To show that $H \hookrightarrow G^{\prime}$ is actually surjective we need to find, for each $\langle\gamma\rangle \in \mathcal{E}_{h}$, a $k_{\gamma}$-th root in $H$. But this is the argument given in Sublemma 9.25. Let $G^{\prime} \rightarrow A / P(A)$ be the map which kills all vertex, edge groups, and stable letters, other than $A$. The quotient map kills everything except $A$ and $F\left(A^{\prime}\right)$, giving the desired surjection. 
Sublemma 9.27 Let $G \hookrightarrow H \rightarrow G^{\prime}$ be an adjunction without any proper extensions. Suppose $G$ is freely indecomposable, has no $\mathrm{QH}$ vertex groups, and $\operatorname{JComp}(G)=$ $\operatorname{JComp}\left(G^{\prime}\right)$. Represent the $G \hookrightarrow H$ by an immersion $X_{G} \rightarrow X_{H}$, representing $\operatorname{JSJ}_{H}(G)$, and $\operatorname{RJSJ}(H)$, respectively. Then $v$ is one-to-one on edge spaces.

Furthermore, $\operatorname{JSJ}_{H}(G)=\operatorname{PJSJ}\left(G, \mathcal{E}_{H}^{\text {ell }}\right)$ and $\operatorname{JSJ}_{H}^{*}\left(G^{\prime}\right)=\operatorname{PJSJ}\left(G^{\prime}, \eta_{\#}\left(\mathcal{E}_{H}^{\text {ell }}\right)\right)$. Every vertex group $W$ of $H$ is obtained from a vertex group $V$ of $\operatorname{JSJ}_{H}(G)$ by adjoining roots to the elements of $\mathcal{E}$ which are conjugate into $V$ and edge groups incident to $V$.

Proof Represent $G \hookrightarrow H$ by an immersion $X_{G} \rightarrow X_{H}$, where $X_{G}$ represents $\operatorname{PJSJ}\left(G, \mathcal{E}_{H}^{\text {ell }}\right)$ and $X_{H}$ represents the principal cyclic JSJ of $H$. For each edge $E_{i}$ of $X_{G}$ let $e_{i}$ be a generator, let $k_{i}$ be the largest degree of a root of $e_{i}$ in $H$, let $F(E)=\left\langle f_{i}\right\rangle$, and let $E \hookrightarrow F(E)$ be the map which sends $e_{i}$ to $f_{i}^{k_{i}}$. Let $\mathcal{E}^{\prime}$ be the collection of elements of $\mathcal{E}$ which are elliptic in $H$ along with all edge groups of $\operatorname{JSJ}_{H}(G)$.

Consider the group $G\left[\sqrt{\mathcal{E}^{\prime}}\right]$. Let $\Delta=\Delta\left(R_{i}, A_{j}, E_{k}\right)$ be a graph of groups representation of $\operatorname{PJSJ}\left(G, \mathcal{E}_{H}^{\text {ell }}\right)$. Let $\mathcal{E}_{R_{i}}$ be the set of elements of $\mathcal{E}^{\prime}$ which are conjugate into $R_{i}$. Likewise, let $\mathcal{E}_{A_{j}}$ be the set of elements of $\mathcal{E}^{\prime}$ which are conjugate into $A_{j}$. Now build $H^{\prime}=\boldsymbol{S}\left(G\left[\sqrt{\mathcal{E}^{\prime}}\right]\right) \rightarrow H$. Choose a graph of spaces $X_{H^{\prime}}$ representing $\boldsymbol{S}(\Delta)$.

There is a pair of maps of graphs of spaces $X_{G} \rightarrow X_{H^{\prime}}, X_{H^{\prime}} \rightarrow X_{H}$, and there is an epimorphism $G\left[\sqrt{\mathcal{E}^{\prime}}\right] \rightarrow H^{\prime}$. The map $\psi^{\prime}: X_{G} \rightarrow X_{H^{\prime}}$ is one-to-one on edge spaces. Moreover, $H^{\prime}$ is a limit group since the map $H^{\prime} \rightarrow H$ is strict.

The proof of the lemma is almost complete if $\psi^{\prime}$ extends to $X_{G}^{\prime}$, that is, if $H^{\prime}$ contains all roots of elements adjoined to $\mathcal{E}$. Then the image of $G[\sqrt{\mathcal{E}}]$ (with the induced graph of groups decomposition) in $H^{\prime}$ is a nontrivial extension of $H$.

Consider the paths $D_{\gamma}$ and $p_{\gamma}$ defined previously through resolving. We defined $p_{\gamma}:=s_{2}^{-1} s_{1}$ and set $*=s_{1} \cap \partial M_{\gamma}$. Let $*_{2}:=s_{2} \cap \partial M_{\gamma}$. To show that $H^{\prime}$ has a $k_{\gamma}$-th root of $\gamma$ we need to show that $X_{H^{\prime}}$ has a path $p_{\gamma}^{\prime}$ from the image of $*_{2}$ to the image of $*$ whose image under $X_{H^{\prime}} \rightarrow X_{G^{\prime}}$ is homotopic rel endpoints to the image of $p_{\gamma}$.

Suppose that $T_{E_{1}} \times \frac{1}{2}$ and $T_{E_{2}} \times \frac{1}{2}$ are the midpoints of edge spaces containing $*$ and $*_{2}$, respectively, and suppose, without loss of generality, that $D_{\gamma}$ starts and ends by traversing the second and first halves of $T_{E_{1}}$ and $T_{E_{2}}$, respectively, in the positive direction. Choose the orientations of $t_{E_{i}}$ so that the terminal endpoints of $t_{E_{1}}$ and $t_{E_{2}}$ are both contained in some $T_{A}: E_{1}$ and $E_{2}$ are conjugate in $H$, must therefore be conjugate in $G$ since $\operatorname{JComp}\left(G^{\prime}\right)=\operatorname{JComp}(G)$, and cannot both be adjacent to a rigid vertex group of $G$, otherwise there is a rigid vertex group $R$ of $G$ such that 
$v(R)>v\left(\eta_{\#}(R)\right)$. The only other possibility is that they are both adjacent to an abelian vertex group $A$, as claimed.

Let $t_{\varphi_{\#}}^{+}\left(E_{i}\right)$ be the half of $t_{\varphi_{\#}\left(E_{i}\right)}$ obtained by traversing $t_{\varphi_{\#}}\left(E_{i}\right)$ from the midpoint to the terminal endpoint. By Sublemma 9.26, $H^{\prime} \rightarrow H$ is surjective on abelian vertex groups, and, by construction, the terminal endpoints of $t_{\varphi_{\#}}^{+}\left(E_{i}\right)$ agree. Let $p_{\gamma}^{\prime \prime}:=t_{\varphi_{\#}\left(E_{2}\right)}^{+}\left(t_{\varphi_{\#}\left(E_{1}\right)}^{+}\right)^{-1}$. Then $p_{\gamma}^{\prime \prime}$ is a path from $\varphi\left(*_{2}\right)$ to $\varphi(*)$ whose image in $X_{H}$ is homotopic rel endpoints into $\psi_{\#}\left(E_{1}\right)\left(=\psi_{\#}\left(E_{2}\right)\right)$. Since $H^{\prime} \rightarrow H$ is surjective on edge groups, there is a closed path $h_{\gamma}$ in $\left(T_{\varphi_{\#}}\left(E_{1}\right), \varphi(*)\right)$ which maps to the image of $p_{\gamma}$. Set $p_{\gamma}^{\prime}:=h_{\gamma} p_{\gamma}^{\prime \prime}$. The image of $p_{\gamma}^{\prime}$ is homotopic rel endpoints to the image of $p_{\gamma}$ in $X_{H}$. Arguing as in Sublemma 9.25, $\left(\varphi \circ D_{\gamma}\right) p_{\gamma}^{\prime}$ is a $k_{\gamma}$-th root of $\varphi \circ \gamma$ and the map $X_{G}^{\prime} \rightarrow X_{H}$ factors through $X_{H^{\prime}}$.

Thus there is a map $X_{G}^{\prime} \rightarrow X_{H^{\prime}}$ extending $X_{G} \rightarrow X_{H}$. Since $H$ has no proper extensions, $\operatorname{Im}_{H^{\prime}}(G[\sqrt{\mathcal{E}}]) \rightarrow H$ is an isomorphism. In particular, $X_{G} \rightarrow X_{H}$ is oneto-one on edges and $E_{1}=E_{2}$. Now that $H^{\prime} \cong H, S(\Delta)$ must be the principal cyclic JSJ of $H$. If there is a principal cyclic splitting of $H$ not visible in $\Delta$ then it must be a cyclic splitting inherited from the relative (to incident edge groups) principal cyclic JSJ decomposition (the JSJ decomposition associated to the collection of all principal cyclic splittings in which some collection of subgroups is elliptic) of some vertex group of $\Delta$. On the other hand, all vertex groups of $\Delta$ must be elliptic in the principal cyclic JSJ of $H$ since they are obtained by adjoining roots to subgroups of $G$ which are guaranteed to be elliptic in the principal cyclic JSJ of $H$.

This nearly completes the proof of Lemma 9.21. We need to prove that the vertex groups of $\operatorname{JSJ}_{H}^{*}\left(G^{\prime}\right)$ are obtained from the images of the vertex groups of $\operatorname{JSJ}(H)$ by adjoining roots, and that $\pi$ is injective if its restrictions to vertex groups are injective.

Let $\Delta=\Delta\left(R_{i}, A_{j}, E_{k}\right)$ be a graph of groups decomposition representing the principal cyclic JSJ of $H$. We know that all vertex and edge groups of $\Delta$ map to vertex and edge groups of $\mathrm{JSJ}_{H}^{*}\left(G^{\prime}\right)$. Let $\boldsymbol{S}(\pi): S(H) \rightarrow G^{\prime}$ be the strict homomorphism constructed in Section 5, and let $\boldsymbol{S}(\Delta)$ be the principal cyclic decomposition of $\boldsymbol{S}(H)$ in which all images of vertex groups of $\Delta$ are elliptic. Strict $S(\pi)$ maps elliptic subgroups of $\boldsymbol{S}(\Delta)$ to elliptic subgroups of $\mathrm{JSJ}_{H}^{*}\left(G^{\prime}\right)$. Moreover, if $A$ is a noncyclic abelian vertex group of $H$, then $S(\pi)$ maps $A / P(A)$ onto $\pi_{\#}(A) / P\left(\pi_{\#}(A)\right)$. Thus all modular automorphisms of $\boldsymbol{S}(H)$ supported on abelian vertex groups of $\Delta$ push forward to modular automorphisms of $G^{\prime}$. Since $S(H) \rightarrow G^{\prime}$ is clearly one to one on the set of edge groups adjacent to every vertex group, every Dehn twist of $\boldsymbol{S}(H)$ pushes forward to a Dehn twist of $G^{\prime}$. If $\pi$ is injective on vertex groups, then by Lemma $2.14 S(\pi)$ is an isomorphism. The fourth bullet follows immediately from the definition of $\boldsymbol{S}$ and the fact that all edge groups in the directed sequence are infinite cyclic. 


\section{Lifting dimension bounds}

Given a chain $\mathcal{L}$ there is the identity resolution $\mathcal{L} \square_{\text {id }} \mathcal{L}$ of complexity at most $(\operatorname{rk}(\mathcal{L}), 3 \operatorname{rk}(\mathcal{L}))$.

Corollary 10.1 (Reduction to indecomposable sequences) Let $\iota: \mathcal{G} \hookrightarrow \mathcal{L}$ be an inclusion of sequences. For all $K$ there exists $M=M(K, \operatorname{Comp}(\iota))$ such that if $\|\mathcal{G}\|>M$ there exists a maximal resolution $\widetilde{\mathcal{G}} \square \mathcal{G} \hookrightarrow \mathcal{L}$ of $\mathcal{G}$ such that $\|\widetilde{\mathcal{G}}\|>K$ such that $c_{\mathrm{fd}}$ is constant along $\widetilde{\mathcal{G}}$.

In particular, $\widetilde{\mathcal{G}}$ splits as a graded free product of sequences

$$
\widetilde{\mathcal{G}}=\widetilde{\mathcal{G}}_{1} * \cdots * \widetilde{\mathcal{G}}_{p} * \mathcal{F}
$$

where $\mathcal{F}$ is the constant sequence $\left(\mathbb{F}_{q}\right)$ for some $q$. The sequences $\widetilde{\mathcal{G}}_{i} \square \mathcal{G}$ are indecomposable maximal resolutions of their images, and we may assume that they are QPJSJ-respecting. If $\operatorname{sc}(\widetilde{\mathcal{G}})>(1,0)$ then

$$
\operatorname{Comp}\left(\widetilde{\mathcal{G}}_{i} \square \mathcal{L}\right)<\operatorname{Comp}(\mathcal{G} \hookrightarrow \mathcal{L}) .
$$

Proof of Corollary 10.1 By Lemma 2.28, the rank of $\mathcal{G}$ is bounded above by some function of $\operatorname{Comp}(\mathcal{G})$. Now use Theorem 5.5. To guarantee that each $\widetilde{\mathcal{G}}_{i}$ is QPJSJ-respecting observe that there are at most $b_{1}(\mathcal{G})$ free factors. Now argue as in Corollary 7.7.

Theorem 10.2 Let $\left(b_{0}, d_{0}\right)$ be a minimal complexity for which Theorem 3.4 fails, should there be one, and suppose $\mathcal{G} \hookrightarrow \mathcal{L}$ satisfies the hypothesis of Corollary 10.1. Let $\widetilde{\mathcal{G}} \square \mathcal{L}$ be the resolution provided by the corollary. Then $\widetilde{\mathcal{G}}$ is indecomposable, $c_{a}(\widetilde{\mathcal{G}})=0$, and $\widetilde{\mathcal{G}}$ has no $\mathrm{QH}$ subgroups.

Proof If $\widetilde{\mathcal{G}}$ is freely decomposable it is a nontrivial free product of resolutions

$$
\left(\widetilde{\mathcal{G}}_{1} * \cdots * \widetilde{\mathcal{G}}_{p} * \mathcal{F}_{q}\right) \square \mathcal{L}
$$

and each free factor is a maximal resolution

$$
\widetilde{\mathcal{G}}_{i} \square \mathcal{L}, \quad \mathcal{F}_{q} \square \mathcal{L}
$$

of complexity strictly less than $\operatorname{Comp}(\mathcal{G} \square \mathcal{L})$, with equality only if $(p, q)=(1,0)$.

Suppose $p=1, q>0$ or $p \geq 2$. In this case there are at most $b_{1}(\mathcal{G})$ free factors of $\widetilde{\mathcal{G}}$, each of which has complexity strictly less than that of $\mathcal{L}$. By induction there is a constant $B$ which bounds the proper lengths of resolutions with complexity less than 
$\operatorname{Comp}(\mathcal{G} \square \mathcal{L})$. If $M>3 B \mathrm{~b}_{1}(\mathcal{G})$ there exist three consecutive indices $i, i+1, i+2$ such that the maps

$$
\widetilde{\mathcal{G}}_{j}(i) \rightarrow \widetilde{\mathcal{G}}_{j}(i+1) \rightarrow \widetilde{\mathcal{G}}_{j}(i+2)
$$

are isomorphisms for all $j$. The same is automatically true for the free part of $\tilde{\mathcal{G}}$. It follows immediately that $\mathcal{G}\left(k_{i}\right) \rightarrow \mathcal{G}\left(k_{i+1}\right)$ is an isomorphism.

We must be in the case $(p, q)=(1,0)$. As before, if $\mathcal{L}$ has length at least $M=$ $M(K, \operatorname{Comp}(\mathcal{G} \hookrightarrow \mathcal{L}))$, and $\mathcal{G} \hookrightarrow \mathcal{L}$ satisfies Corollary 7.7, the sequence $\widetilde{\mathcal{G}}$ has length at least $K$, is indecomposable, QPJSJ-respecting, and the sequence $c_{a}(\widetilde{\mathcal{G}})$ is constant. We now show how to lift a dimension bound for sequences simpler than $\mathcal{G}$ if $c_{a}(\widetilde{\mathcal{G}})>0$.

Since $c_{a}$ is constant, as is the number of abelian vertex groups, along $\widetilde{\mathcal{G}}$, by construction, the maps $\widetilde{\mathcal{G}}(i) \rightarrow \widetilde{\mathcal{G}}(j)$ map $\widetilde{\mathcal{G}}(i)_{P}$ onto $\widetilde{\mathcal{G}}(j)_{P}$. If $\widetilde{\mathcal{G}}(i) \rightarrow \widetilde{\mathcal{G}}(j)$ is JSJrespecting, every automorphism in $\operatorname{Mod}\left(\widetilde{\mathcal{G}}(i), \widetilde{\mathcal{G}}(i)_{P}\right)$ pushes forward to an element of $\operatorname{Mod}\left(\widetilde{\mathcal{G}}(j), \widetilde{\mathcal{G}}(j)_{P}\right)$, hence if the map $\widetilde{\mathcal{G}}(i)_{P} \rightarrow \widetilde{\mathcal{G}}(j)_{P}$ is an isomorphism then $\widetilde{\mathcal{G}}(i) \rightarrow \widetilde{\mathcal{G}}(j)$ is $\widetilde{\mathcal{G}}(i)_{P}$-strict. By Lemma $2.14 \widetilde{\mathcal{G}}(i) \rightarrow \widetilde{\mathcal{G}}(j)$ will be an isomorphism. If $c_{a}(\widetilde{\mathcal{G}})>0$ then $\mathrm{b}_{1}\left(\widetilde{\mathcal{G}}_{P}\right)<\mathrm{b}_{1}(\widetilde{\mathcal{G}})$ and

\section{$\operatorname{Comp}\left(\widetilde{\mathcal{G}}_{P} \square \mathcal{G}\right)<\operatorname{Comp}(\widetilde{\mathcal{G}} \square \mathcal{G})$.}

Suppose $\tilde{\mathcal{G}}$ has a sequence of $\mathrm{QH}$ subgroups. Then $\tilde{\mathcal{G}}$ respects the restricted principal cyclic decompositions and maps the vertex groups of $\operatorname{RJSJ}(\widetilde{G}(i))$ onto the associated vertex groups of $\operatorname{RJSJ}(\widetilde{\mathcal{G}}(i+1))$. Thus we derive boundedly many (in $\left.\mathrm{b}_{1}(\widetilde{G})\right)$ sequences $\mathcal{V}_{i} \hookrightarrow \widetilde{\mathcal{G}}$ of vertex groups. The resolutions $\mathcal{V}_{i} \square \mathcal{L}$ have strictly lower complexity than $\widetilde{\mathcal{G}} \square \mathcal{L}$. Thus, given $K$ there exists $M\left(\mathrm{~b}_{1}(\mathcal{G}), K\right)$ such that of $\|\widetilde{\mathcal{G}}\|>M$ then it has a subsequence of length $K$ such that all maps on vertex groups are injective. As above, modular automorphisms from $\operatorname{Mod}(\mathcal{G}, \operatorname{RJSJ}(\mathcal{G}))$ push forward and some $\mathcal{G}(i) \rightarrow \mathcal{G}(i+1)$ must be an isomorphism by Lemma 2.14 . We may therefore assume that the QPJSJ-respecting resolutions have no QH subgroups.

The theorem above contains the main idea of the proof of Theorem 3.4. If a QPJSJrespecting resolution $\tilde{\mathcal{G}} \square \mathcal{G}$ has no $\mathrm{QH}$ subgroups and $\left.c_{a}(\tilde{(} \mathcal{G})\right)=0$ we must use an analysis of sequences of images of vertex groups of $\operatorname{QPJSJ}(\widetilde{\mathcal{G}})$ to conclude that if $\widetilde{\mathcal{G}}$ is sufficiently long then it contains an isomorphism. For this we use Lemma 8.7 to find sequences of strictly lower complexity.

Theorem 7.6 is used to express QPJSJ-respecting sequences as graphs of sequences of groups obtained by passing to quotients and iteratively adjoining roots. Theorem 9.4, on the other hand, is only stated for sequences obtained by adjoining roots once, and not iteratively, at each step, along a single fixed collection of elements. We first construct a collection of subsequences of subgroups to which Theorem 9.4 is applicable. 
Let $\mathcal{R}_{i}$ be a sequence of vertex groups (not a sequence of images!) of QPJSJ $(\mathcal{G})$. Let $E_{1}, \ldots, E_{m}$ be the edge groups incident to some vertex group $\mathcal{R}_{i}^{n-1}(n-1)<\mathcal{G}(n-1)$, and let $F_{1}, \ldots, F_{m}$ be the corresponding edges of $\mathcal{G}(n)$. Let $C_{j}$ be the closure of the image of $E_{j}$ in $F_{j}$, the subgroup of $F_{j}$ consisting of all elements which have powers lying in the image of $E_{j}$. Now let $\mathcal{S}_{i}(n)=\left\langle\mathcal{R}_{i}^{n-1}(n) *_{\operatorname{Im}\left(E_{j}\right)} C_{j}\right\rangle<\mathcal{R}_{i}^{n}(n)$ and $\mathcal{S}_{i}^{n}(m)=\varphi_{n, m}\left(\mathcal{S}_{i}(n)\right)$. Then $\mathcal{S}_{i}(n)$ is obtained from $\mathcal{S}_{i}^{n-1}(n)$ by adjoining roots to the collection

$$
\mathcal{E}_{n}^{i}=\left\{\mathcal{S}_{i}^{n-1}(n) \cap C_{j}\right\}_{j=1, \ldots, m} .
$$

A priori, $\mathcal{S}_{i}^{n-1}(n)$ is only obtained from $\mathcal{R}_{i}^{n-1}(n)$ by adjoining roots, but since all edges adjacent to $\mathcal{R}_{i}$ are small, they have infinite cyclic images. Note that $\left\|\mathcal{E}_{n}^{i}\right\| \leq 2 \mathrm{~b}_{1}(\mathcal{G})$.

Theorem 10.3 (Inductive step) There is a function $D^{\prime}(D$, Comp) such that if $\mathcal{L}$ is a freely indecomposable QPJSJ-respecting chain of limit groups, and, for all sequences $\mathcal{R}_{i}^{n}<\mathcal{L}$ of images of vertex groups of QPJSJ-decompositions, $\left\|\mathcal{R}_{i}^{n}\right\|_{\mathrm{pl}}<D$ then $\|\mathcal{L}\|_{\mathrm{pl}}<D^{\prime}(D, \operatorname{Comp}(\rho))$.

Proof Suppose there exist $\mathcal{L}$ of arbitrary proper length. If the sequences $\mathcal{R}_{i}^{n}$ are constant then there are no flexible edges, the quasicyclic JSJ decomposition agrees with the principal cyclic JSJ decomposition, and all peripheral subgroups of abelian vertex groups embed in $\mathcal{L}(n+1)$. By Lemma 5.22 the envelopes of all rigid vertex groups of $\mathcal{L}(n)$ embed in $\mathcal{L}(n+1)$, therefore $\mathcal{L}(n) \rightarrow \mathcal{L}(n+1)$ is strict. Every element of the modular group of $\mathcal{L}(n)$ pushes forward to $\operatorname{Mod}(\mathcal{L}(n+1))$ and $\mathcal{L}(n) \rightarrow \mathcal{L}(n+1)$ is an isomorphism by Lemma 2.14 .

For the general case, observe that $\mathcal{S}_{i}^{n}$ is a sequence of subgroups of $\mathcal{R}_{i}^{n}$ and has proper length bounded by $D$. By Lemma 2.27 the number of sequences of vertex groups of $\operatorname{QPJSJ}(\mathcal{L})$ is bounded by $\operatorname{Verts}\left(\mathrm{b}_{1}(\mathcal{L})\right)$. If $\|\mathcal{L}\|>D^{B K}$ then there is a subsequence $\mathcal{L}^{\prime}$ of length $K$ such that for each $n$ and $i$ the sequence

$$
\mathcal{S}_{i}^{n}(n+1) \rightarrow \mathcal{S}_{i}^{n}(n+2) \rightarrow \cdots
$$

is constant. Consider the following pairs of sequences, indexed by $n$ :

$$
\left(\left(\mathcal{S}_{i}^{n-1}(K)\right),\left(\mathcal{S}_{i}^{n}(n)\right), \mathcal{E}^{i}\right), \quad n<K
$$

By Theorem 9.4, for all but $N=\operatorname{NInj}\left(b_{1}(\mathcal{L}), \operatorname{depth}(\mathcal{L}), 2 \mathrm{~b}_{1}(\mathcal{L})\right)$ indices, the map

$$
\mathcal{S}_{i}^{n-1}(n-1) \rightarrow \mathcal{S}_{i}^{n-1}(K) \cong \mathcal{S}_{i}^{n-1}(n)
$$

is an isomorphism, and if $K>N B$, then there is some index $n$ such that $\mathcal{S}_{i}^{n-1}(n-1) \rightarrow$ $\mathcal{S}_{i}^{n-1}(n)$ is an isomorphism for all $i$. 
By construction, if $\mathcal{R}_{i}^{n-1}(n-1)$ has any small flexible edges, then $\mathcal{S}_{i}^{n-1}(n-1)$ intersects at least one of them in a finite index, rank two, subgroup, but since $\mathcal{S}_{i}^{n-1}(n-1) \rightarrow$ $\mathcal{S}_{i}^{n-1}(n)$ is an isomorphism, this contradicts flexibility of that edge, therefore the QPJSJ decompositions coincide with the PJSJ decompositions, and $\mathcal{R}_{i}^{n}=\mathcal{S}_{i}^{n}$. Thus if $\|\mathcal{L}\|>D^{\prime}=D^{N B^{2}}$ then $\mathcal{L}$ contains an isomorphism.

Theorem 10.2, Lemma 8.7, and Theorem 10.3 immediately imply Theorem 3.4, hence Theorem 1.1.

\section{References}

[1] G Baumslag, A remark on generalized free products, Proc. Amer. Math. Soc. 13 (1962) 53-54 MR0133357

[2] G Baumslag, A Myasnikov, V Remeslennikov, Algebraic geometry over groups, from: "Algorithmic problems in groups and semigroups (Lincoln, NE, 1998)", (J-C Birget, S Margolis, J Meakin, M Sapir, editors), Trends Math., Birkhäuser, Boston (2000) 35-50 MR1750491

[3] M Bestvina, M Feighn, Bounding the complexity of simplicial group actions on trees, Invent. Math. 103 (1991) 449-469 MR1091614

[4] M Bestvina, M Feighn, Notes on Sela's work: limit groups and Makanin-Razborov diagrams, from: "Geometric and cohomological methods in group theory", (MR Bridson, P H Kropholler, I J Leary, editors), London Math. Soc. Lecture Note Ser. 358, Cambridge Univ. Press (2009) 1-29 MR2605174

[5] C Champetier, V Guirardel, Limit groups as limits of free groups, Israel J. Math. 146 (2005) 1-75 MR2151593

[6] M J Dunwoody, Folding sequences, from: "The Epstein birthday schrift", (I Rivin, C Rourke, C Series, editors), Geom. Topol. Monogr. 1 (1998) 139-158 MR1668347

[7] M J Dunwoody, M E Sageev, JSJ-splittings for finitely presented groups over slender groups, Invent. Math. 135 (1999) 25-44 MR1664694

[8] K Fujiwara, P Papasoglu, JSJ-decompositions of finitely presented groups and complexes of groups, Geom. Funct. Anal. 16 (2006) 70-125 MR2221253

[9] O Kharlampovich, A Myasnikov, Elementary theory of free non-abelian groups, J. Algebra 302 (2006) 451-552 MR2293770

[10] L Louder, Scott complexity and adjoining roots to finitely generated groups arXiv: math/0612222

[11] E Rips, Z Sela, Cyclic splittings of finitely presented groups and the canonical JSJ decomposition, Ann. of Math. (2) 146 (1997) 53-109 MR1469317 
[12] W Rossmann, Lie groups: An introduction through linear groups, Oxford Graduate Texts in Math. 5, Oxford Univ. Press (2002) MR1889121

[13] G P Scott, Finitely generated 3-manifold groups are finitely presented, J. London Math. Soc. (2) 6 (1973) 437-440 MR0380763

[14] Z Sela, Diophantine geometry over groups I: Makanin-Razborov diagrams, Publ. Math. Inst. Hautes Études Sci. (2001) 31-105 MR1863735

[15] J R Stallings, A topological proof of Grushko's theorem on free products, Math. Z. 90 (1965) 1-8 MR0188284

[16] J R Stallings, Topology of finite graphs, Invent. Math. 71 (1983) 551-565 MR695906

[17] J R Stallings, Foldings of $G$-trees, from: "Arboreal group theory (Berkeley, CA, 1988)", (R C Alperin, editor), Math. Sci. Res. Inst. Publ. 19, Springer, New York (1991) 355-368 MR1105341

[18] G A Swarup, Delzant's variation on Scott complexity arXiv:math.GR/0401308

[19] H Wilton, Solutions to Bestvina \& Feighn's exercises on limit groups, from: "Geometric and cohomological methods in group theory", (M R Bridson, P H Kropholler, I J Leary, editors), London Math. Soc. Lecture Note Ser. 358, Cambridge Univ. Press (2009) 30-62 MR2605175

Department of Mathematics, University of Michigan

East Hall, 530 Church Street, Ann Arbor MI 48109-1043, USA

llouder@umich.edu, lars@d503.net

http://www.math.1sa.umich.edu/ llouder/

Proposed: Benson Farb

Seconded: David Gabai, Walter Neumann

Received: 21 March 2010

Revised: 9 October 2011 